\title{
EFFECT OF PRESTRESS ON THE MECHANICAL PERFORMANCE OF COMPOSITES
}

\author{
A Dissertation \\ by \\ HUANLIN ZHU \\ Submitted to the Office of Graduate and Professional Studies of \\ Texas A\&M University \\ in partial fulfillment of the requirements for the degree of \\ DOCTOR OF PHILOSOPHY
}

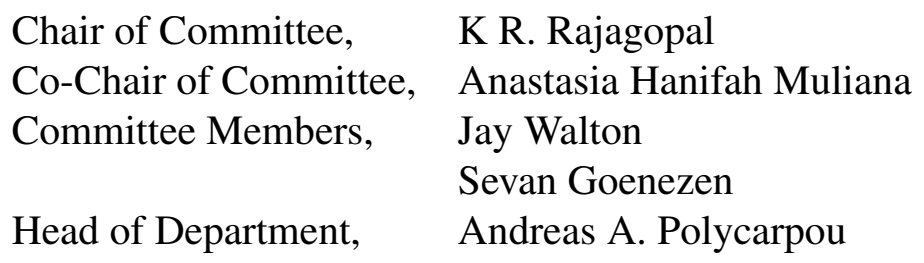

December 2015

Major Subject: Mechanical Engineering

Copyright 2015 Huanlin Zhu 


\begin{abstract}
Bodies are prestressed with the intention to enhance their load carrying capacity. The primary objective of this study is to understand the effect of prestressing the constituents in composite bodies on the overall mechanical performance of the composites. This study considers composites having linearized viscoelastic constituents which can exhibit either fluid-like or solid-like behavior. A new class of elastic and viscoelastic constitutive models with limiting strain or stress softening behavior has also been implemented. The effect of stress relaxation in constituents of the prestressed composite on the overall load carrying capacity of the composite is investigated. In both models discussed above, we deal with linearized strains. Both analytical and numerical results are presented. The properties of the composite, whether a brittle inclusion embedded in ductile matrix or a ductile inclusion in brittle matrix are greatly influenced by the ratio of the induced prestress with respect to the external load and thereby influences the load carrying capacity of the composite.
\end{abstract}




\section{DEDICATION}

To my parents and my husband, for never ceasing in their love, encouragement, and support as I have grown through my life. 


\section{ACKNOWLEDGEMENTS}

I would like to thank my parents for their endless and unconditional support during my entire education. I would like to thank my husband, Huihua for his support and love during the years. I would not have been able to complete this dissertation without their love and encouragement.

I would like to express deepest gratitude to my advisors, Dr. Kumbakonam Rajagopal and Dr. Anastasia Muliana for their guidance and support throughout my research work. Their enthusiasm for the science and engineering inspire me a lot. Their support, expert guidance and encouragement lead me to accomplish this much. I would also extend sincere

gratitude to my advisory committee, Dr. Walton and Dr. Goenezen. Their thoughtful questions and comments inspire my research and lead me through the puzzles. 


\section{NOMENCLATURE}

Note that scalars are represented in plain italics, while bold-faced characters represent either vectors or matrices.

$\mathbb{R} \quad$ Set of all real scalars

$\mathbb{R}^{a \times b} \quad$ Set of all real matrices of size $a \times b$

$\mathbf{A}_{a \times b} \quad$ Matrix in $\mathbb{R}^{a \times b}$

$A_{i j} \quad$ Element in row i of column $\mathrm{j}$ of matrix $\mathbf{A}$

$\mathbf{I}_{n \times n} \quad$ Identity matrix in $\mathbb{R}^{n \times n}$

n Degree of freedoms

* Convolution integral 


\section{TABLE OF CONTENTS}

Page

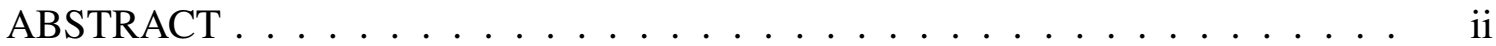

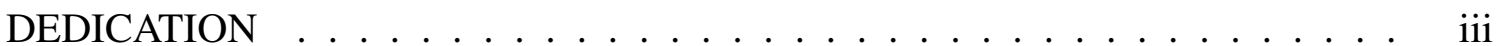

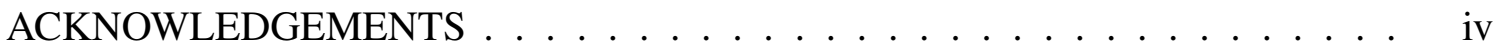

NOMENCLATURE ......................... . .

TABLE OF CONTENTS . . . . . . . . . . . . . . . . . . . . vi

LIST OF FIGURES . . . . . . . . . . . . . . . . . . . . viii

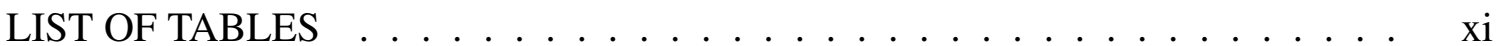

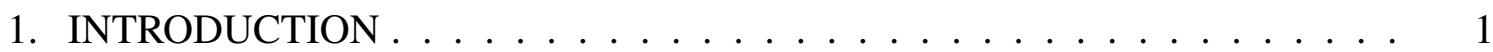

1.1 Literature review . . . . . . . . . . . . . . . . . . . . 2

1.2 Motivation and research objectives .............. 5

2. ANALYTICAL SOLUTIONS OF PRE-STRESSING COMPOSITES . . . . 8

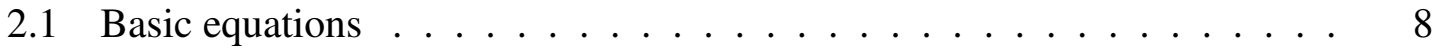

2.1.1 Kinematics ................... 8

2.1.2 Constitutive equations for linearized elastic/viscoelastic model . . 9

2.1.3 Boundary value problem . . . . . . . . . . . . . 10

2.2 Analytical solutions . . . . . . . . . . . . . . . . . . 11

2.2.1 Response of composites with the Maxwell(MX) and SLS models for the matrix . . . . . . . . . . . . . . . 15

2.2.2 The effects of prestresses and instantaneous (elastic) modulus of the matrix . . . . . . . . . . . . . . 18

2.2.3 Response of the composite due to uniaxial tensile load . . . . . 20

2.2.4 Non-uniform prestress in the case of uniaxial loading . . . . . . . 24

2.2.5 Prestressing ductile inclusion in a brittle matrix . . . . . . . 26

3. STUDIES OF INTERFACE/INTERSTITIAL MATTER: FE ANALYSIS . . . . 29

3.1 Analytical/Numerical solutions . . . . . . . . . . . . . . . . 29

3.2 Finite element analysis . . . . . . . . . . . . . . . . . 31 
3.3 Constitutive model . . . . . . . . . . . . . . . . 35

3.4 The effect of particle volume contents and properties of the interstitial matters . . . . . . . . . . . . . . . . . 37

3.5 Prestressing particles through thermo-mechanical process . . . . . . . 40

4. A NEW CLASS OF ELASTIC AND VISCOELASTIC BODIES . . . . . . . . 42

4.1 Nonlinear elastic model . . . . . . . . . . . . . . . . . . . . 42

4.1 .1 Numerical implementation . . . . . . . . . . . . . . . . . 45

4.1.2 Boundary value problem studies . . . . . . . . . . . 46

4.2 Nonlinear viscoelastic model . . . . . . . . . . . . . . . . . . . 52

4.2.1 Numerical implementation . . . . . . . . . . . . . . . . 55

4.2.2 Boundary value problem studies $\ldots \ldots \ldots \ldots 7$

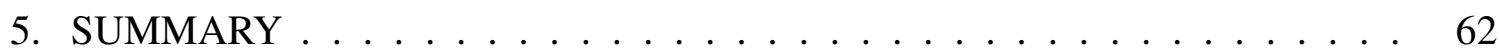

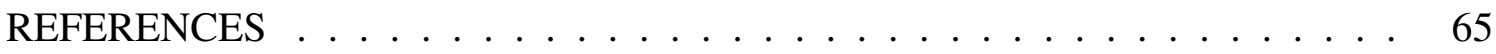

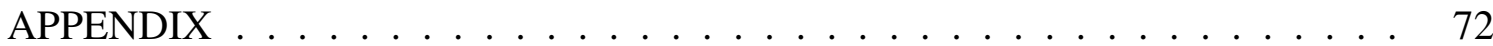




\section{LIST OF FIGURES}

FIGURE

Page

2.1 Hoop stresses along the radial direction at several instants of time [1]. . . 17

2.2 Radial stresses along the radial direction at several instants of time [1]. $\quad 18$

2.3 Hoop stress with and without pre-stressing the inclusion [1]. . . . . . . . 19

2.4 Hoop stress jump at the interface with external tension $T=200 \mathrm{MPa}$ and various prestresses [1]. . . . . . . . . . . . . . . 21

2.5 Stresses with external tensile load $T=200 \mathrm{MPa}$ and internal compressive prestress $P_{0}=200 \mathrm{MPa}$ for $2 \mathrm{D}$ case. The Material parameters are $E^{i}=$ $200 \mathrm{GPa}, \nu^{i}=0.25, \nu_{2}=0.33, \mu_{2}=69 \mathrm{GPa} \cdot \mathrm{s}[1] \ldots \ldots . . . .$.

2.6 Maximum principal stresses in the inclusion at a) $r=0.5 a$ and b) $r=a[1]$. 23

2.7 Maximum shear stress a) in the inclusion $r=0.5 a$ and $b$ ) in the matrix $r=1.5 a[1] \ldots \ldots \ldots \ldots \ldots$

2.8 Hoop stress discontinuities at the interface a) at $\theta=0$ and b) at $t=10 \mathrm{~s}$ [1]. 24

2.9 Maximum principal stress in the inclusion at $t=10 \mathrm{~s}$ varying with respect to angle $\theta$. (a) at $r=0.5 a$, (b) at $r=a[1] \ldots \ldots . . . \ldots$.

2.10 Maximum principal stress in the matrix at $t=10 \mathrm{~s}$ varying with respect to

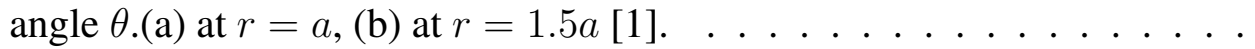

2.11 Maximum shear stress. (a) at the inclusion location $r=0.5 a$, (b) at the matrix location $r=1.5 a[1] . \ldots \ldots 26$

2.12 Hoop stress jump at the interface $r=a$. (a) at $\theta=0$, (b) at $t=10 \mathrm{~s}[1] . \quad$. 26

2.13 Stress fields in the inclusion and matrix at various values of prestresses [1]. 28

2.14 Hoop stress discontinuities at the interface with different values of prestress $[1] . \ldots \ldots \ldots \ldots \ldots$

3.1 Stress field with various matrix dimension in 2D. Dimension of the matrix from left to right: $4 \mathrm{~mm}, 6 \mathrm{~mm}, 10 \mathrm{~mm} \ldots \ldots \ldots$. . . . . . 
3.2 Stress field with various matrix dimension in 3D. Dimension of the matrix from left to right: $4 \mathrm{~mm}, 10 \mathrm{~mm} . \ldots \ldots \ldots$

3.3 Composite model with various particle volume fractions. . . . . . . . 33

3.4 Mesh convergence study. . . . . . . . . . . . . . . . 34

3.5 The effect of particle volume contents on the overall response of compos-

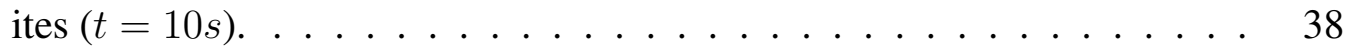

3.6 The effect of stiff and soft interphases on the overall response of composites with $10 \%$ particle volume content $(t=10 s)$. Top: inclusions; middle: interstitial matters; bottom: matrix. . . . . . . . . . . . . 39

3.7 Maximum principal strain contours in composites with $10 \%$ particle volume content at early and later times. Top: inclusions; bottom: matrix. . . 39

3.8 Maximum principal stresses in the constituents due to a temperature change. 41

3.9 Maximum principal stress with and without thermal process. . . . . . . 41

4.1 Uniaxial response for one element with various models in comparison with linear elastic model. . . . . . . . . . . . . . . 46

4.2 Hoop stresses and strains for composites with one stiffer inclusion. . . . 47

4.3 Hoop stresses and strains for composite with a softer inclusion. . . . . . 48

4.4 Hoop stresses with and without prestress for various nonlinear elastic models in comparison with linear elastic model. . . . . . . . . . . . . . 48

4.5 Hoop strains with and without prestress for various nonlinear elastic models in comparison with linear elastic model. . . . . . . . . . . . . . 49

4.6 Maximum principal stress and strain with and without prestress. . . . . 51

4.7 Uniaxial response for one element for nonlinear viscoelastic models in comparison with linear viscoelastic model. . . . . . . . . . . . . 57

4.8 Hoop strain dependence on time for nonlinear viscoelastic model showing stress softening behavior. . . . . . . . . . . . . 58

4.9 Hoop strain dependence on time for nonlinear viscoelastic model showing limiting strain behavior. . . . . . . . . . . . . . 59

4.10 Maximum principal stress and strain with and without prestress. . . . . 60 
4.11 Maximum principal strain dependence on time with matrix showing stress softening behavior. . . . . . . . . . . . . . . 60

4.12 Maximum principal strain dependence on time with matrix showing limiting strain behavior. ................. 61

4.13 Axial displacement for three viscoelastic models. . . . . . . . . . . . . 61 


\section{LIST OF TABLES}

TABLE $\quad$ Page

2.1 Properties of the constituents $[1] \ldots \ldots \ldots \ldots$

3.1 Elastic properties and Prony series coefficients for the matrix [2]. . . . . 34

3.2 Linear thermo-elastic properties of constituents. . . . . . . . . 35

4.1 Nonlinear elastic material parameters [3]. . . . . . . . . . . . . . 44

4.2 Elastic parameters for inclusion. . . . . . . . . . . . . . . 47

4.3 Time dependent functions [3]. . . . . . . . . . . . . . . . . . . . . . 54

4.4 Recursive-iterative algorithm. . . . . . . . . . . . . 56 


\section{INTRODUCTION ${ }^{*}$}

Composite materials are appealing in many structural applications since they can be customized and engineered to achieve desired performances by varying constituent properties and microstructural arrangements. Despite their promising advantages, there are challenging scientific issues that continue to face engineers in designing and using composites. The multi-component and multi-scale nature of composites presents heterogeneities at various scales: laminate, ply, and constituents, which cause stress discontinuities at the interface between the layers and constituents, leading to debonding. When debonding or delamination occurs, it disrupts load transfers between constituents, reducing the overall performance of composites and shortening the life of composites. An effort has been made to improve bonding performance between constituents by the development of new adhesives and/or surface treatments of the inclusions: fibers or particles. Unfortunately, a significantly strong bonding at the interface could lead to fracture or cracking in the fiber and/or matrix constituents, especially when brittle constituents are considered. Alternatively, prestressing method has been used to improve load carrying capacity of the composites. We feel that the pre-stressing (especially compressive stresses) of one or more of the constituents of a composite body will lead to enhancing the load-carrying capacity of the composite. The ultimate goal is to carry out a novel theoretical and computational study that assesses the overall performance of composites due to pre-stressing, at the microscopic level, one or more of the components of a composite with a view towards the design and development of such bodies.

"Portions of this section have been reprinted with permission from ASCE from "Effect of prestress on the mechanical performance of composites” by Zhu, H., Muliana, A., and Rajagopal, K, 2015. J. Eng. Mech., 141(7), 04015011. 


\subsection{Literature review}

The idea of prestressing, which refers to inducing internal stresses to improve the external load carrying capacity of structures, has been considered for decades, mainly with regard to large scale infrastructures such as bridges, pipes, aircraft fuselages, etc. [4, 5, 6]. In reinforced concrete structures, the steel reinforcement is pre-stressed so that the overall reinforced concrete structures are under compression, increasing the tension load carrying capacity of the structures. Pre-stresses in civil structures have also been considered by constructing curved structures. In manufacturing metallic pipes heating and cooling processes are applied, a shrink-fit problem, to create residual stresses in the pipes. Several experimental and analytical investigations have been conducted on prestressed steelconcrete composite (PSCC) beams [7, 8, 9, 10, 11]. On the basis of these experiments, it is suggested that compared to the conventional concrete beams, PSCC beams have several advantages such as extending the elastic range, increasing the load carrying capacity, decreasing the structural weight and improving the fatigue and fracture resistance $[10,12]$.

Except for the commonly used prestressing technique of structural materials like concrete, research in applying prestressing technique with regard to other systems such as fiber-reinforced plastic (FRP) composites are relatively recent. FRP composites are widely used in construction applications [13]. Mechanical testing of prestressed FRP composites shows that the compressive prestress which is induced by fiber tension during curing can significantly increase impact resistance, tensile strength, elastic modulus and flexural properties of the composites $[14,15]$. The pre-stress has been applied to fiber reinforced polymer (FRP) laminated composites cylinders under hydrostatic pressure like in submarine applications, in which a ply is pre-stressed by applying tension in order to minimize axial and hoop stresses [16]. At a smaller scale, i.e., microstructural scale, fiber pre-stress has also been considered in manufacturing fiber reinforced composites in order to minimize 
fiber waviness and overcome thermal residual stresses due to the processing at elevated temperatures $[16,17,18]$. In a FRP composite, comprising of nylon fibers and polyester resin, pre-stressing the fiber could significantly improve the matrix fracture toughness and increase the energy absorber of the composite by $25 \%$ [19].

While most of the research that has been carried out concerns the development of various prestressing techniques such as induced prestress of elastic and viscoelastic bodies [19] and experimental testing characterizing the mechanical properties of composites with prestress [20], few systematic analytical studies have been carried out from which one can get a good understanding of the effect of prestressing the constituents on the overall mechanical performance of composite materials. Studying the effect of prestress by considering detailed constitutive models of the constituents, i.e., models to describe elastic-plastic, viscoelastic, visco-plastic, and other such models, has rarely been done before. The effect of pre-stressing on constituents that are microscopic in size and then assessing the change in response of the macroscopic composite due to the pre-stresses at the microscopic level has not been studied.

Polymers have been used widely in replacement of many conventional materials. The advantages of polymers are low cost and easy processing. However, they have some limitations such as low stiffness and low strength [21]. To extend their applications, polymers are often reinforced with particulate fillers, such as glass, micro-/nano- $\mathrm{SiO}_{2}$, carbon nanotubes to improve their physical and mechanical properties. The particulate reinforced polymer composites have been investigated for their significant improvement in mechanical properties $[22,23,24,25]$. Extensive studies on the dependence of the mechanical properties on the particle sizes have been conducted [26, 27]. The effect of prestressing in the inclusion and particle-matrix interface adhesion condition have been overlooking. The manufacturing processes and techniques for processing composites are, however, not covered here. 
Furthermore, it has been shown recently that the class of elastic bodies is wider than the classical Cauchy and Green elastic bodies [28, 29, 30, 31], if by an elastic body one means a body incapable of dissipation. Later, a new class of quasi-linear model is formulated to describe the nonlinear viscoelastic response of materials [32]. This study considers a specific model that belongs to the general class introduced by Rajagopal [33]. This limiting small strain model can describe the behavior of certain polymeric solids that exhibit limited stretch. This limiting strain model is not the only model that can describe the limited strain behaviour of materials. There are research concerning the description of the phenomenon of limited chain extensibility using elastic models [34]. However, this newly proposed model presents another interesting way to describe materials and solving boundary value problems where classical linearized elastic model cannot succeed. There are already some interesting results provided by Rajagopal and co-workers [28, 29, 30, 31, 33] by applying this new model to solve some simple boundary value problems.

The correspondence principle between elastic solids and linear viscoelastic materials is considered for solving the overall deformation of composites having viscoelastic constituents. The correspondence principle for obtaining solutions to problems in linear viscoelasticity from the solution to the corresponding problem in elasticity is well established [35]. Rajagopal and Wineman [36] extended the correspondence principle to the case of a material defined through a linear constitutive relation that satisfy certain conditions and thus the work in this study can be extended to other linear materials. Recently, Rajagopal and Wineman [37] extended the correspondence to that between elastic and quasi-linear viscoelastic materials, when certain conditions are met. Thus, one can also extend the results to the case of quasilinear viscoelastic materials, a class that is used widely to describe biological solids.

Numerical algorithms that are compatible with general displacement based FE structural analyses for uncoupled thermo-mechanical analysis have been developed mainly for 
analyzing viscoelastic constitutive model and the limiting small strain elastic/viscoelastic constitutive models. The numerical algorithm will be called at every material (Gaussian) integration point within finite elements at each structural iteration to achieve structural and material convergences simultaneously. For visoelastic models, there are many types of algorithm such like incremental algorithm [38,39] where the strain rate was modeled as functions of the current stresses and the accumulated strains. The recurrence numerical algorithm introduced by Taylor et al. [40] has also been used for solving a linear viscoelastic integral equation. This approach has been used and developed to incorporated nonlinear effects due to stress, temperature, moisture, and aging [41, 42, 2, 43]. The method bypasses the need to store entire history variables, which minimizes the storage required in performing the integration. The convolution integral is divided into recursive parts and allows the incremental formulation and integration for the current stress state from the history variables stored at the previous time step, and the current time and strain increments. For the implementation of the new class of quasilinear viscoelastic model, this study adopts the one dimension algorithm reported in Muliana et al. [32] and extends it to three dimensional problems.

\subsection{Motivation and research objectives}

Understanding the effect of prestressing the constituents on the overall mechanical performance of composite materials has rarely been carried out. This work is aimed at understanding the effect of pre-stressing the constituents of composites with a view towards enhancing the overall load carrying capacity of the composites and studying the effect of microstructural morphology on the overall mechanical performance of composites through the use of proper constitutive models for each constituent. This study starts with simplified microstructures of composites, i.e., a circular inclusion embedded in twodimensional (2D) infinite matrix domain and a solid spherical inclusion placed in three- 
dimensional (3D) infinite matrix. Exact analytical solutions based on linearized elasticity and linearized viscoelasticity are obtained to understand the effects of prestressing and properties of the constituents on the overall performance of the composites. The main purpose of considering the simplified microstructures is to understand the feasibility in enhancing the load carrying capacity of composites by prestressing the constituents. The next step considers more realistic composite microstructures having several solid spherical particle arrangements dispersed in homogeneous matrix. Finite element (FE) method is used to obtain approximate solutions to the boundary value problems. The overall performance of composites with different interface/interphase behaviors and prestressed inclusions is examined. A limiting small strain constitutive model which belongs to a new class of elastic/visoelastic bodies is implemented for the matrix constituent. The comparison with the classical elastic/visoelastic constitutive model is discussed. More realistic constitutive models that include time-dependence and a new class of elastic/viscoelastic model are incorporated. The ultimate goal is to carry out a novel theoretical and computational study that assesses the overall performance of composites due to pre-stressing, at the microscopic level, one or more of the components of a composite with a view towards the design and development of such bodies.

The objectives of the current study are:

1) Analyze the overall performance of two composite systems comprising of a) brittle inclusions (glass, ceramics) in a ductile matrix (metals, polymers) and b) ductile inclusions in a brittle matrix. Linearized elastic response is considered for the brittle constituent while linearized viscoelastic constitutive model is used for the polymeric constituent. Both twodimensional (2D) composites, having a circular inclusion, and three-dimensional (3D) composites with a spherical inclusion are studied.

2) Perform numerical (finite element) analysis to examine the effects of different microstructural arrangements and residual (pre-) stresses on the overall mechanical perfor- 
mance of the composites. Composites with various interface matter and various volume fractions are considered.

3) Formulate nonlinear limiting small strain and stress softening constitutive models which belong to the new class of elastic/viscoelastic bodies and discuss the results in contrast to the classical linearized elastic/viscoelastic bodies. Analyze the effect of the presence of the inhomogeneity on the field variables of the body.

4) Study the effect of thermo-mechanical cycles during processing on the overall properties of the composites. Discuss a possible method to induce prestress on the brittle inclusions, coated by soft polymer interphases, by temperature changes. 


\section{ANALYTICAL SOLUTIONS OF PRE-STRESSING COMPOSITES*}

This sections starts with briefly presenting kinematics and constitutive models with linearized elastic and viscoelastic constituents. Analytical solutions for a single inclusion embedded in a infinite medium for both $2 \mathrm{D}$ and 3D cases are discussed. The main purpose of considering the simplified microstructures is to understand the feasibility in enhancing the load carrying capacity of composites by prestressing the constituents.

\subsection{Basic equations}

\subsubsection{Kinematics}

Let $\mathrm{X} \in \boldsymbol{\kappa}_{R}(\mathcal{B})$ denote a particle belonging to a body $\mathcal{B}$ in the reference configuration $\kappa_{R}(\mathcal{B})$, and $\mathbf{x} \in \boldsymbol{\kappa}_{t}(\mathcal{B})$ denote the position of the same particle in the current configuration $\kappa_{t}(\mathcal{B})$, at time t. The mapping $\chi$ assigns each particle $\mathbf{X} \in \kappa_{R}(\mathcal{B})$ the position $\mathbf{x}$ at time $\mathrm{t}$, that is, $\mathbf{x}=\chi(\mathbf{X}, t)$. We assume $\chi$ is sufficiently smooth to make all the derivatives that are taken to be meaningful. Let us denote the displacement $\mathbf{u}$, the deformation gradient $\mathbf{F}$ through

$$
\mathbf{u}:=\mathbf{x}-\mathbf{X}, \quad \mathbf{F}:=\frac{\partial \boldsymbol{\chi}}{\partial \mathbf{X}}
$$

We define the Cauchy-Green stretch tensor B and $\mathbf{C}$ through

$$
\mathbf{B}:=\mathbf{F F}^{T}, \quad \mathbf{C}:=\mathbf{F}^{T} \mathbf{F}
$$

and Green-St.Venant strain E through

$$
\mathbf{E}:=\frac{1}{2}(\mathbf{C}-\mathbf{I})
$$

"Portions of this section have been reprinted with permission from ASCE from "Effect of prestress on the mechanical performance of composites” by Zhu, H., Muliana, A., and Rajagopal, K, 2015. J. Eng. Mech., 141(7), 04015011. 
Under the assumption

$$
\max _{\mathbf{x} \in \kappa(B), t \in \mathbb{R}}\left\|\frac{\partial \mathbf{u}}{\partial \mathbf{x}}\right\|=0(\delta), \delta \ll 1
$$

where $\|$.$\| stands for the usual trace norm. We have the linearized strain \varepsilon$

$$
\left.\boldsymbol{\varepsilon}=\frac{1}{2}\left[\left(\frac{\partial \mathbf{u}}{\partial \mathbf{x}}\right)+\frac{\partial \mathbf{u}}{\partial \mathbf{x}}\right)^{T}\right]
$$

\subsubsection{Constitutive equations for linearized elastic/viscoelastic model}

The constitutive equation for a classical linearized elastic material is:

$$
\boldsymbol{\varepsilon}=\frac{1+\nu}{E} \boldsymbol{\sigma}-\frac{\nu}{E}(\operatorname{tr} \boldsymbol{\sigma}) \mathbf{I}
$$

The Cauchy stress $\boldsymbol{\sigma}$ in a generalized linearized viscoelastic model is given as:

$$
\boldsymbol{\sigma}=2 \mu_{R} * d \boldsymbol{\varepsilon}+\left(K_{R}-\frac{2}{3} \mu_{R}\right) * d(\operatorname{tr} \boldsymbol{\varepsilon}) \mathbf{I}
$$

where $\mu_{R}, K_{R}$ are the shear relaxation modulus and bulk relaxation modulus, respectively. $\varepsilon$ is the linearized strain. $*$ denotes the convolution integral.

The model can also be expressed equivalently by defining the linearized strain as:

$$
\boldsymbol{\varepsilon}=(1+\pi) * \mathrm{~d} J * \mathrm{~d} \boldsymbol{\sigma}-\pi * \mathrm{~d} J * \mathrm{~d}(\operatorname{tr} \boldsymbol{\sigma}) \mathbf{I}
$$

where we refer $\pi$ as the normal strain contraction ratio function, sometime referred as Poisson's effect and $J$ as the creep compliance. This former notion is not strictly correct as the response of a viscoelastic fluid depends on the strain rate and one does not have a stress-strain relation for such materials and this material function is strain rate dependent. 
However, for slow enough "flows" we shall assume that this function is in fact a constant.

Polymers in general can have time-dependent Poisson's effect. However, time-independent Poisson's effect for polymeric materials has also been reported. For example, Schapery mentions that Poisson's function of epoxy is very nearly constant even when the uniaxial and shear moduli are significantly time- and frequency-dependent [44]. Moal and Perreus experimentally found that the Poisson's function of a tested resin is invariant with respect to time [45]. This study chooses Poisson's effect to be a constant, however, it is straightforward to allow Poisson's function to be time dependent.

\subsubsection{Boundary value problem}

The boundary value problem are solved by satisfying the following conditions:

$$
\begin{array}{rlrl}
\operatorname{div} \boldsymbol{\sigma}=\mathbf{0} & \mathbf{x} \in \kappa_{t}(\mathcal{B}) \\
\boldsymbol{\sigma} \mathbf{n}=\hat{\mathbf{t}} \quad \mathbf{x} \in \partial \boldsymbol{\kappa}_{t}^{t}(\mathcal{B}), \quad \mathbf{u}=\hat{\mathbf{u}} \quad \mathbf{x} \in \partial \boldsymbol{\kappa}_{t}^{u}(\mathcal{B})
\end{array}
$$

where $\hat{\mathbf{t}}$ and $\hat{\mathbf{u}}$ are the prescribed traction and displacement on the boundaries $\partial \boldsymbol{\kappa}_{t}^{t}(\mathcal{B})$, $\partial \boldsymbol{\kappa}_{t}^{u}(\mathcal{B})$, respectively. And $\partial \boldsymbol{\kappa}_{t}^{t}(\mathcal{B}) \cup \partial \boldsymbol{\kappa}_{t}^{u}(\mathcal{B})=\partial \boldsymbol{\kappa}_{t}(\mathcal{B}), \partial \boldsymbol{\kappa}_{t}^{t}(\mathcal{B}) \cap \partial \boldsymbol{\kappa}_{t}^{u}(\mathcal{B})=\varnothing$. 


\subsection{Analytical solutions}

This study starts with simplified microstructures of composites and exact analytical solutions based on linearize elasticity and linearized viscoelasticity are obtained to understand the effects of prestressing and properties of the constituents on the overall performance of composites. The main purpose of considering the simplified microstructures is to understand the feasibility in enhancing the load carrying capacity of composites by prestressing the constituents. Also, the comparison of results from exact solutions and numerical solutions justfy the use of numerical approximation.

Consider a 2D composite, comprising of a solid circular inclusion, or a 3D composite, having a solid spherical inclusion, of a radius $a$, embedded in a matrix of infinite medium with a uniform constant traction $T$ applied at the boundary. The first composite system under consideration comprises of a brittle inclusion bonded to a ductile matrix. There are many examples of composite materials that belong to this category, such as glassy/carbon inclusion in polymeric matrix, ceramic inclusion in metallic/polymeric matrix, brittle aggregate in asphalt matrix, etc.

It is assumed that the inclusion behaves like linear elastic isotropic and homogeneous solids, while the matrix is comprised of a linear viscoelastic body. Both the Linear Maxwell fluid model and the Standard linear solid (SLS) model are employed to describe the linear viscoelastic behavior of the matrix. It is further assumed that the inclusion and the matrix are perfectly bonded.

The solutions to field variables, i.e., deformation and stress fields in the inclusion and matrix, are determined by imposing equilibrium and compatibility conditions and prescribing boundary conditions. We appeal to the correspondence principle between the linear elastic solids and the linear viscoelastic materials for solving the overall deformation of composites having viscoelastic constituents. The normal stresses and displacement in the 
2D case, which vary with radial location $r$, and time $t$, are summarized as:

$$
\begin{aligned}
\sigma_{r r}^{i}(r, t) & =\sigma_{\theta \theta}^{i}(r, t)=P(t)-P_{0} \\
\sigma_{r r}^{m}(r, t) & =T\left(1-\frac{a^{2}}{r^{2}}\right)+\frac{a^{2}}{r^{2}} P(t) \\
\sigma_{\theta \theta}^{m}(r, t) & =T\left(1+\frac{a^{2}}{r^{2}}\right)-\frac{a^{2}}{r^{2}} P(t) \\
u_{r}^{i}(r, t) & =\frac{r}{E^{i}}\left(\sigma_{\theta \theta}^{i}(r, t)-\nu^{i} \sigma_{r r}^{i}(r, t)\right) \\
u_{r}^{m}(r, t) & =r\left[J^{m}(t)\left(2 T-P(0)\left(1+\pi^{m}\right)\right)\right. \\
& \left.\left.-\int_{0}^{t}\left(1+\pi^{m}\right) J^{m}(t-s) \frac{\mathrm{d} P(s)}{\mathrm{d} s} \mathrm{~d} s\right)\right]
\end{aligned}
$$

where the superscripts $i$ and $m$ denote the inclusion and matrix, respectively, the subscript $r r$ or $r$ and $\theta \theta$ indicate the radial and circumferential (hoop) components, respectively. $E^{i}$ and $\nu^{i}$ are the elastic modulus and Poisson's ratio for the elastic inclusion, respectively, while $J^{m}(t)$ and $\pi^{m}$ are the time-dependent compliance and the contracting ratio for the viscoelastic matrix. The pre-existing compressive prestress in the inclusion is $P_{0}$ and the radial stress at the interface is $P(t)$, which varies with time due to the viscoelastic response of the matrix. For the problem under consideration, the shear components of stresses and strains are absent. For the viscoelastic matrix, we considered two models: Maxwell fluid model and Standard linear solid model. Maxwell fluid model is chosen for the viscoelastic fluid-like matrix, while the Standard Linear Solid model is used for the viscoelastic solidlike matrix.

For the linearized Maxwell model, the creep compliance is:

$$
J(t)=\frac{1}{E}+\frac{t}{\mu},
$$

where $E$ is the elastic constant of the spring and $\mu$ is the viscosity of the dash-pot. 
For the SLS model, the creep compliance is:

$$
J(t)=\frac{1}{E}-\frac{E_{0}}{E\left(E+E_{0}\right)} e^{-\frac{E E_{0}}{\mu\left(E+E_{0}\right)} t}
$$

where $E$ and $E_{0}$ are the elastic constant of the springs and $\mu$ is the viscosity of the dash-pot.

As a result, the time-dependent radial stress at the interface, $P(t)$, when the Maxwell model is considered for the matrix is:

$$
\begin{aligned}
P(t)= & \frac{E\left(1-\nu^{i}\right)\left(P_{0}\left(1+\pi^{m}\right)-2 T\right)}{\left(E\left(1-\nu^{i}\right)+E^{i}\left(1+\pi^{m}\right)\right)\left(1+\pi^{m}\right)} \\
& \exp \left(-\frac{E^{i} E\left(1+\pi^{m}\right)}{\mu\left(E\left(1-\nu^{i}\right)+E^{i}\left(1+\pi^{m}\right)\right)} t\right)+\frac{2}{1+\pi^{m}} T
\end{aligned}
$$

and when the SLS model is considered:

$$
\begin{aligned}
P(t) & =\frac{E_{0} E^{i}\left(1-\nu^{i}\right)\left(P_{0}\left(1+\pi^{m}\right)-2 T\right)}{\left(E\left(1-\nu^{i}\right)+E^{i}\left(1+\pi^{m}\right)\right)\left(\left(E+E_{0}\right)\left(1-\nu^{i}\right)+E^{i}\left(1+\pi^{m}\right)\right)} \\
& \exp \left(-\frac{E_{0}\left(E\left(1-\nu^{i}\right)+E^{i}\left(1+\pi^{m}\right)\right)}{\mu\left(\left(E+E_{0}\right)\left(1-\nu^{i}\right)+E^{i}\left(1+\pi^{m}\right)\right)} t\right) \\
& -\frac{2 E^{i}\left(1-\pi^{m}\right)}{E\left(1-\nu^{i}\right)+E^{i}\left(1+\pi^{m}\right)} T \\
& +\frac{E\left(1-\nu^{i}\right)}{E\left(1-\nu^{i}\right)+E^{i}\left(1+\pi^{m}\right)} P_{0}
\end{aligned}
$$

The stress and displacement fields can be determined by substituting $P(t)$ from either Eq. 2.17 or Eq. 2.18 into Eqs. 2.10-2.14.

In a similar manner, the response of the composite in $3 \mathrm{D}$ domain can be obtained. Like in the $2 \mathrm{D}$ problem, it is assumed that the inclusion behaves like linear elastic isotropic and homogeneous solids, while the matrix is comprised of a linear viscoelastic body and that the inclusion and the matrix are perfectly bonded. The same variables and material properties used in the 2D case are considered for the 3D composites. The field variables 
are listed as following:

$$
\begin{aligned}
\sigma_{r r}^{i}(r, t) & =\sigma_{\theta \theta}^{i}(r, t)=\sigma_{\varphi \varphi}^{i}(r, t)=P(t)-P_{0} \\
\sigma_{r r}^{m}(r, t) & =T\left(1-\frac{a^{3}}{r^{3}}\right)+\frac{a^{3}}{r^{3}} P(t) \\
\sigma_{\theta \theta}^{m}(r, t) & =\sigma_{\varphi \varphi}^{i}(r, t)=T\left(1+\frac{a^{3}}{2 r^{3}}\right)-\frac{a^{3}}{2 r^{3}} P(t) \\
u_{r}^{i}(r, t) & =\frac{r}{E^{i}}\left(\sigma_{\theta \theta}^{i}(r, t)-\nu^{i} \sigma_{r r}^{i}(r, t)-\nu^{i} \sigma_{\varphi \varphi}^{i}(r, t)\right) \\
u_{r}^{m}(r, t) & =\left(\left((1.5 T-0.5 P(0)) J(t)-0.5 \int_{0}^{t} J(t-s) \frac{\mathrm{d} P(s)}{\mathrm{d} s} \mathrm{~d} s\right)\right. \\
& -\pi^{m}\left(\left(P(0) J(t)+\int_{0}^{t} J(t-s) \frac{\mathrm{d} P(s)}{\mathrm{d} s} \mathrm{~d} s\right)\right. \\
& \left.\left.+\left((1.5 T-0.5 P(0)) J(t)-0.5 \int_{0}^{t} J(t-s) \frac{\mathrm{d} P(s)}{\mathrm{d} s} \mathrm{~d} s\right)\right)\right)
\end{aligned}
$$

where $P(t)$ is the time-dependent radial stress at the interface as in $2 \mathrm{D}$ problem. When the Maxwell model and SLS model are considered for the matrix, $P(t)$ takes the following form respectively:

$$
\begin{aligned}
P(t) & =-\frac{E\left(\left(P_{0}\left(\nu^{i}\left(1+\pi^{m}\right)\right)-0.5\left(1+\pi^{m}\right)\right)+T\left(-3 \nu^{i}\left(1-\pi^{m}\right)-1.5 \pi^{m}+1.5\right)\right)}{0.5\left(1+\pi^{m}\right)\left(0.5 E^{i}\left(1+\pi^{m}\right)-E\left(1-2 \pi^{m}\right)\right)} \\
& \exp \left(-\frac{0.5 E^{i} E\left(1+\pi^{m}\right)}{\mu_{2}\left(E\left(1-2 \nu^{i}\right)+0.5 E^{i}\left(1+\pi^{m}\right)\right)} t\right)+\frac{3\left(1-\pi^{m}\right) T}{1+\pi^{m}} \\
P(t) & =-\frac{E^{i} E_{0}\left(P_{0}\left(-0.5-0.5 \pi^{m}+\nu^{i}\left(1+\pi^{m}\right)\right)+T\left(1.5-1.5 \pi^{m}+\nu^{i}\left(-3+3 \pi^{m}\right)\right)\right)}{\left(0.5 E^{i}\left(1+\pi^{m}\right)+E\left(1-2 \nu^{i}\right)\right)\left(0.5 E^{i}\left(1+\pi^{m}\right)+\left(E+E_{0}\right)\left(1-2 \nu^{i}\right)\right)} \\
& \exp \frac{-E_{0}\left(0.5 E^{i}\left(1+\pi^{m}\right)+E\left(1-2 \nu^{i}\right)\right) t}{\mu\left(0.5 E^{i}\left(1+\pi^{m}\right)+\left(E+E_{0}\right)\left(1-2 \nu^{i}\right)\right)} \\
& -\frac{E P_{0}\left(-1+2 \nu^{i}\right)+E^{i} T\left(-1.5+1.5 \pi^{m}\right)}{0.5 E^{i}\left(1+\pi^{m}\right)+E\left(1-2 \nu^{i}\right)}
\end{aligned}
$$

It is noted that the real composite systems occupy a 3D space, thus a $3 \mathrm{D}$ solution is more realistic than 2D solution. However, 2D solutions are generally simpler and have 
been widely used in obtaining rough estimates of the response of composites prior to designing the composite materials and/or when designing structures made of composites. One of the intentions of this study is to examine the responses from both 2D and 3D cases which provides justification of the use of simpler 2D analysis.

\subsubsection{Response of composites with the Maxwell(MX) and SLS models for the matrix}

Consider a composite with a uniform external tension $T=200 \mathrm{MPa}$ prescribed on the boundary of the infinite matrix and a pre-existing compressive prestress $P_{0}=200 \mathrm{MPa}$ prescribed on the inclusion. The material parameters for the inclusion and viscoelastic matrix, both with Maxwell and SLS models, are presented in Table 2.1.

Table 2.1: Properties of the constituents [1].

\begin{tabular}{|l|c|c|}
\hline Constituents & Parameters & Values \\
\hline Elastic inclusion & $E^{i}$ & $200 \mathrm{GPa}$ \\
& $\nu^{i}$ & 0.25 \\
\hline Maxwell matrix & $E$ & $100 \mathrm{GPa}$ \\
& $\pi^{m}$ & 0.33 \\
& $\mu$ & $69 \mathrm{GPa} \cdot \mathrm{s}$ \\
\hline SLS matrix & $E$ & $50 \mathrm{GPa}$ \\
& $E_{0}$ & $50 \mathrm{GPa}$ \\
& $\pi^{m}$ & 0.33 \\
& $\mu$ & $69 \mathrm{GPa} \cdot \mathrm{s}$ \\
\hline
\end{tabular}

Figures 2.1 and 2.2 depict the circumferential (hoop) and radial stress fields, respectively, in the composites at several instants of time: early time, some time during relax- 
ation, and later time, obtained from 2D and 3D analysis using both the Maxwell and SLS viscoelastic matrix model. It is seen that the differences in the hoop stresses from the 2D and 3D analysis are quite significant, especially for the hoop stress discontinuity at the interface, while the radial stresses from the $2 \mathrm{D}$ and $3 \mathrm{D}$ analysis are sufficiently close. High values in the hoop stress discontinuities can lead to debonding between the inclusion and matrix, reducing the load carrying capacity of the composites. Although 2D analysis are typically less complicated, they might lead to inaccurate prediction of the problems. Especially in this case, if one wants to understand the debonding issues at the interface and enhance the load carrying capacity by avoiding debonding, it is perhaps necessary to consider 3D analysis. Next, it is also observed that for the above composites under external tensile load, the magnitude of the tensile stress in the brittle inclusion and in the matrix decreases significantly by introducing the compressive prestress, as illustrated in Fig. 2.3. Reducing the tensile stresses in the brittle inclusion and ductile matrix can delay or minimize failure in the constituents due to cracking. It is also seen that when the Maxwell fluid model is considered, the matrix undergoes more significant relaxation than when the SLS model is considered, which is expected since the Maxwell fluid model experiences continuous relaxation. 


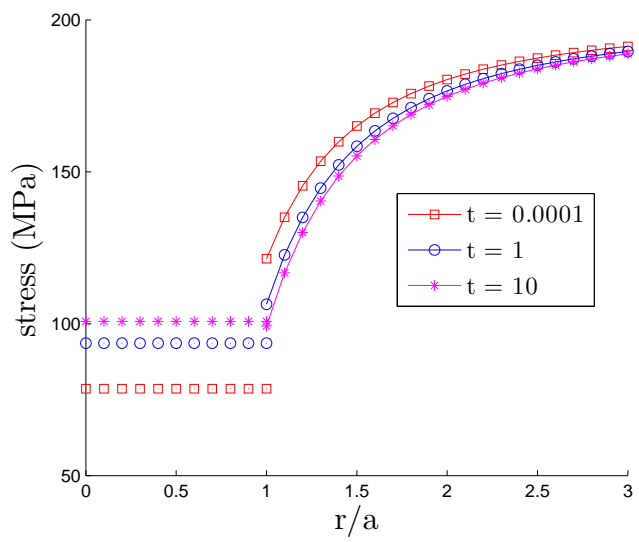

(a) Maxwell model for 2D case.

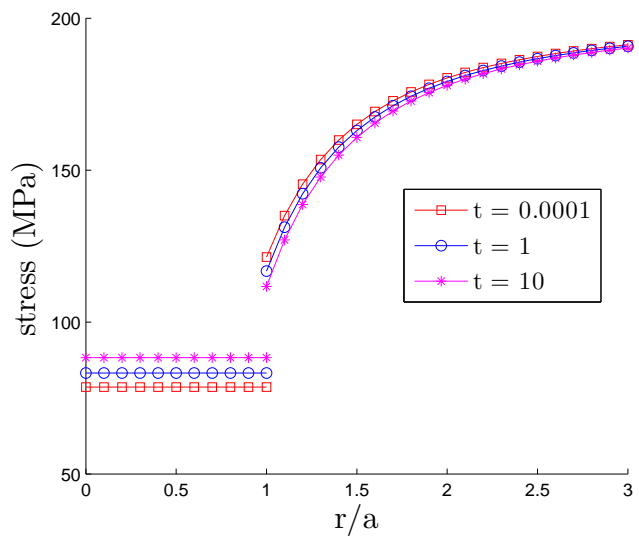

(c) SLS model for 2D case.

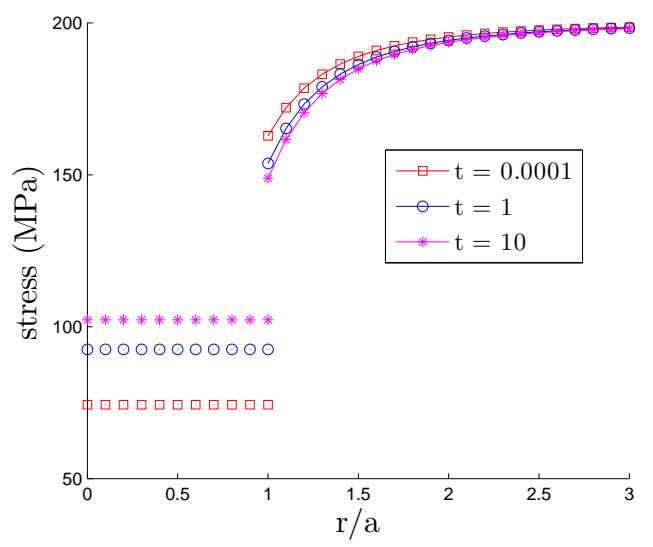

(b) Maxwell model for 3D case.

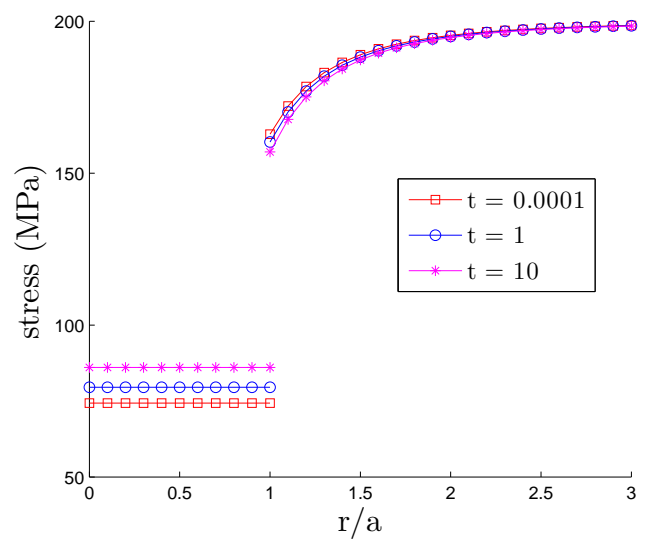

(d) SLS model for 3D case.

Figure 2.1: Hoop stresses along the radial direction at several instants of time [1]. 


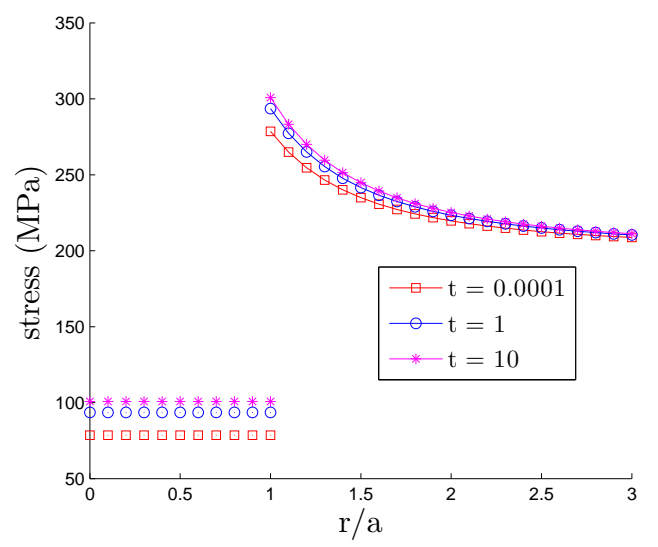

(a) Maxwell model for 2D case.

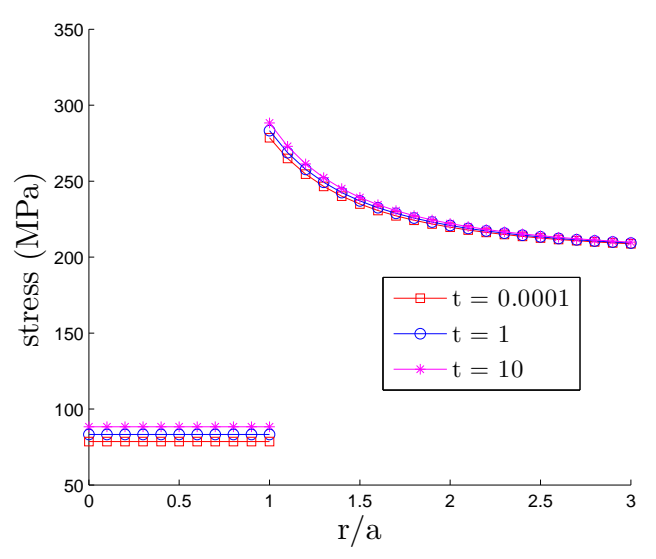

(c) SLS model for 2D case.

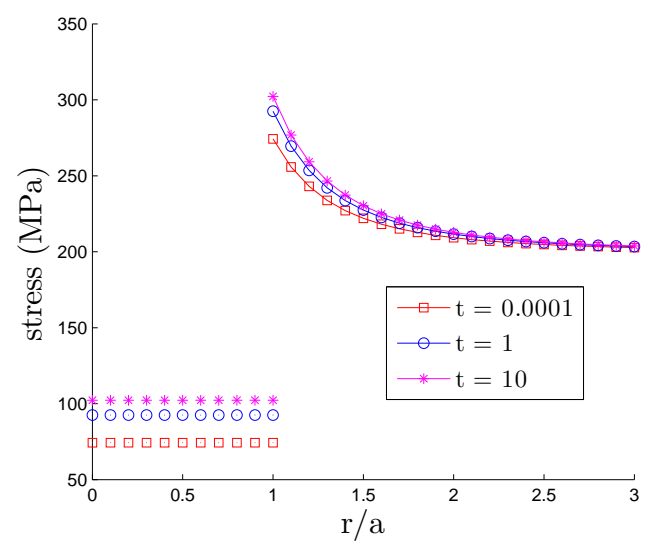

(b) Maxwell model for 3D case.

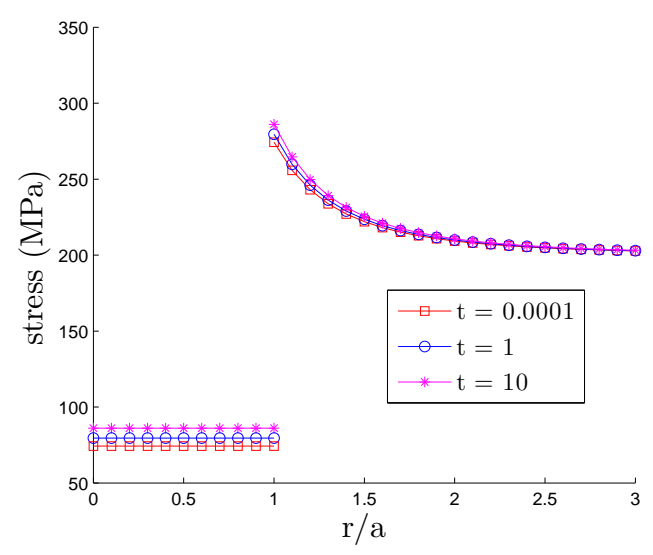

(d) SLS model for 3D case.

Figure 2.2: Radial stresses along the radial direction at several instants of time [1].

\subsubsection{The effects of prestresses and instantaneous (elastic) modulus of the matrix}

As both Maxwell and SLS show similar trends in terms of the stress fields in the composites, in this section the discussion is confined to the Maxwell viscoelastic matrix. An excellent example for a composite comprising of an elastic solid and a viscoelastic fluid is the earth wherein the outer crust is usually approximated as an elastic solid while the mantle is modeled as a viscoelastic fluid. Of course, in the model of the earth we also need to deal with an inner core. However, for certain applications one is mainly concerned 


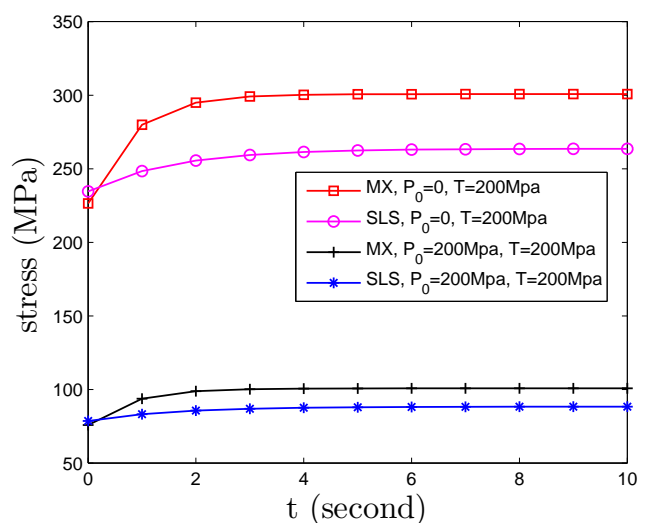

(a) Stresses in the inclusion for 2D case.

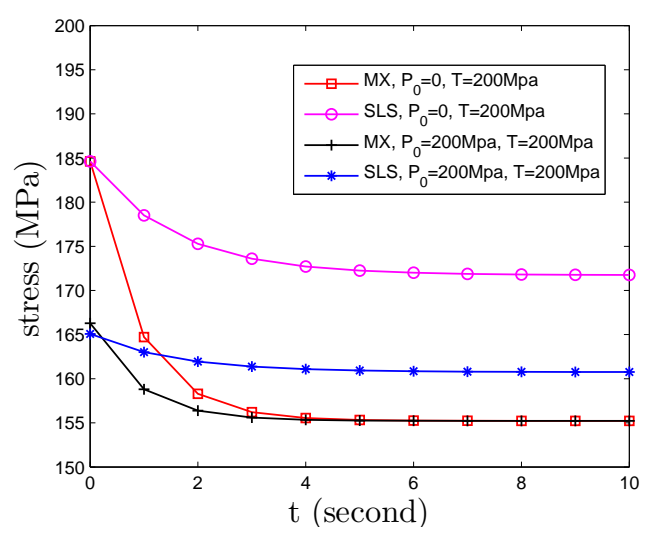

(c) at the matrix $r=1.5 a$ for $2 \mathrm{D}$ case.

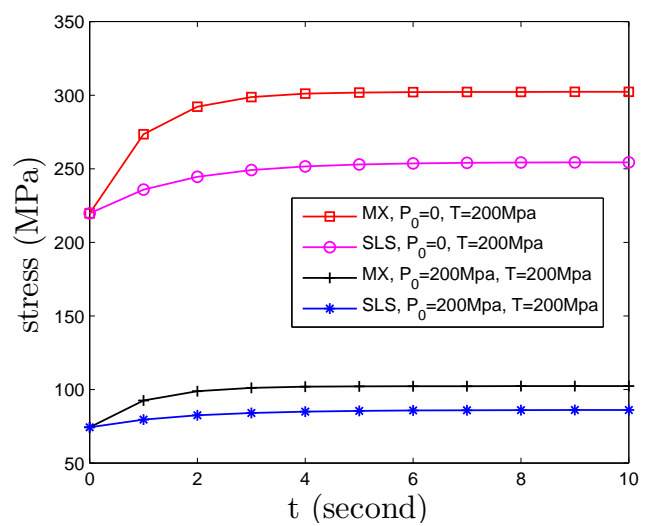

(b) Stresses in the inclusion for 3D case.

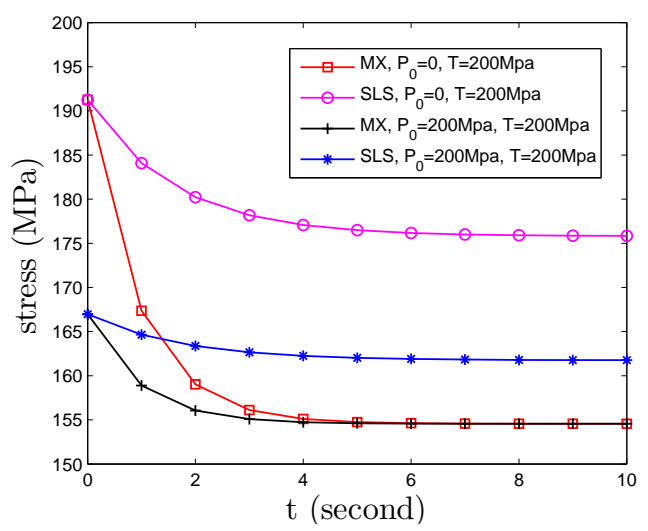

(d) at the matrix $R=1.5 a$ for $3 \mathrm{D}$ case.

Figure 2.3: Hoop stress with and without pre-stressing the inclusion [1].

with the outer core and the mantle. Another interesting example of such a composite is a blood cell which can be modeled as an elastic solid containing a viscoelastic fluid. It suffices to recognize that there are several geological applications wherein one can view the body of interest as a composite comprised of an elastic solid and a viscoelastic fluid constituents. From Eq. 2.10 it is seen that increasing the value of the compressive prestress $P_{0}$ would lead to high compressive stresses in the brittle inclusions, which is desired if the composites are predominantly carrying the external tensile stresses. This can avoid cracking in the brittle inclusions. However, the load carrying capacity in the composite 
can also reduce due to debonding/delamination between the inclusion and matrix. As stated above, debonding occurs due to high stress discontinuities at the interface between the matrix and inclusion, $\triangle \sigma_{\theta \theta}(a, t)=\sigma_{\theta \theta}^{m}(a, t)-\sigma_{\theta \theta}^{i}(a, t)$. Fig. 2.4 shows the hoop stress discontinuities at the interface, at different times, when different values of prestress are prescribed. For the problem under consideration, there is a range of pre-stress values at which the stress discontinuity at the interface is minimum, e.g. $50-200 \mathrm{MPa}$. The result from this case study can help designing composite with improved load carrying capacity.

Furthermore, the stiffness of the constituents also influences the magnitude of stresses in the composites. Figure 2.5 shows the normal stress field in the inclusion and the hoop stress discontinuities at the interface when the viscoelastic matrix has different values of instantaneous modulus. The instantaneous modulus of the viscoelastic matrix only affects the response at early time, as expected. Since the stiffer inclusion takes most of the load from the compliant matrix. The softer the matrix, the smaller the stress discontinuity at the interface is and the less the body experiences relaxation, as expected. Thus, when a brittle and stiffer inclusion is used for composites that are designed to sustain external tensile stresses, prescribing compressive prestress in the inclusion and considering softer or compliant matrix hold promise with regard to enhancing load carrying capacity of composites.

\subsubsection{Response of the composite due to uniaxial tensile load}

The previous studies discuss the response of composites when a uniform tensile stress is prescribed on the entire boundary of the composites. In many applications, composites are often subjected to non-uniform boundary conditions, such as uniaxial loadings. This study considers the effect of prestressing the inclusion on the overall response of composites when the composite system is subjected to a uniaxial tensile load. In such situation, the stresses acting on the inclusion and matrix depend not only on the radial direction, but also vary in the circumferential (hoop) direction. Furthermore, the composite experiences 


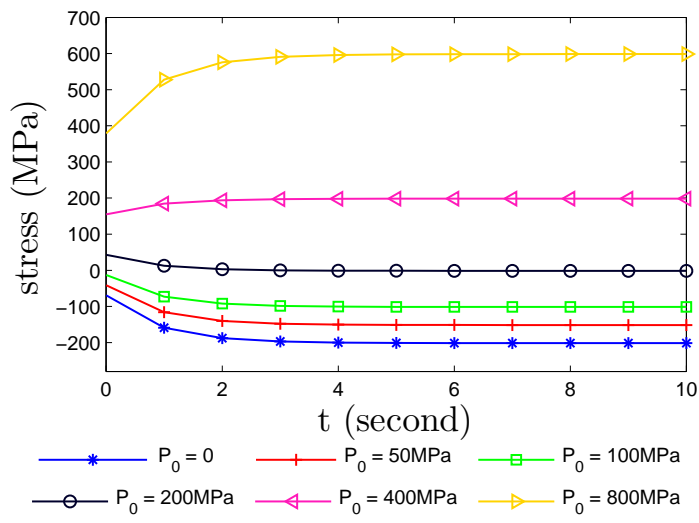

(a) $2 \mathrm{D}$ case

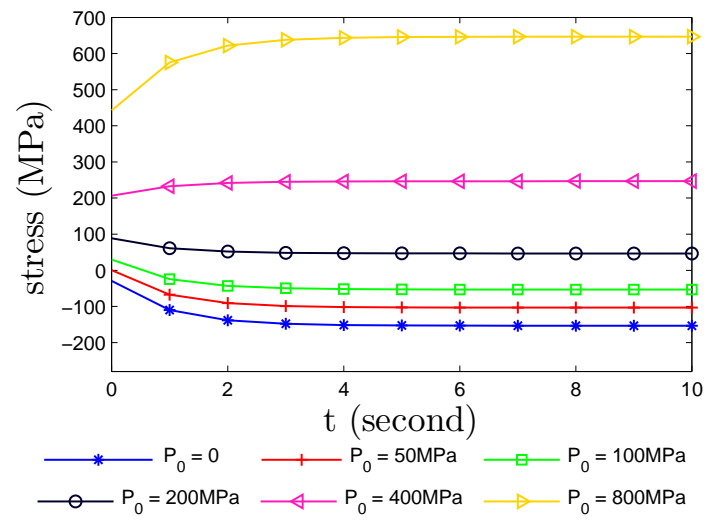

(b) 3D case

Figure 2.4: Hoop stress jump at the interface with external tension $T=200 \mathrm{MPa}$ and various prestresses [1].

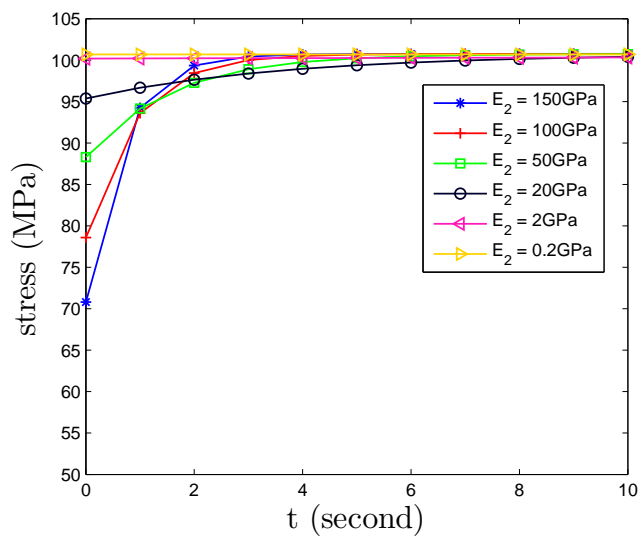

(a) Stress fields in the inclusion

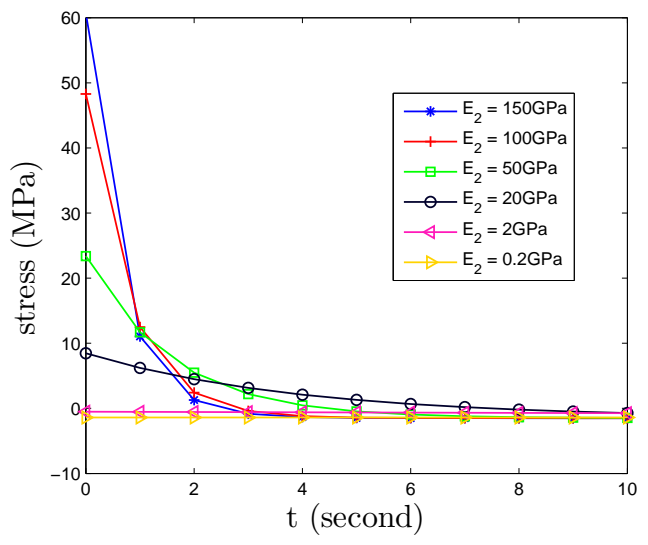

(b) Hoop stress jump at the interface

Figure 2.5: Stresses with external tensile load $T=200 \mathrm{MPa}$ and internal compressive prestress $P_{0}=200 \mathrm{MPa}$ for $2 \mathrm{D}$ case. The Material parameters are $E^{i}=200 \mathrm{GPa}, \nu^{i}=$ $0.25, \nu_{2}=0.33, \mu_{2}=69 \mathrm{GPa} \cdot \mathrm{s}[1]$.

both normal and shear stresses. Figure 2.6 illustrates the maximum principal stresses in the inclusion at two radial locations, $r=0.5 a$ and $r=a$, at time $t=10 \mathrm{~s}$, and at various circumferential direction. The angle $\theta$ is measured starting from the horizontal axis 
pointed to the right direction and going counter clock wise direction up to $\theta=2 \pi \mathrm{rad}$. It is seen that the stresses in the inclusion vary from tension to compression. Figure 2.7 shows the maximum shear stresses at the inclusion, $r=0.5 a$, and at the matrix, $r=1.5 a$. It is seen that the existence of the shear stresses in the constituents could lead to shear failure in the composites. In order to examine the potential of debonding at the interface, the hoop stress discontinuities are also investigated, as shown in Fig. 2.8. Unlike the situation with uniform external tensile load, when the composite is subjected to a uniaxial tensile load the hoop stress discontinuities at the interface between the inclusion and matrix do not necessarily drop with increasing the compressive prestress. At certain angles, e.g. $\theta=0$, the stress discontinuity is small, whereas at other angles, e.g. $\theta=\pi / 2$, the stress discontinuity increases. Although in the case of uniaxial loading, the stress discontinuities would vary at different locations and prestressing the inclusion does not necessarily reduce the entire stress discontinuities, certain choice of prestressing can still give an enhanced performance when taking into account the overall response of the composites. It is seen that it is necessary to consider non-uniform prestress in the case of uniaxial loading as discussed in Zhu et al.[1]. 


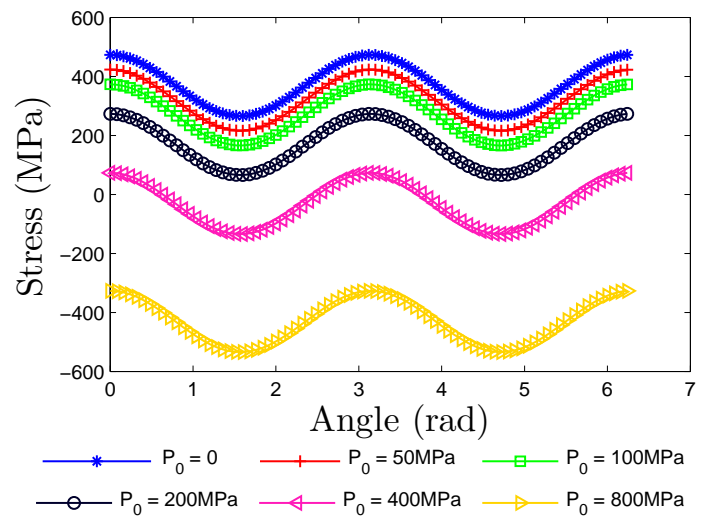

(a)

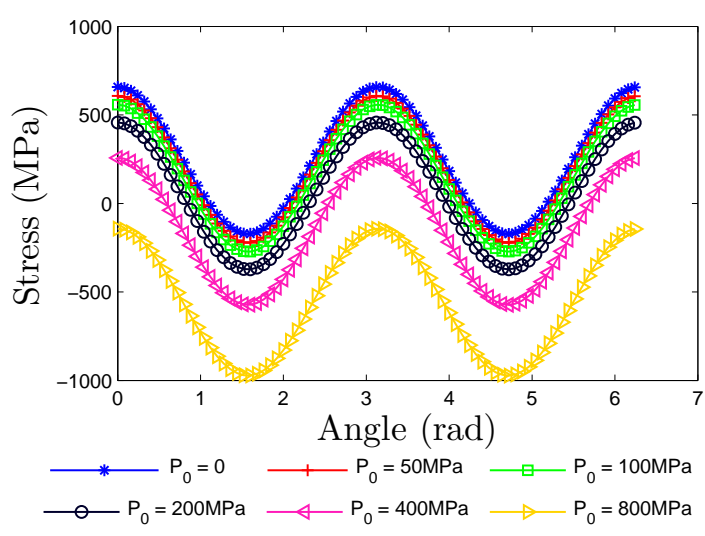

(b)

Figure 2.6: Maximum principal stresses in the inclusion at a) $r=0.5 a$ and b) $r=a[1]$.

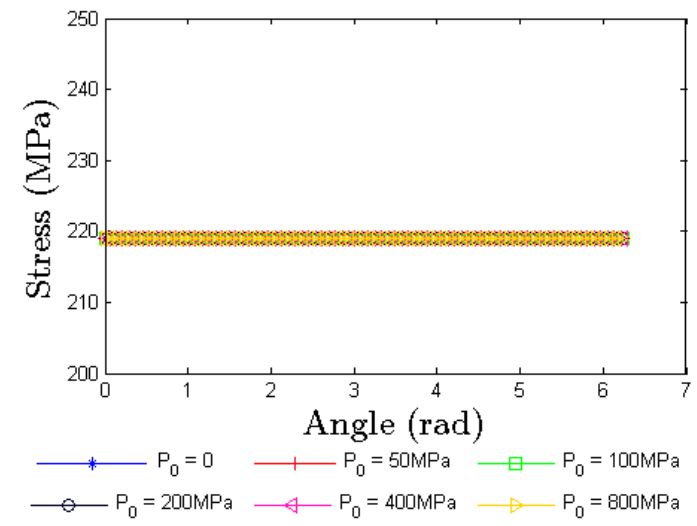

(a)

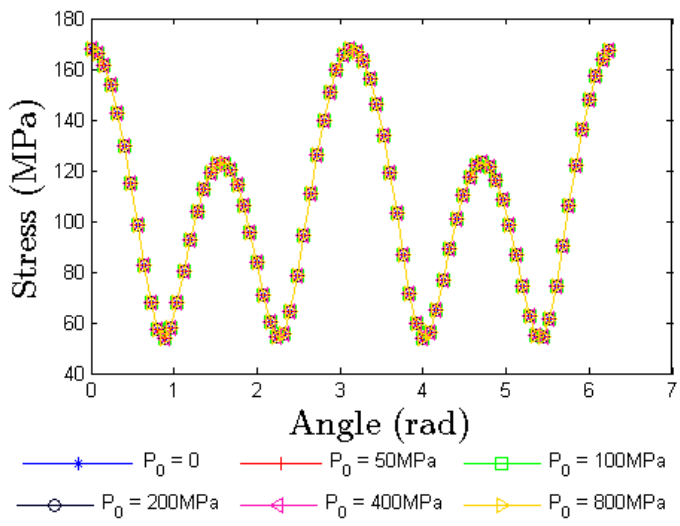

(b)

Figure 2.7: Maximum shear stress a) in the inclusion $r=0.5 a$ and b) in the matrix $r=1.5 a$ [1]. 


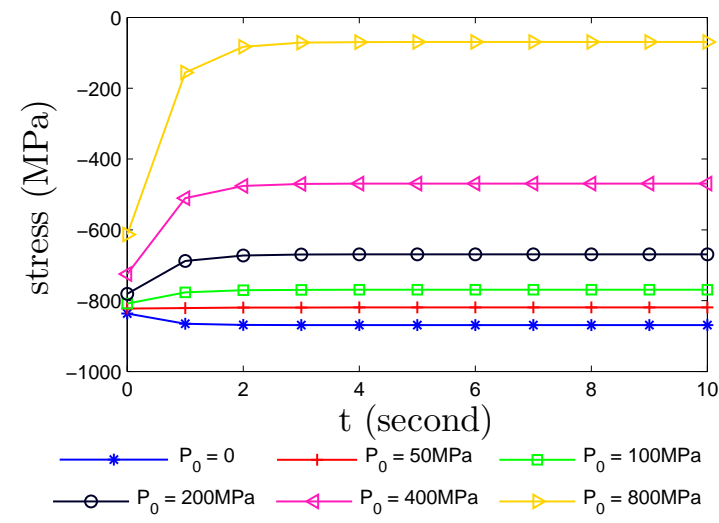

(a)

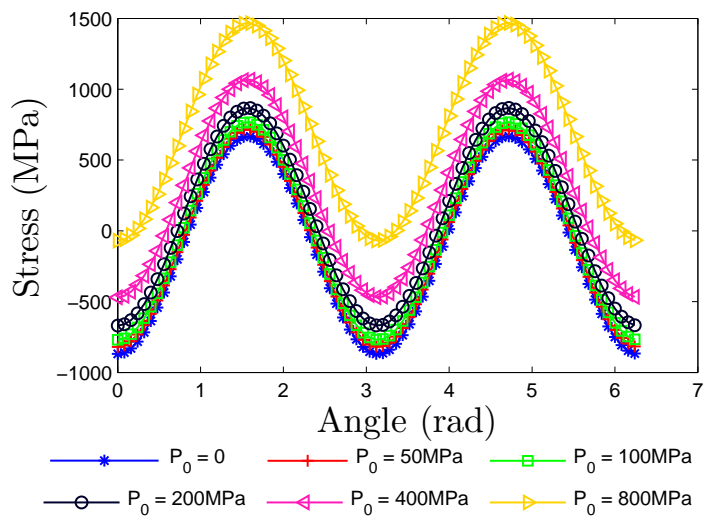

(b)

Figure 2.8: Hoop stress discontinuities at the interface a) at $\theta=0$ and b) at $t=10 \mathrm{~s}$ [1].

\subsubsection{Non-uniform prestress in the case of uniaxial loading}

In the above discussions, we have considered uniform prestresses only. In practice, it might not always possible to obtain uniform prestresses. We have also investigated the composite with a non-uniform prestress condition to examine the overall response of composites under a more general prestress condition. Suppose the prescribed prestress in the inclusion is a non-uniform function of $\theta$. Let's consider the situation when $P(\theta)=$ $P_{0}(1+\cos 2 \theta)$. The maximum principal stress in the inclusion and in the matrix are shown in Fig. 2.9 and Fig. 2.10 at steady state $t=10 \mathrm{~s}$, respectively. Unlike the previous cases for uniform prestress, the stress state transition is not monotonic here and shear stress in the inclusion also changes due to this non-uniform prestress, as depicted in Fig. 2.11. The effect of this non-uniform prestress on the hoop stress discontinuity is displayed in Fig. $2.12 \mathrm{a}$ and $2.12 \mathrm{~b}$. It is confirmed that the hoop stress discontinuity is decreased with the appropriate prestress value. Thus, one can possibly conclude that when the composite is predominantly subjected to uniaxial loading, perhaps non-uniform prestress is required to enhance the overall load carrying capacity of the composite. 


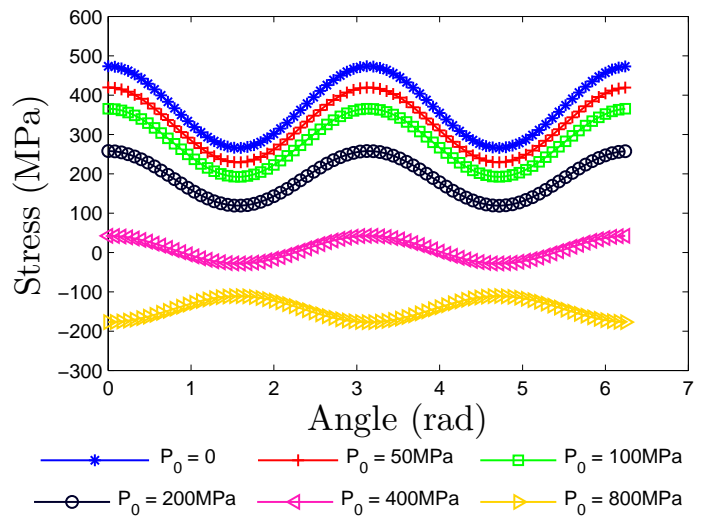

(a)

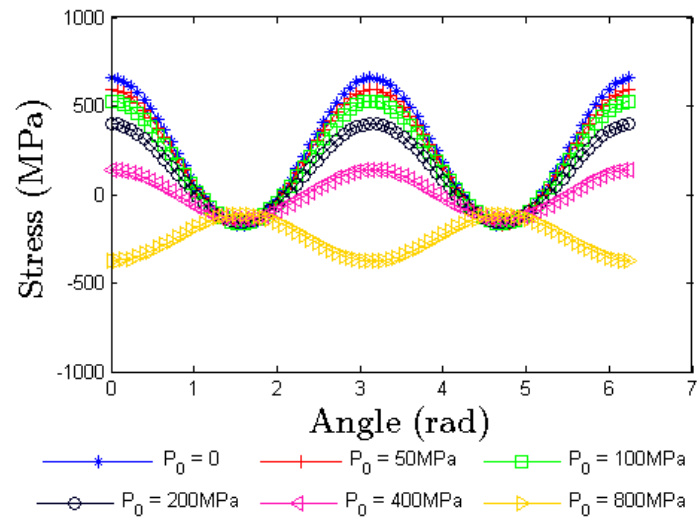

(b)

Figure 2.9: Maximum principal stress in the inclusion at $t=10 \mathrm{~s}$ varying with respect to angle $\theta$. (a) at $r=0.5 a$, (b) at $r=a[1]$.

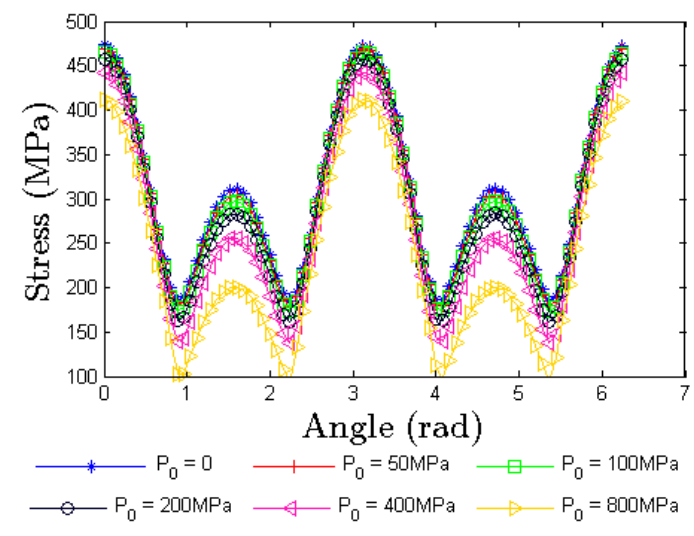

(a)

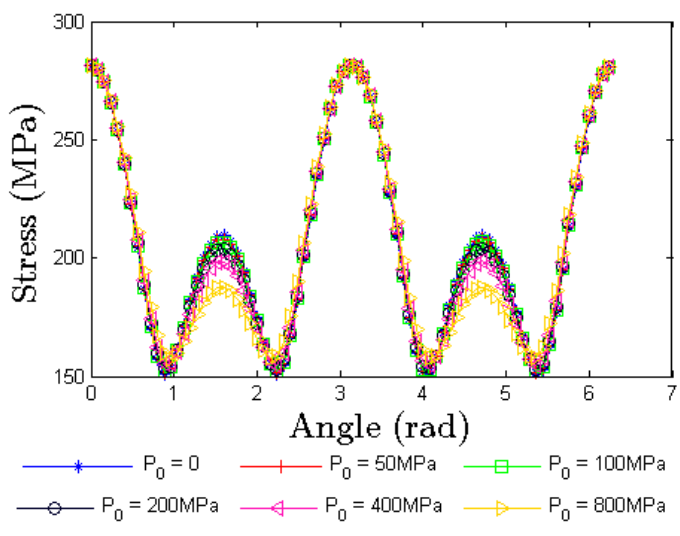

(b)

Figure 2.10: Maximum principal stress in the matrix at $t=10 \mathrm{~s}$ varying with respect to angle $\theta$.(a) at $r=a$, (b) at $r=1.5 a[1]$. 


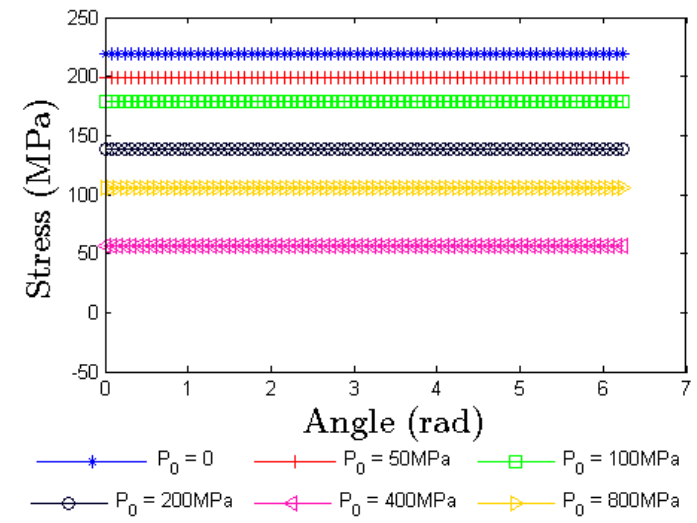

(a)

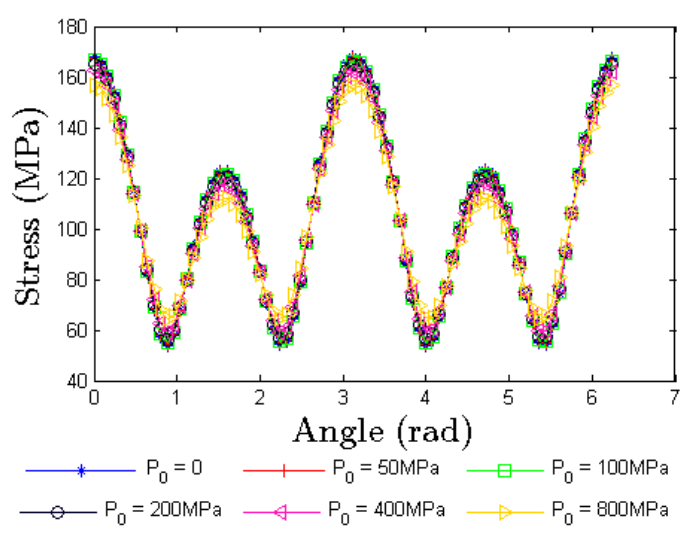

(b)

Figure 2.11: Maximum shear stress. (a) at the inclusion location $r=0.5 a$, (b) at the matrix location $r=1.5 a$ [1].

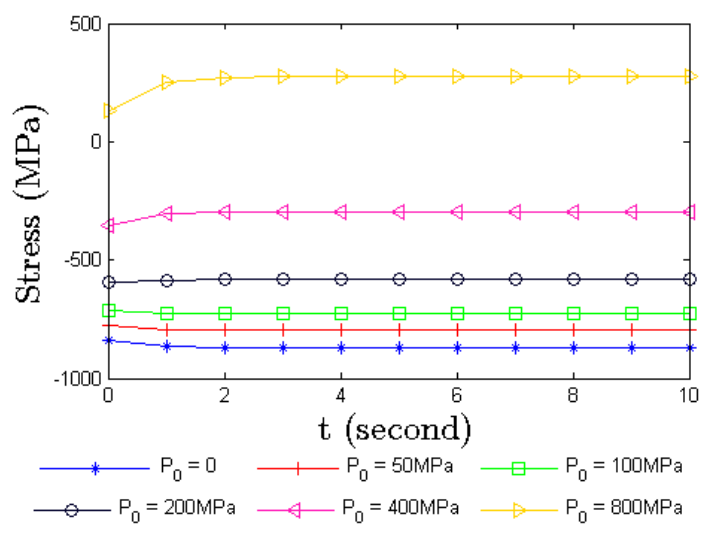

(a)

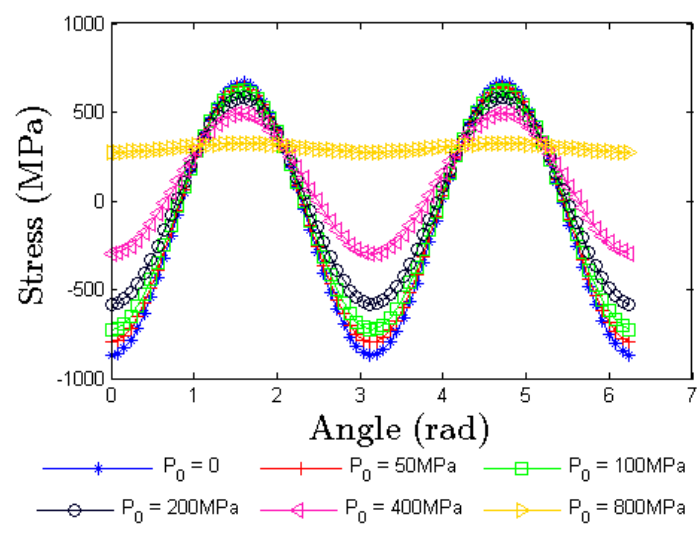

(b)

Figure 2.12: Hoop stress jump at the interface $r=a$. (a) at $\theta=0$, (b) at $t=10 \mathrm{~s}$ [1].

\subsubsection{Prestressing ductile inclusion in a brittle matrix}

There are other types of composites which comprise of a ductile inclusion embedded in a brittle matrix, such as adding rubber particles to the glassy polymers or using ductile in- 
clusions in the brittle ceramic matrix in order to improve ductility of the composites. This case study deals with a composite with a ductile inclusion placed in an infinite medium of a brittle matrix, and the composite is subjected to a uniform compressive stress, $T=-200$ $\mathrm{MPa}$, on its boundary. Like in the previous cases, both inclusion and matrix are assumed isotropic, homogeneous, and undergo small deformation, and the interface between the inclusion and matrix is perfectly bonded. The inclusion is modeled as a Maxwell viscoelastic fluid while the matrix is assumed to be linearized elastic solid. The properties for the Maxwell viscoelastic fluid and elastic solid from Table 3.1 are used. Figure 2.13a depicts the time-dependent normal stresses in the inclusion at various values of prestress. Both tensile and compressive prestresses are considered. It is seen that significant compressive stress relaxation occurs in the viscoelastic inclusion, which should be expected. The hoop stresses in the brittle matrix at the location $r=1.5 a$ is shown in Fig. 2.13b. It is shown that prestressing the inclusion in this case does not have significant effect on the stress response of the viscoelastic inclusion as well as the elastic matrix. The hoop stress discontinuities at the interface, shown in Fig. 2.14, indicates that the stress discontinuities reach a minimum value when the prestress is in the range of $400-800 \mathrm{MPa}$ in this example. 


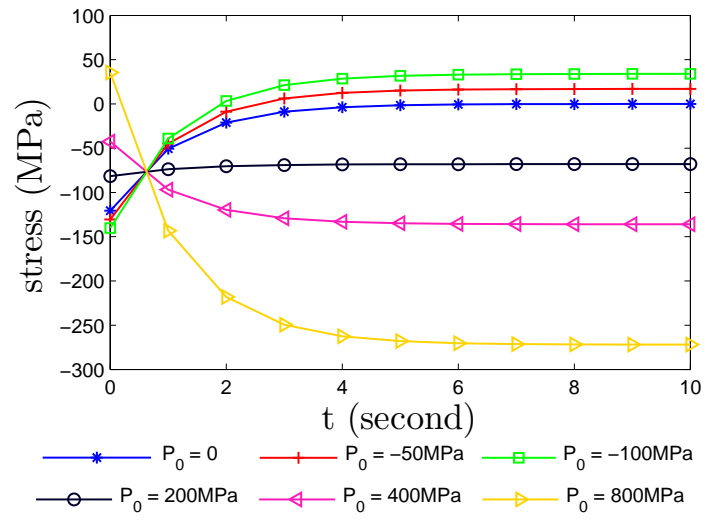

(a) Stress fields in the inclusion

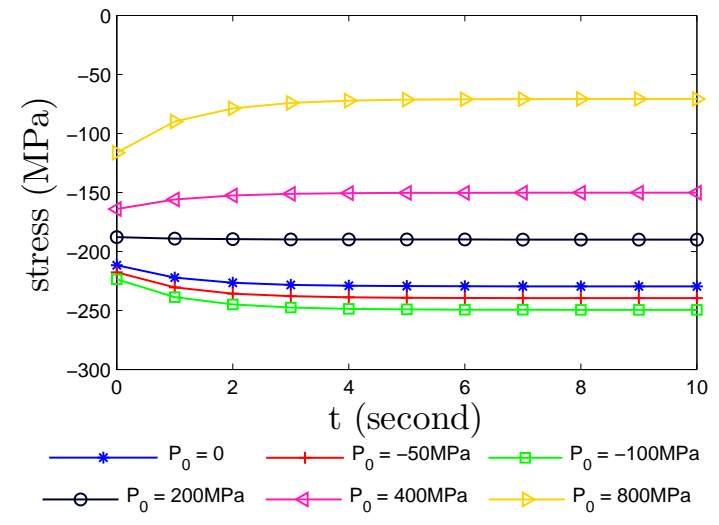

(b) Hoop stress at $R=1.5 a$

Figure 2.13: Stress fields in the inclusion and matrix at various values of prestresses [1].

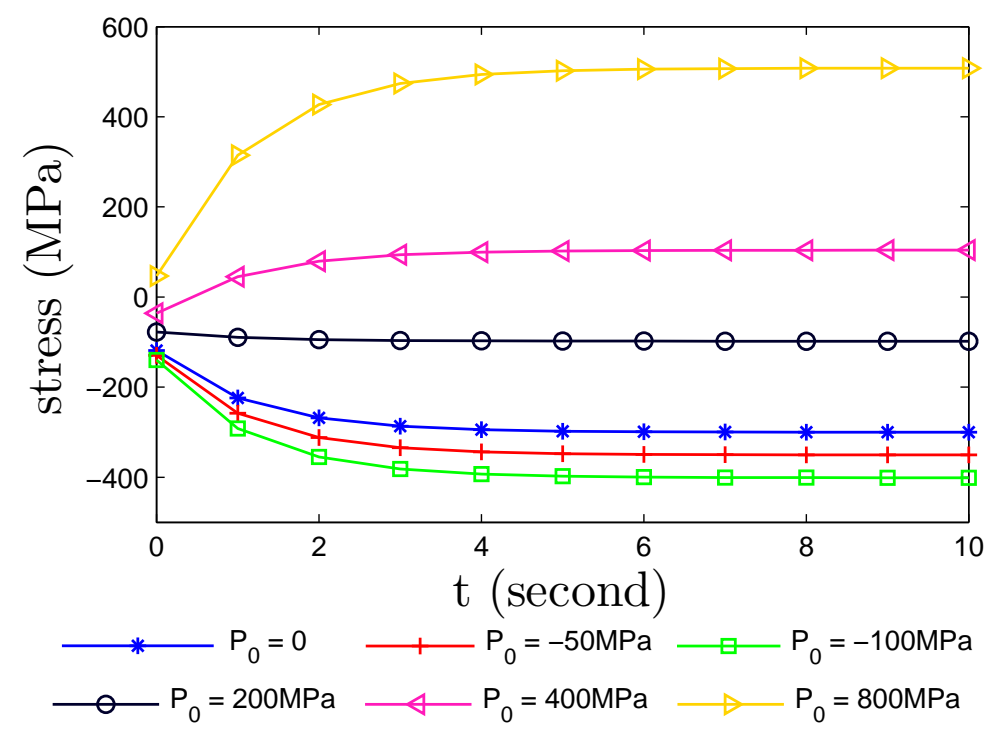

Figure 2.14: Hoop stress discontinuities at the interface with different values of prestress [1]. 


\section{STUDIES OF INTERFACE/INTERSTITIAL MATTER: FE ANALYSIS}

In reality, composites have finite dimensions and comprise of several particle arrangements dispersed in matrix medium and the microstructures might also contain other inhomogeneities, such as voids, cracks, and other constituents due to chemical reactions during the processing. When one wants to model and analyze response of composites, several simplification and approximation are often made in order to reduce complexity as it might not be possible or even necessary to include all inhomogeneities in characterizing the overall response of the composites. In most cases, it might not be possible to obtain exact closed form solutions in predicting the response of the composites when certain complexity is considered. For this purpose, numerical solutions are often sought. Finite element (FE) method is commonly used to obtain approximate solutions to predict the overall response of composites when a more complex microstructure is considered. This section discusses FE analysis of composites due to a prestress effect by incorporating more realistic microstructures, but still within certain simplification and approximation.

\subsection{Analytical/Numerical solutions}

In the previous section, the theoretical analysis for one inclusion embedded in an infinite medium are provided. The results using numerical method for finite medium is investigated and the numerical results are compared to those of theoretical solutions. Meantime, the comparison with analytical solutions justify the numerical analysis and further makes the numerical analysis of composites with more realistic microstructures and material models meaningful. The stress fields of the finite medium composite, comprising of one inclusion with radius $r=0.001 \mathrm{~mm}$, are depicted with markers in Fig. 3.1 and Fig. 3.2, representing 2D and 3D, respectively. The solid line shows the corresponding theoretical results for an infinite medium. As expected, the numerical results for finite 
matrix converge to the analytical results when the dimension ratio of matrix to inclusion approaches high value(here, 10).
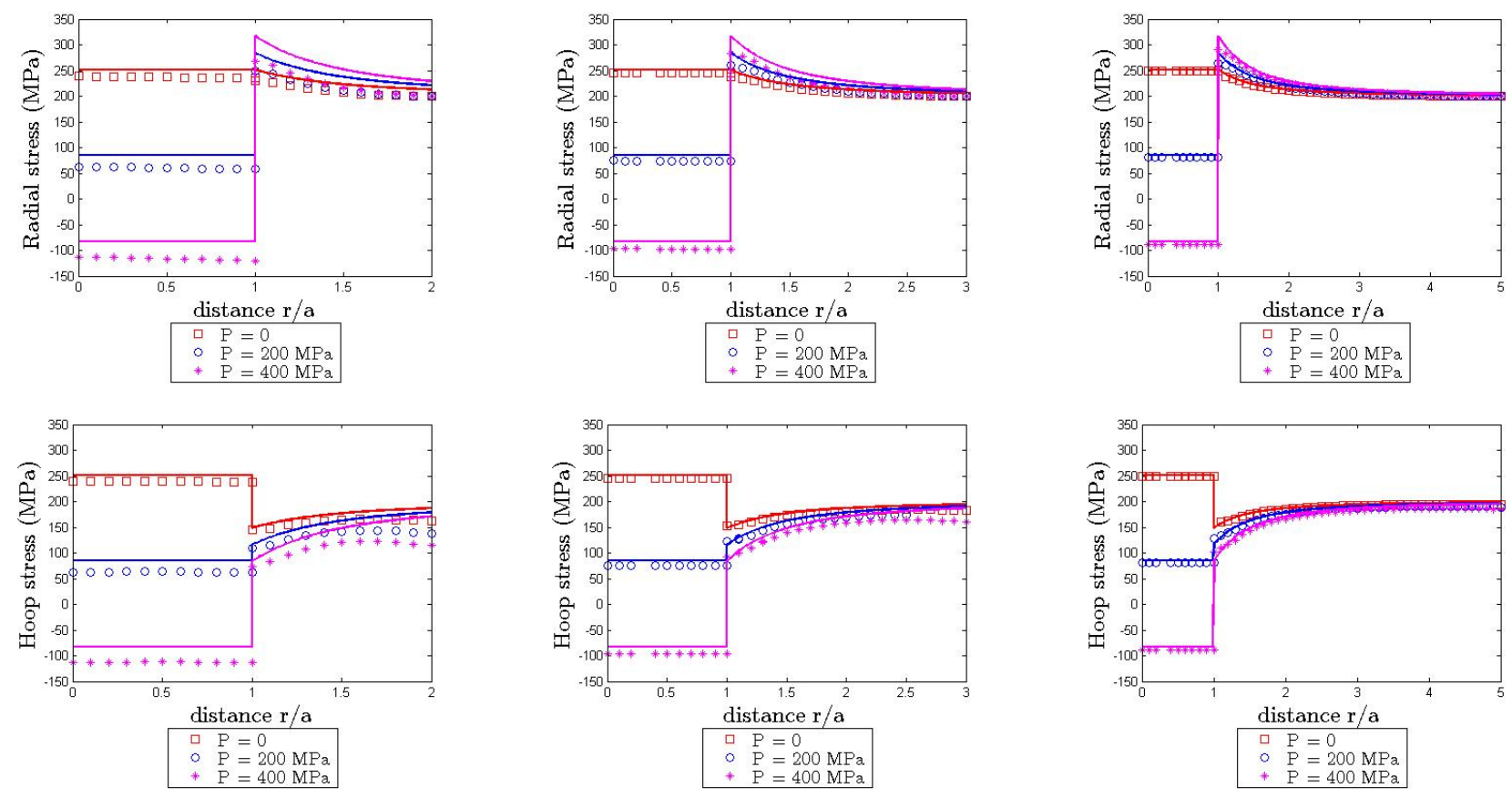

Figure 3.1: Stress field with various matrix dimension in 2D. Dimension of the matrix from left to right: $4 \mathrm{~mm}, 6 \mathrm{~mm}, 10 \mathrm{~mm}$. 

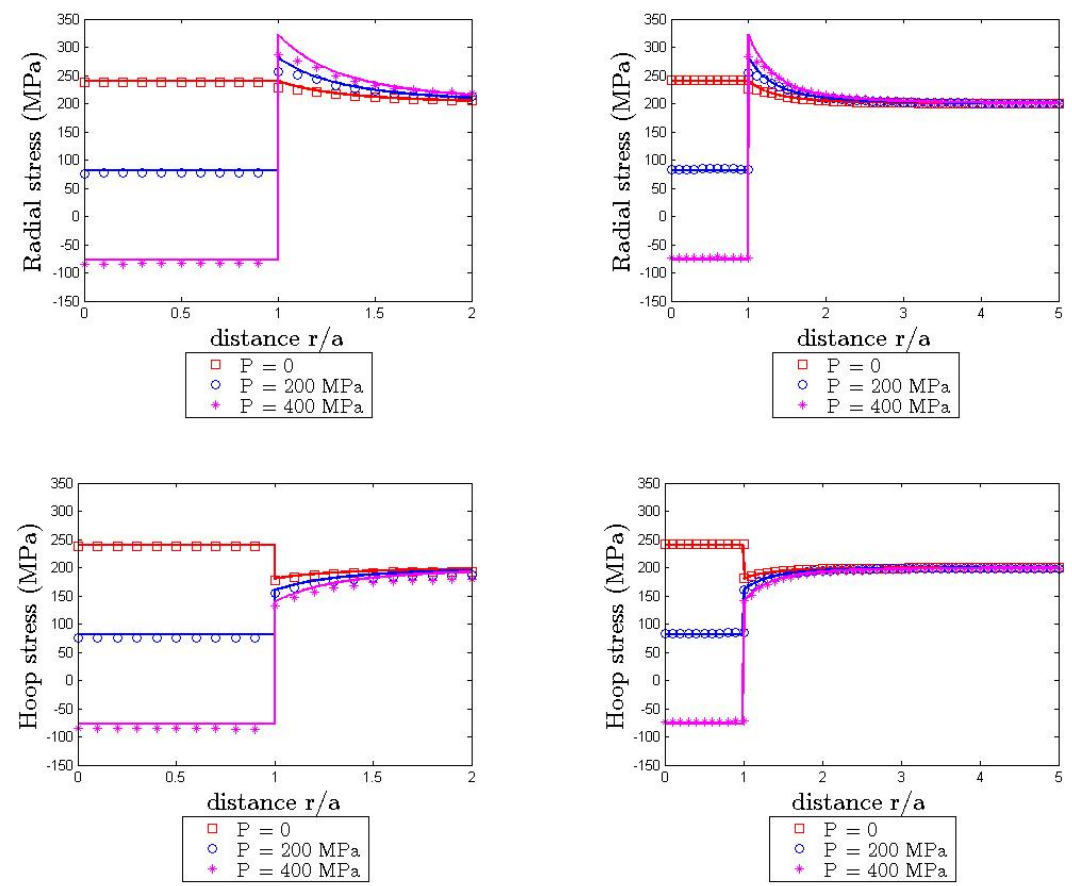

Figure 3.2: Stress field with various matrix dimension in 3D. Dimension of the matrix from left to right: $4 \mathrm{~mm}, 10 \mathrm{~mm}$.

\subsection{Finite element analysis}

Consider a 3D cubic domain of a composite with a side length of $0.01 \mathrm{~mm}$. The composite contains uniform solid spherical inclusions, each of radius $0.001 \mathrm{~mm}$, dispersed randomly in the matrix domain. Figure 3.3 illustrates the composite microstructures, having particle and matrix constituents, with $10 \%$ and $30 \%$ particle volume contents. These non-overlapping identical spheres are randomly and sequentially generated in the matrix using a new, modified random sequential adsorption algorithm suitable for particle volume fractions up to $50 \%[46]$.

We use the algorithm to generate the coordinates of the particle centers for composites having $10 \%$ and $30 \%$ volume fractions. During the generation, the particles are allowed 
to intersect with the matrix surface. The interfaces between particles and matrix are assumed perfect. Composites could also contain a small region (interstitial matter) between the particle and matrix, which is considered as a third constituent. Interstitial matter could be formed from the reaction process between the inclusions and matrix during the manufacturing of the composites, or surface treatment or coating could also be performed on the inclusions in order to enhance the bond strength between the inclusions and matrix. This study also considers composite microstructures with interstitial regions between the particles and matrix, as shown in Fig. 3.3. The thickness of the interphases is assumed uniform, which is $0.0002 \mathrm{~mm}$.

FE analysis are conducted on composites, having alumina (brittle) particles of a linear elastic material model dispersed in FM73 polymeric matrix of viscoelastic behavior, subjected to a uniform tensile load on the boundaries of the composites. The properties of the alumina, interstitial matter, and FM73 polymer [47] are listed in Tables 3.1 and 3.2. The interstitial matters are assumed as linear elastic. It is noted that experimentally characterizing the properties and response of the interstitial matter is rarely done, and the interstitial matter would strongly depend on the processing method, and the inclusions and matrix used in the composites. Since the properties of interstitial matters are not readily available in the literature, parametric studies are conducted on examining the effects of interstitial matters of various properties and prestress on the overall response of composites. The composite is meshed with quadratic tetrahedral element C3D10. Mesh convergence study are depicited in Fig. 3.4. 


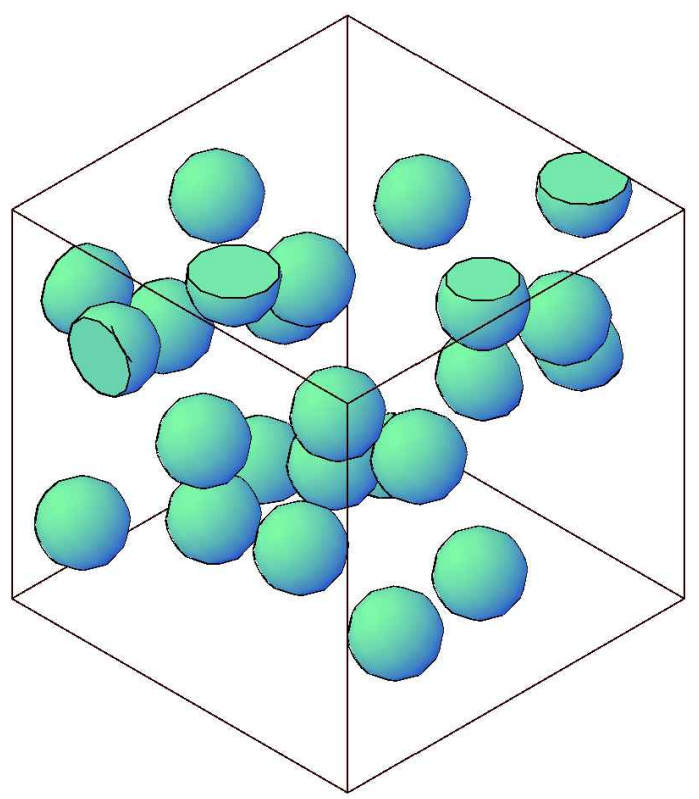

(a) 10 percent volume fraction

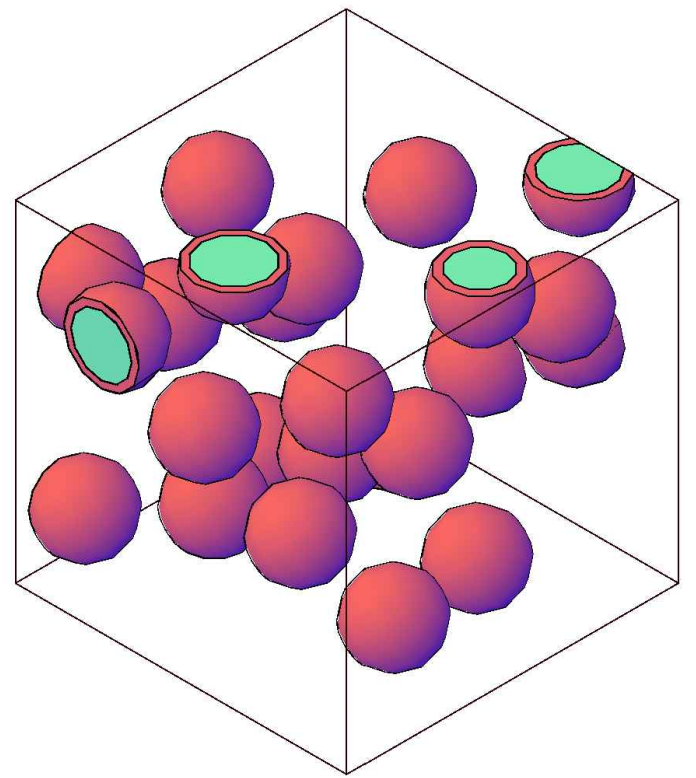

(c) 10 percent volume fraction

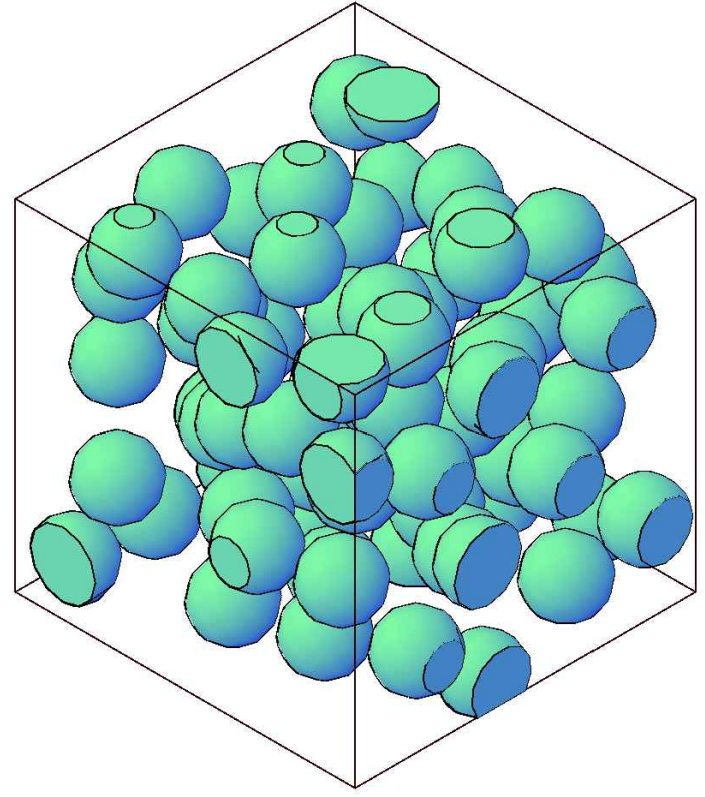

(b) 30 percent volume fraction

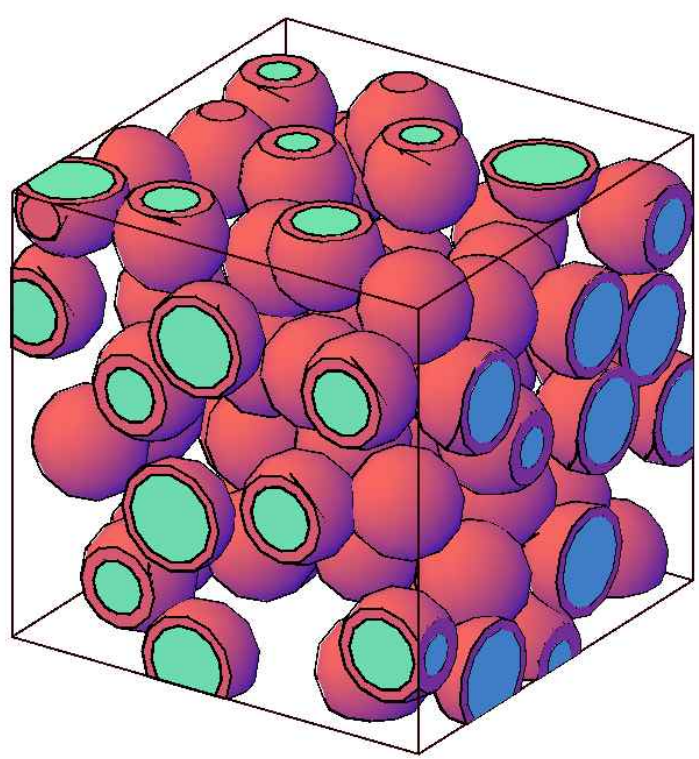

(d) 30 percent volume fraction

Figure 3.3: Composite model with various particle volume fractions. 


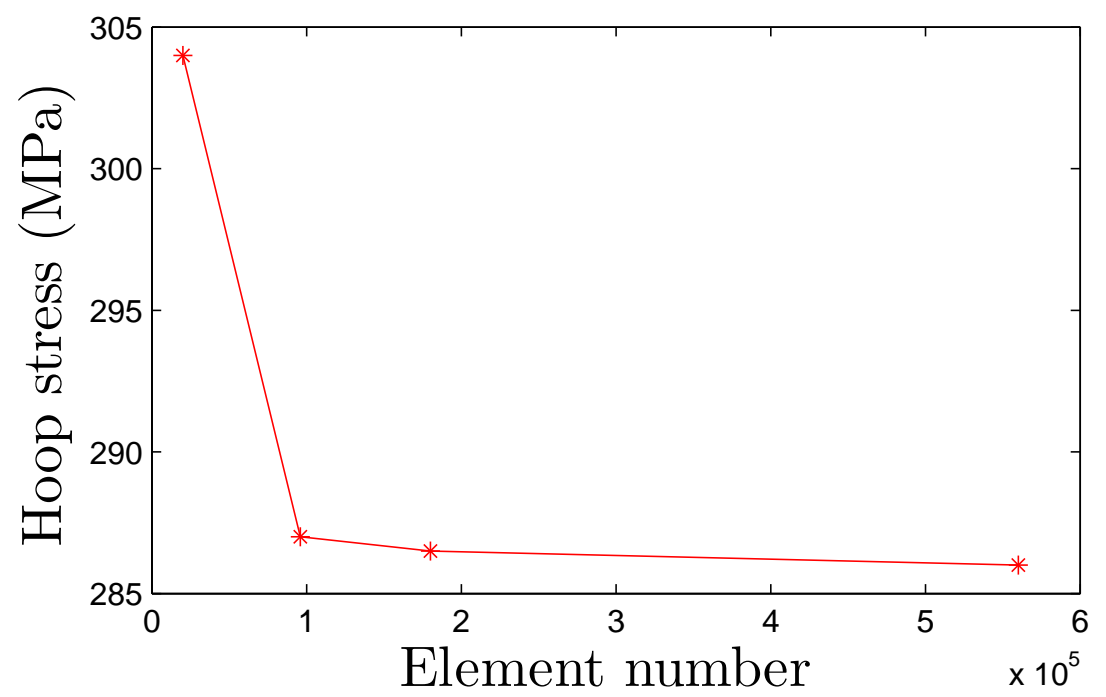

Figure 3.4: Mesh convergence study.

Table 3.1: Elastic properties and Prony series coefficients for the matrix [2].

\begin{tabular}{ccc}
\hline \hline$n$ & $\lambda_{n}\left(s^{-1}\right)$ & $D_{n} \times 10^{-6}(\mathrm{MPa})^{-1}$ \\
\hline 1 & 1 & 21.0 \\
2 & $10^{-1}$ & 21.6 \\
3 & $10^{-2}$ & 11.8 \\
4 & $10^{-3}$ & 15.9 \\
5 & $10^{-4}$ & 21.6 \\
6 & $10^{-5}$ & 20.0 \\
$E=2710 \mathrm{MPa}$ & $\nu=0.35$ & \\
\hline
\end{tabular}


Table 3.2: Linear thermo-elastic properties of constituents.

\begin{tabular}{c|ccc}
\hline \hline Constituents & $E(\mathrm{GPa})$ & $\nu$ & $\alpha\left(/{ }^{0} \mathrm{C}\right)$ \\
\hline Inclusions & 200 & 0.25 & $4.6 \times 10^{-6}$ \\
Matrix & 2.71 & 0.35 & $6.5 \times 10^{-5}$ \\
Stiff interstitial matter & 400 & 0.25 & - \\
Soft interstitial matter & 1.0 & 0.25 & $2.0 \times 10^{-4}$ \\
\hline
\end{tabular}

\subsection{Constitutive model}

The Boltzmann integral model for linear viscoelasticity of non-aging matrix is considered. The uniaxial viscoelastic constitutive relation with fixed environmental conditions is expressed as[41]:

$$
\varepsilon(t)=D_{0} \sigma(t)+\int_{0^{-}}^{t} \Delta D(t-\tau) \frac{\mathrm{d} \sigma(\tau)}{\mathrm{d} \tau} \mathrm{d} \tau
$$

where $D_{0}$ is the uniaxial instantaneous elastic compliance and $\triangle D$ is the transient compliance given by Prony series as:

$$
\triangle D(t)=\sum_{n=1}^{N} D_{n}\left(1-\exp \left[-\lambda_{n} t\right]\right)
$$

This one-dimensional uniaxial relation is generalized to three-dimensional multi-axial constitutive model by separating the deviatoric and volumetric strain-stress relations for 
isotropic material behaviors.

$$
\begin{aligned}
e_{i j}^{t} & =\frac{1}{2} J_{0} S_{i j}^{t}+\frac{1}{2} \int_{0^{-}}^{t} \triangle J(t-\tau) \frac{\mathrm{d} S_{i j}(\tau)}{\mathrm{d} \tau} \mathrm{d} \tau \\
\varepsilon_{k k}^{t} & =\frac{1}{3} B_{0} \sigma_{k k}^{t}+\frac{1}{3} \int_{0^{-}}^{t} \triangle B(t-\tau) \frac{\mathrm{d} \sigma_{k k}(\tau)}{\mathrm{d} \tau} \mathrm{d} \tau
\end{aligned}
$$

where $e_{i j}, \varepsilon_{k k}, S_{i j}$ and $\sigma_{k k}$ represent the deviatoric strains, volumetric strain, deviatoric stress and volumetric stress respectively. The parameters $J_{0}, B_{0}, \triangle J$ and $\triangle B$ are the instantaneous elastic shear, bulk, transient shear, bulk compliances respectively. We assume contraction ratio, $\pi$, is time-independent for the matrix, thus, we can express the shear and bulk compliance as:

$$
\begin{aligned}
& J_{0}=2(1+\pi) D_{0} \quad B_{0}=3(1-2 \pi) D_{0} \\
& \triangle J=2(1+\pi) \triangle D \quad \triangle B=3(1-2 \pi) \triangle D
\end{aligned}
$$

A recursive algorithm, which is compatible with general displacement based FE structural analyses, is employed to solve the integral in Eq.(3.3). At the FE structural iteration, the recursive-iterative algorithm is called at each material point for $3 \mathrm{D}$ continuum elements to achieve structural and material convergence simutaneously. Detailed derivation of the recursive-iterative algorithm can be found in [2] and [48]. For the iterative procedure, the Newton-Raphson's(NR) method is used. A solution of a variable $\mathbf{x}$ that satisfies $\mathbf{F}(\mathbf{x})=\mathbf{0}$ is solved by:

$$
\mathbf{x}^{(k+1)}=\mathbf{x}^{(k)}-\left[\frac{\partial \mathbf{F}\left(\mathbf{x}^{(k)}\right)}{\partial \mathbf{x}^{(k)}}\right]^{-1} \mathbf{F}\left(\mathbf{x}^{(k)}\right)
$$

Converged solutions: $\|\mathbf{R}\|=\|\mathbf{F}\| \rightarrow 0$.

This procedure requires that $\mathbf{F}(\mathbf{x})$ and $\frac{\partial \mathbf{F}(\mathbf{x})}{\partial \mathbf{x}}$ are continuous near solution $\mathbf{x}$ and $\frac{\partial \mathbf{F}(\mathbf{x})}{\partial \mathbf{x}}$ 
should not approach zero or infinite. The residual tensor in a total strain formulation is:

$$
\mathbf{R}_{i j}^{t,(k)}=\mathbf{e}_{i j}^{t,(k)}+\frac{1}{3} \varepsilon_{k k}^{t,(k)}-\hat{\varepsilon}_{i j}^{t}
$$

where $\boldsymbol{e}_{i j}^{t,(k)}$ and $\varepsilon_{k k}^{t,(k)}$ are the deviatoric and volumetric strain tensors at the iteration $k$ in the material level, respectively. $\hat{\varepsilon}_{i j}^{t}$ is the current strain from the structural level.

The consistent tangent stiffness matrix $C_{i j k l}^{t}$ at the converged state is:

$$
\boldsymbol{C}_{i j k l}^{t}=\frac{\partial \triangle \boldsymbol{\sigma}_{i j}^{t}}{\partial \triangle \hat{\boldsymbol{\varepsilon}}_{k l}^{t}}=\left[\frac{\partial \boldsymbol{R}_{i j}^{t}}{\partial \boldsymbol{\sigma}_{k l}^{t}}\right]^{-1} ; \quad\left\|\boldsymbol{R}_{i j}^{t}\right\| \rightarrow \mathbf{0}
$$

3.4 The effect of particle volume contents and properties of the interstitial matters

This study investigates the stress distributions in the composites, with and without prestressing the inclusions. Consider composites with $10 \%$ and $30 \%$ particle volume contents, subjected to a tri-axial tensile stress of $60 \mathrm{MPa}$ on the boundary. Figure 3.5 illustrates the maximum principal stress distributions in the particles, at time $=10 \mathrm{~s}$, when the particles are under a uniform compressive pre-stress of $200 \mathrm{MPa}$. The responses are also compared to the ones without pre-stress. As expected, prestressing the brittle inclusions would induce compressive stresses in the inclusions, while its effect on the stress distributions in the matrix (Fig. 3.5) is negligible. It is also seen that in the composite with higher particle contents, there are some localized stresses both in the particles and matrix, which could be due to relatively small distances between particles in the matrix.

Next, the effect of properties of the interstitial matters, whether stiff or compliant, on the overall response of composite is investigated. The properties of the interphases are given in Table 3.2. The interfaces between the particles, interphases, and matrix are assumed perfectly bonded. Figure 3.6 illustrates the stress distributions in the particles, 
interphases, and matrix. It is seen that the role of the interphases could be significant in altering the overall performance of the composites. When the interphases are stiffer than the particles the matrix experiences higher stresses as compared to the composites with soft interphases. The interphases also influence the amount of the compressive stresses in the brittle particles. The corresponding maximum strain contours for the composites with stiff and soft interphases, at early and later times, are presented in Fig. 3.7. As expected these strains increase with times due to the viscoelastic matrix. It is noted that the stress contours remain nearly the same as a result of imposing equilibrium conditions. Continuously increasing strains with time could eventually also lead to delamination between inclusions and matrix.

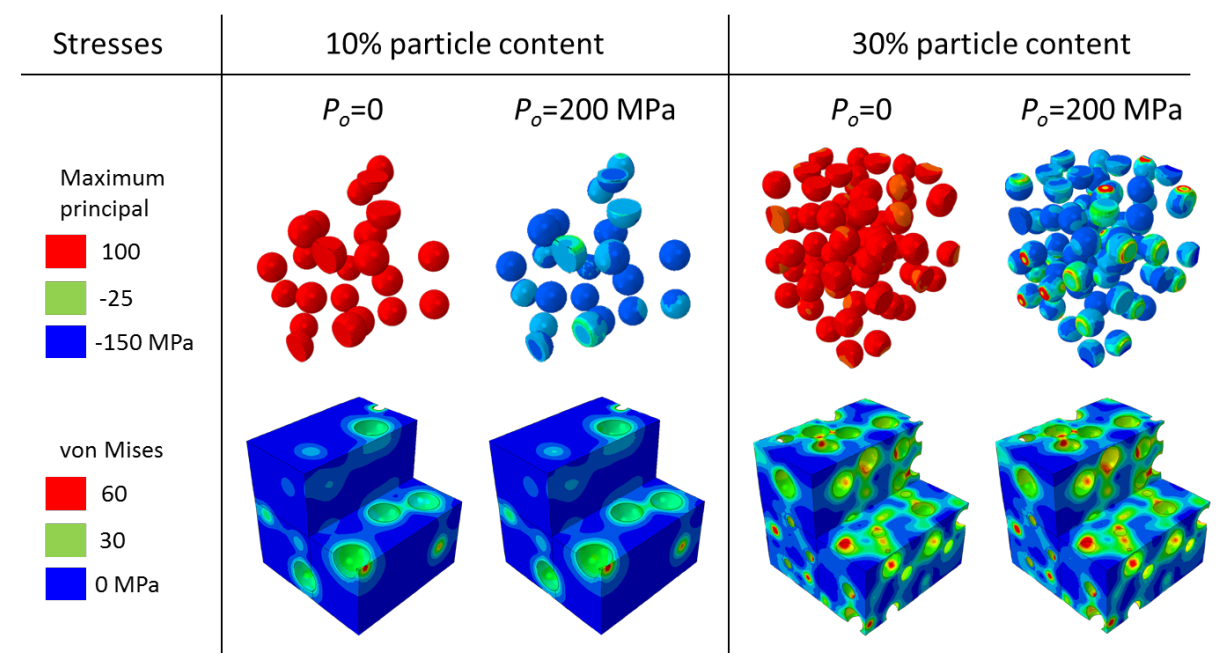

Figure 3.5: The effect of particle volume contents on the overall response of composites $(t=10 s)$. 


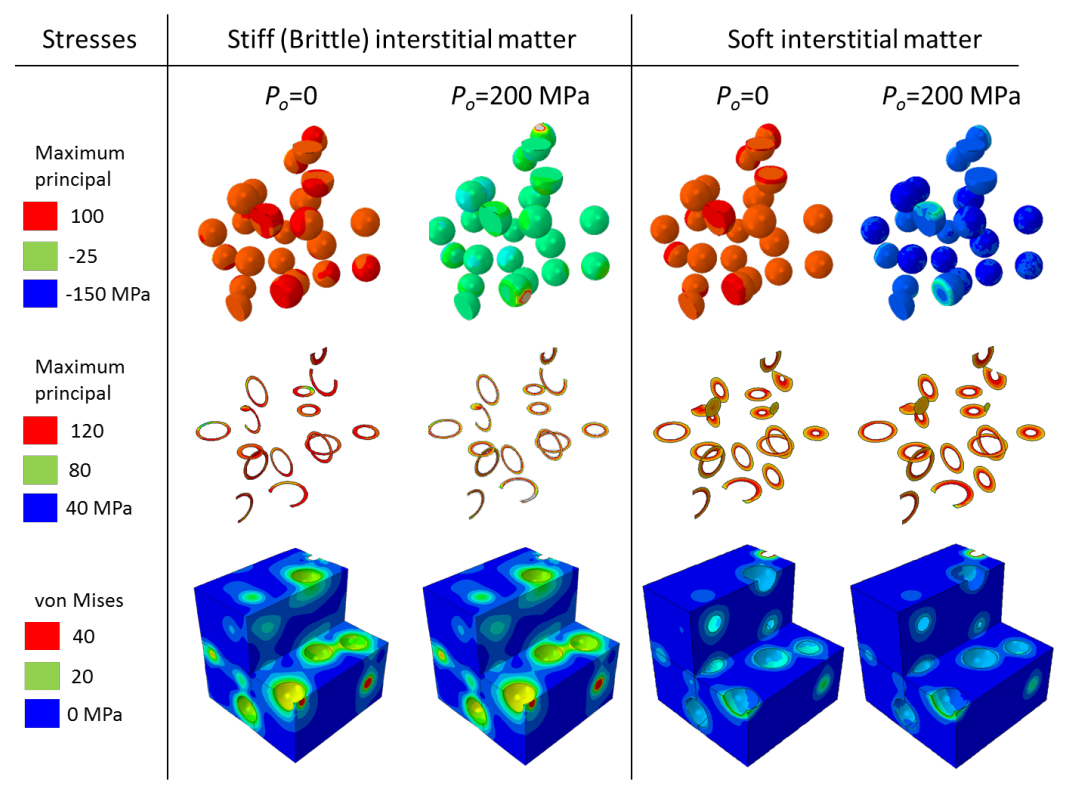

Figure 3.6: The effect of stiff and soft interphases on the overall response of composites with $10 \%$ particle volume content $(t=10 s)$. Top: inclusions; middle: interstitial matters; bottom: matrix.

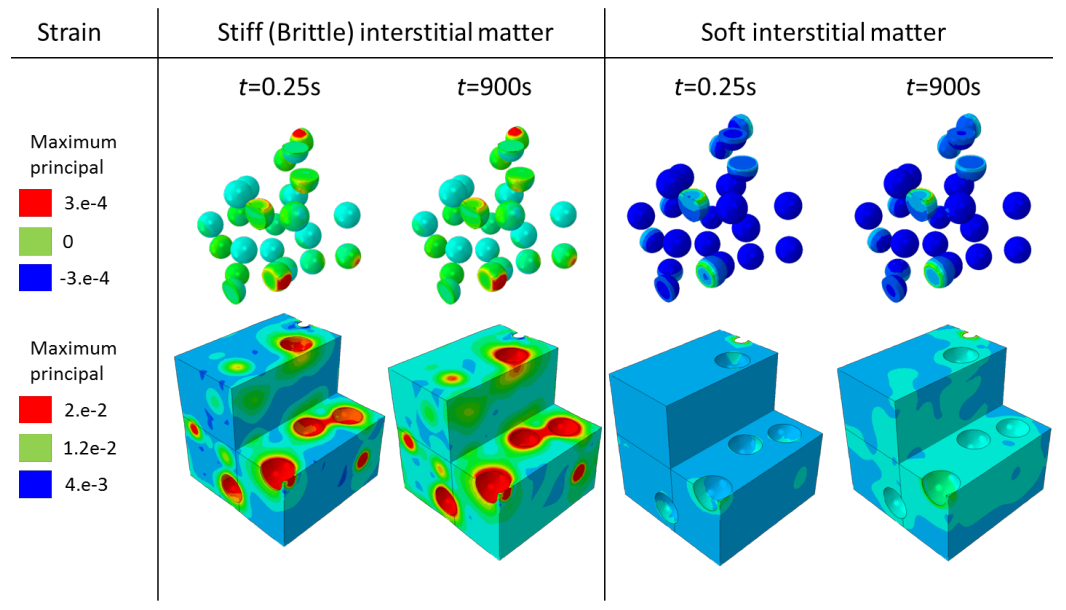

Figure 3.7: Maximum principal strain contours in composites with $10 \%$ particle volume content at early and later times. Top: inclusions; bottom: matrix. 


\subsection{Prestressing particles through thermo-mechanical process}

The above studies considered the effect of prestress and constituent behaviors on the overall performance of composites. From the understanding of the response of composites, if we know characteristic of the constituents, different pre-stressing method can be employed like thermal-process, which is by heating and cooling to induce thermal(pre-) prestress, and autofrettage, which means high presssure applied to induce plastic deformation and upon removal of this pressure residual(pre-) stresses are developed.

This study will present a possible method to induce prestress on the inclusions by temperature changes. Consider a composite comprising of brittle inclusions coated by soft polymers, called soft interphases, dispersed in polymeric matrix. The linear thermo-elastic properties of the constituents are given in Table 3.2. When a processing method involves curing at an elevated temperature, e.g., $225{ }^{0} \mathrm{C}$, upon cooling down to room temperature, the mismatches in the thermal expansion coefficients $(\alpha)$ of the inclusions, interphases, and matrix induce compressive prestresses to the inclusions. Figure 3.8 shows the contours of the maximum principal stresses in the constituents at room temperature, after the cooling process. It is seen that all inclusions are under compressive stresses, the soft interphases experience tension, while the matrix is under nearly zero stresses. It is also observed that the shear stresses in the constituents are negligible. This study shows a possibility in prestressing the inclusions through temperature changes. Fig. 3.9 depicts the maximum principal stress with and without thermal process resisting the same tensile loading. Due to the existence of the compressive residual(pre-) stress in the inclusion, under the same loading, the stress fields in the inclusion reduced and the effect to the matrix constituent is relatively small. 


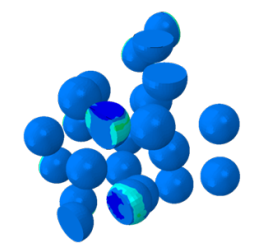

a) particles

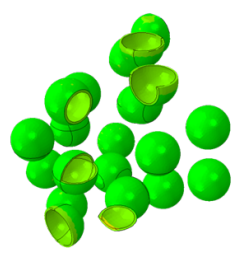

b) Interstitial matter

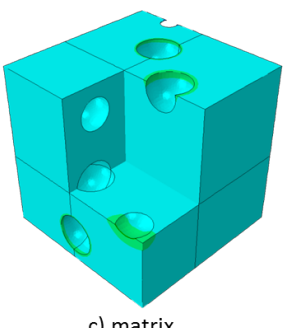

c) matrix

\begin{tabular}{l|l|l}
100 & $30 \square$ & $-40 \mathrm{MPa}$
\end{tabular}

Figure 3.8: Maximum principal stresses in the constituents due to a temperature change.

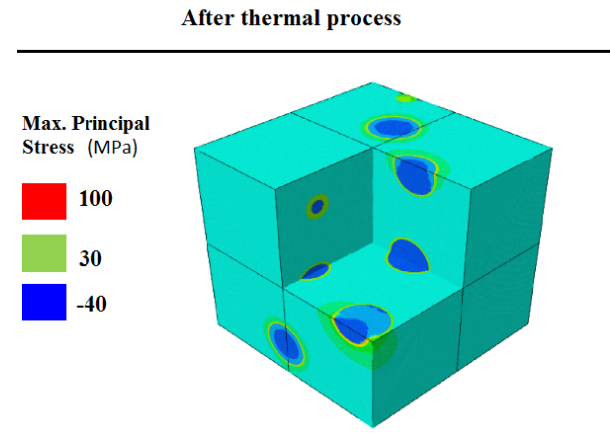

(a)

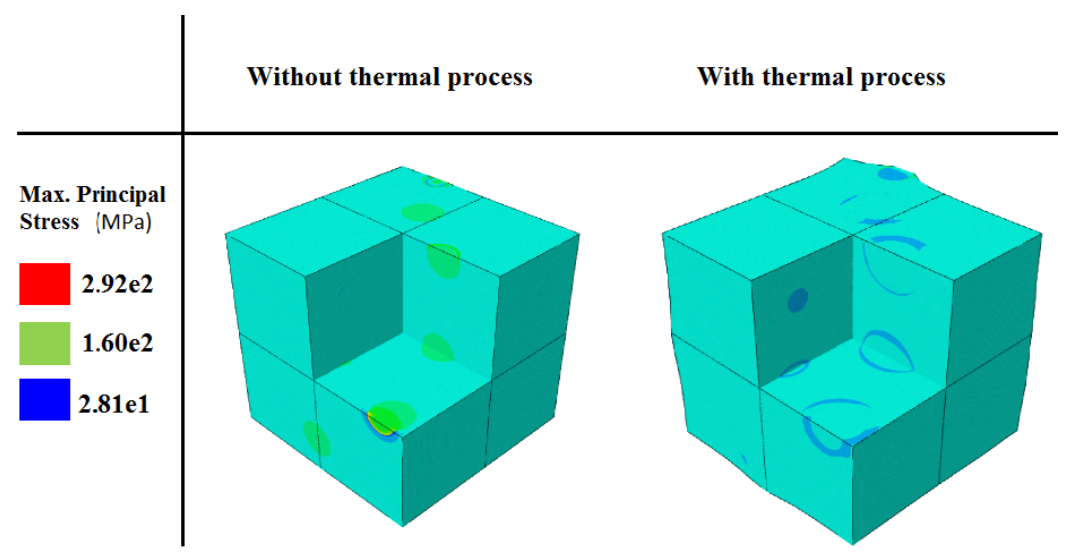

(b)

Figure 3.9: Maximum principal stress with and without thermal process. 


\section{A NEW CLASS OF ELASTIC AND VISCOELASTIC BODIES}

\subsection{Nonlinear elastic model}

The previous section presents a thoroughly analytical and numerical investigation of the prestress effect on the mechanical performance of composites within the context of classical linearized elastic/viscoelastic constitutive models. This chapter studies the problem within the context of a new class of elastic bodies [29, 30, 31], which is a subset of a generalization of classical Cauchy and Green elastic bodies, and then extend the model to quasi-linear viscoelastic model to incorporate the time-dependence effect.

The generalization allows for an elastic body to be defined through:

$$
\mathbf{f}(\mathbf{B}, \boldsymbol{\sigma}, \varrho)=\mathbf{0}
$$

where $\mathbf{B}$ is Cauchy-Green stretch tensor, $\boldsymbol{\sigma}$ is Cauchy stress tensor, and $\varrho$ is the mass density.

A special subclass of the above class of implicit models is the following class of explicit models that provide an expression for B in terms of $\sigma$, namely (see Rajagopal [28])

$$
\mathbf{B}=\mathbf{g}(\boldsymbol{\sigma}, \varrho)
$$

For isotropic bodies, we have

$$
\mathbf{B}=\alpha_{0} \mathbf{I}+\alpha_{1} \boldsymbol{\sigma}+\alpha_{2} \boldsymbol{\sigma}^{2}
$$

where $\alpha_{i}, i=0,1,2$ depend on the principal invariants of $\boldsymbol{\sigma}$ and the density $\varrho$.

Following the standard linearizion procedure by assuming that the displacement gra- 
dient is small for all time, that is,

$$
\max _{\mathbf{x} \in \kappa(B), t \in \mathbb{R}}\left\|\frac{\partial \mathbf{u}}{\partial \mathbf{x}}\right\|=0(\delta) \quad \delta \ll 1
$$

where $\|\cdot\|$ stands for the usual trace norm. It follows that

$$
\mathbf{E}=\boldsymbol{\varepsilon}+\mathbf{0}\left(\delta^{2}\right), \quad \mathbf{e}=\boldsymbol{\varepsilon}+\mathbf{0}\left(\delta^{2}\right), \quad \mathbf{B}=1+2 \boldsymbol{\varepsilon}+\mathbf{0}\left(\delta^{2}\right)
$$

This leads to a relationship between linearized strain $\varepsilon$ and the stress $\sigma$ for isotropic bodies:

$$
\boldsymbol{\varepsilon}=\beta_{0} \mathbf{I}+\beta_{1} \boldsymbol{\sigma}+\beta_{2} \boldsymbol{\sigma}^{2}
$$

where $\beta_{i}, i=0,1,2$ depend on the principal invariants of $\boldsymbol{\sigma}$.

Since the linearized strain $\varepsilon$ is a nonlinear function of the stress $\sigma$, one can pick the functions and parameters to achieve desired material responses according to the experiment data. In one case, one can obtain bounded strains that can be fixed to be as small as one wishes, while the stress is allowed to grow and even become unbounded. Or if we have a material experience stress softening, one can pick functions and parameters to describe such behavior. A nonlinear function taking the form [32] is:

$$
\boldsymbol{\varepsilon}=-\alpha f_{1}(\boldsymbol{\sigma}) \mathbf{I}+\gamma f_{2}(\boldsymbol{\sigma}) \boldsymbol{\sigma}
$$

where $f_{1}$ and $f_{2}$ are functions of the invariants, $I_{1}=\operatorname{trace}(\boldsymbol{\sigma}), I_{2}=\frac{1}{2} \operatorname{trace}\left(\boldsymbol{\sigma}^{2}\right), I_{3}=$ $\frac{1}{3} \operatorname{trace}\left(\boldsymbol{\sigma}^{3}\right)$. In this study, we chose the nonlinear elastic function $f_{1}(\boldsymbol{\sigma})=\exp \left(\beta I_{1}\right)-1$ and $f_{2}(\boldsymbol{\sigma})=\frac{\exp \left(\delta \sqrt{2 I_{2}}\right)-1}{\sqrt{2 I_{2}}+1}$ by observing the nonlinear elastic response of polyoxymethylene (POM) from Muliana et al.[3]. 
By requiring linearity in $\sigma$, the above model reduces to

$$
\boldsymbol{\varepsilon}=-\alpha \beta \operatorname{tr}(\boldsymbol{\sigma}) \mathbf{I}+\delta \gamma \boldsymbol{\sigma}
$$

Compare with the classical linearized elastic model:

$$
\alpha \beta=\frac{-\nu}{E} \quad \delta \gamma=\frac{1+\nu}{E}
$$

In the following discussion, the material parameters for the stress softening model are calibrated from the experimental data of POM. The constitutive theory has been presented recently (see $[11,12,14,15,19])$, and the implications and consequences of these new class of constitutive relations are examined. It is meaningful to perform parametric study for this model. The same model with different material parameters can be used to describe response of materials with limiting strain and stress softening. The response from these models are compared with classical linearized elastic body. The material parameters used are presented in Table 4.1 [3].

Table 4.1: Nonlinear elastic material parameters [3].

\begin{tabular}{|c|c|c|}
\hline Parameters & Limiting stress model & Limiting strain model \\
\hline$\alpha$ & $0.21 \times 10^{-2}$ & $-0.21 \times 10^{-2}$ \\
\hline$\beta$ & $0.037 \frac{1}{\mathrm{MPa}}$ & $-0.037 \frac{1}{\mathrm{MPa}}$ \\
\hline$\gamma$ & $0.74 \times 10^{-2}$ & $-0.74 \times 10^{-2}$ \\
\hline$\delta$ & $0.037 \frac{1}{\mathrm{MPa}}$ & $-0.037 \frac{1}{\mathrm{MPa}}$ \\
\hline
\end{tabular}




\subsubsection{Numerical implementation}

Numerical method is used to obtain an approximate solution for the corresponding stress output. A Newton-Raphson iteration method is used to minimize the error(Residual)

from linearization. The trial(initial) guess of stress is taken as $\boldsymbol{\sigma}^{t,(t r)}=\boldsymbol{\sigma}^{t,(0)}=\boldsymbol{\sigma}^{t-\Delta t}$. The residual vector $\mathbf{R}^{t,(k)}$ is

$$
\begin{aligned}
\mathbf{R}^{t,(k)} & =-\alpha\left[f_{1}\left(\boldsymbol{\sigma}^{t,(k)}\right)-f_{1}\left(\boldsymbol{\sigma}^{t-\Delta t}\right)\right] \mathbf{I} \\
& +\gamma\left[f_{2}\left(\boldsymbol{\sigma}^{t,(k)}\right) \boldsymbol{\sigma}^{t,(k)}-f_{2}\left(\boldsymbol{\sigma}^{t-\triangle t}\right) \boldsymbol{\sigma}^{t-\triangle t}\right],
\end{aligned}
$$

where $\mathrm{k}$ is iteration counter.

Stress at current time is corrected as:

$$
\boldsymbol{\sigma}^{t,(k+1)}=\boldsymbol{\sigma}^{t,(k)}-\left[\frac{\partial \mathbf{R}^{t,(k)}}{\partial \boldsymbol{\sigma}}\right]^{-1} \mathbf{R}^{t,(k)}
$$

and the consistent tangent matrix is:

$$
\begin{gathered}
\frac{\partial \mathbf{R}^{t,(k)}}{\partial \boldsymbol{\sigma}}=-\alpha \frac{\partial f_{1}\left(\boldsymbol{\sigma}^{t,(k)}\right)}{\partial \boldsymbol{\sigma}} \mathbf{I}+\gamma \frac{\partial f_{2}\left(\boldsymbol{\sigma}^{t,(k)}\right)}{\partial \boldsymbol{\sigma}} \boldsymbol{\sigma}^{t,(k)}+\gamma f_{2}\left(\boldsymbol{\sigma}^{t,(k)}\right) \mathbf{I} \\
\frac{\partial \mathbf{R}^{t,(k)}}{\partial \boldsymbol{\sigma}}=-\alpha \frac{\partial f_{1}\left(\boldsymbol{\sigma}^{t,(k)}\right)}{\partial I_{1}} \mathbf{I}_{A}+\gamma \frac{\partial f_{2}\left(\boldsymbol{\sigma}^{t,(k)}\right)}{\partial I_{2}} \boldsymbol{\Sigma}+\gamma f_{2}\left(\boldsymbol{\sigma}^{t,(k)}\right) \mathbf{I}_{B} \\
\mathbf{I}_{A}=\left[\begin{array}{cccccc}
1 & 1 & 1 & 0 & 0 & 0 \\
1 & 1 & 1 & 0 & 0 & 0 \\
1 & 1 & 1 & 0 & 0 & 0 \\
0 & 0 & 0 & 0 & 0 & 0 \\
0 & 0 & 0 & 0 & 0 & 0 \\
0 & 0 & 0 & 0 & 0 & 0
\end{array}\right] ; \boldsymbol{\Sigma}=\left[\begin{array}{c}
\sigma_{11} \\
\sigma_{22} \\
\sigma_{33} \\
\sigma_{12} \\
\sigma_{13} \\
\sigma_{23}
\end{array}\right]\left[\begin{array}{l}
\sigma_{11} \sigma_{22} \sigma_{33} 2 \sigma_{12} 2 \sigma_{13} 2 \sigma_{23}
\end{array}\right] ; \mathbf{I}_{B}=\mathbf{I}_{6 \times 6}
\end{gathered}
$$




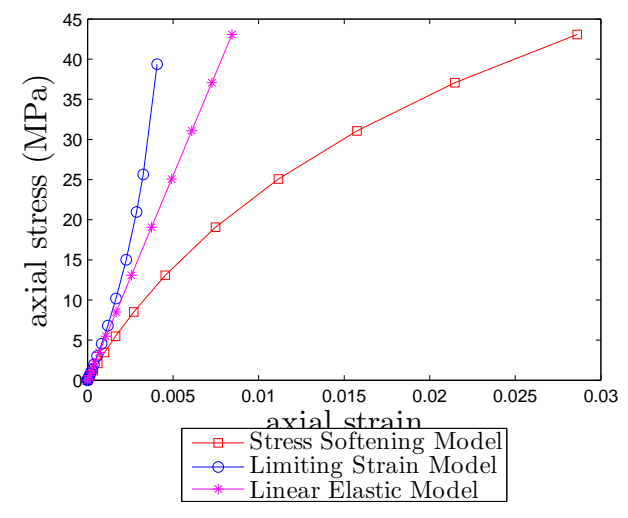

(a) $\sigma_{11}$ vs. $\varepsilon_{11}$.

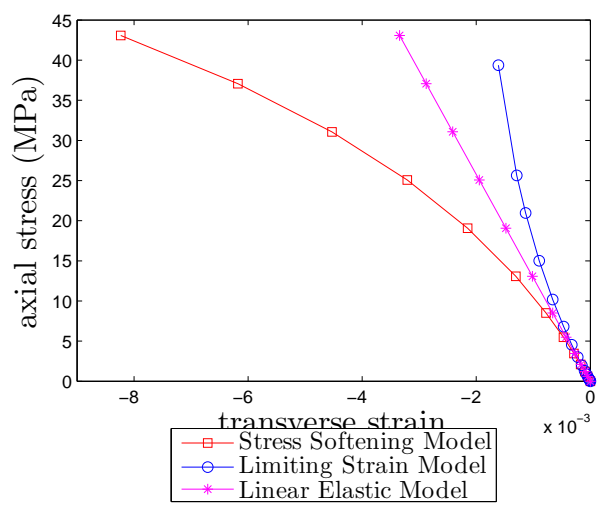

(b) $\sigma_{11} \mathrm{vs} . \varepsilon_{22}$.

Figure 4.1: Uniaxial response for one element with various models in comparison with linear elastic model.

In order to visualize the response of various models, we implemented the user subroutine with one element under uniaxial loading. Fig. 4.1 shows the corresponding stressstrain responses for these models comparing with classical linearized elastic model. For the limiting strain model, the linearized strain remains small even with large stress input, and the stress will approach an asymptotic value for the stress softening model. For all these models we studied, a positive Poisson's ratio are assumed.

\subsubsection{Boundary value problem studies}

The same boundary value problems are considered as in previous sections but now the matrix behaves as in Eqn. 4.7. The parameters for the matrix are listed in Table 4.1 and the inclusion constituent is listed in Table 4.2. 


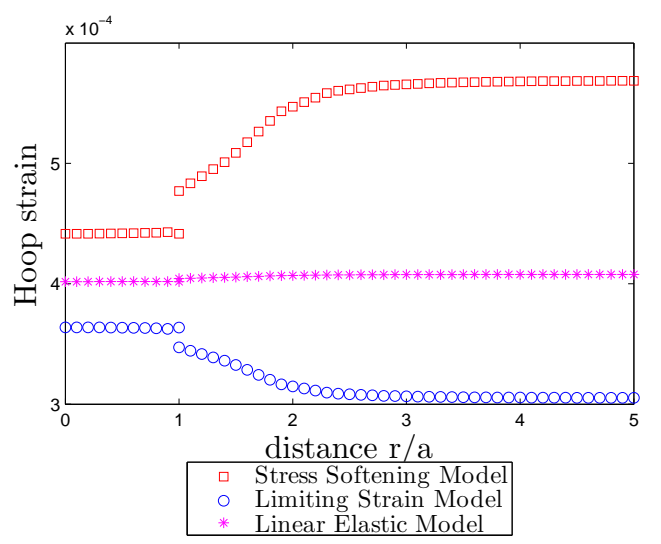

(a) Triaxial loading.

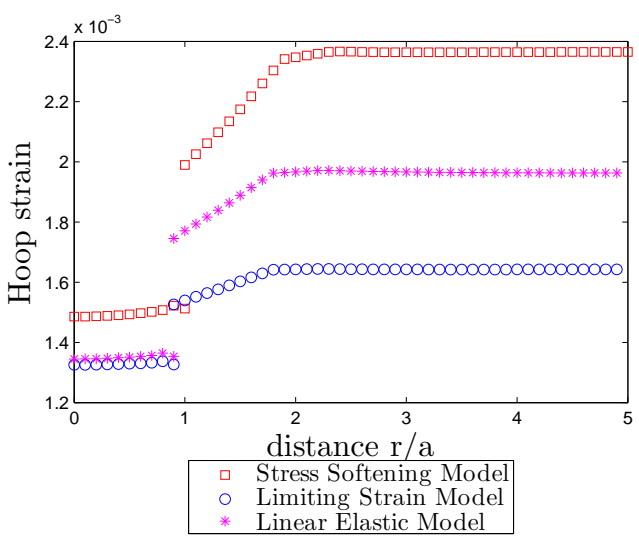

(c) Uniaxial loading.

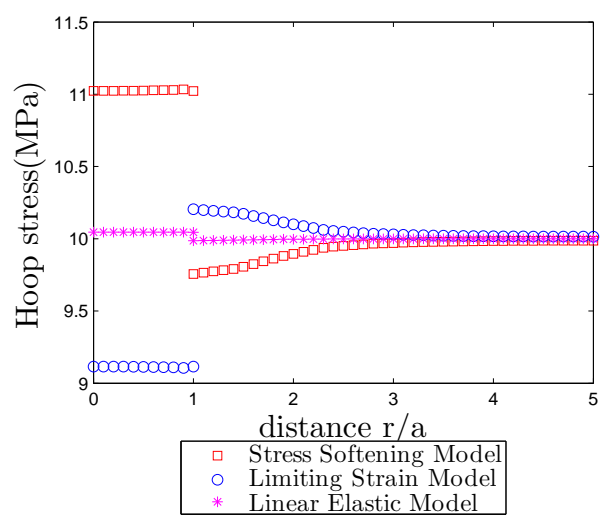

(b) Triaxial loading.

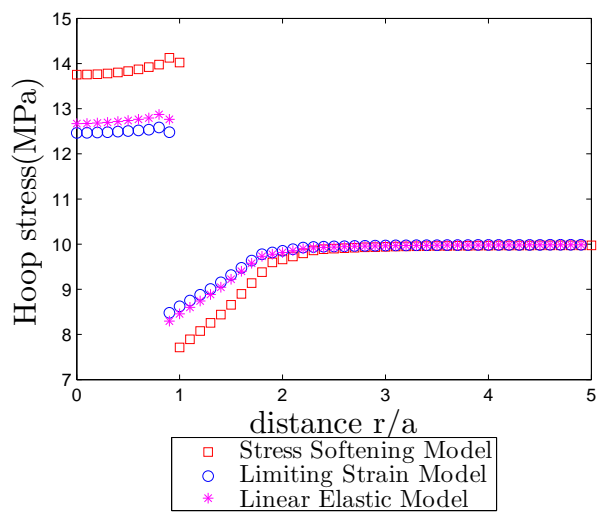

(d) Uniaxial loading.

Figure 4.2: Hoop stresses and strains for composites with one stiffer inclusion.

Table 4.2: Elastic parameters for inclusion.

\begin{tabular}{|c|c|c|}
\hline Parameters & Stiffer inclusion & Softer inclusion \\
\hline$E$ & $10 \mathrm{GPa}$ & $1 \mathrm{GPa}$ \\
\hline$\nu$ & 0.3 & 0.3 \\
\hline
\end{tabular}




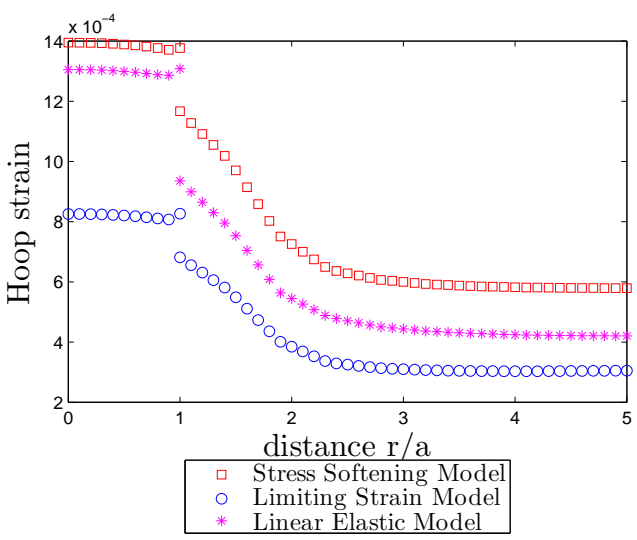

(a) Triaxial loading.

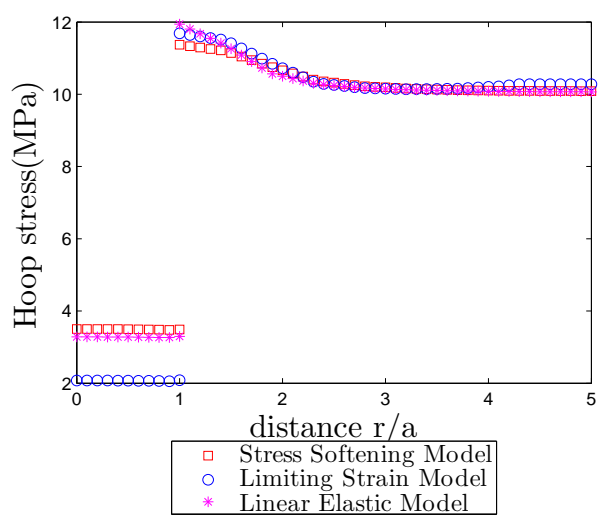

(b) Triaxial loading.

Figure 4.3: Hoop stresses and strains for composite with a softer inclusion.

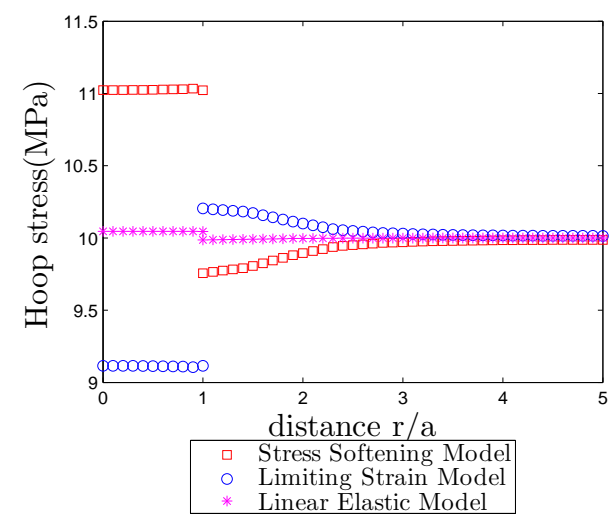

(a) Without prestress.

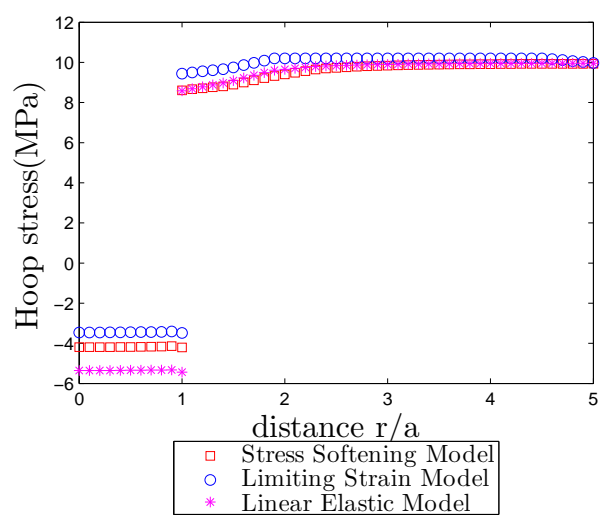

(b) With prestress.

Figure 4.4: Hoop stresses with and without prestress for various nonlinear elastic models in comparison with linear elastic model. 


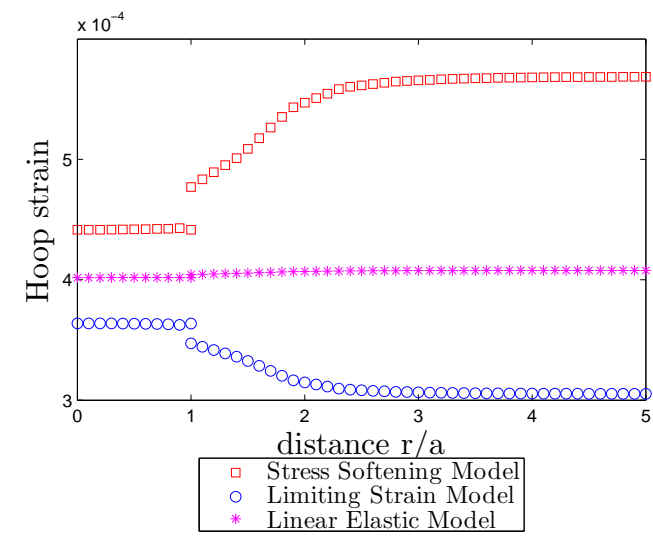

(a) Without prestress.

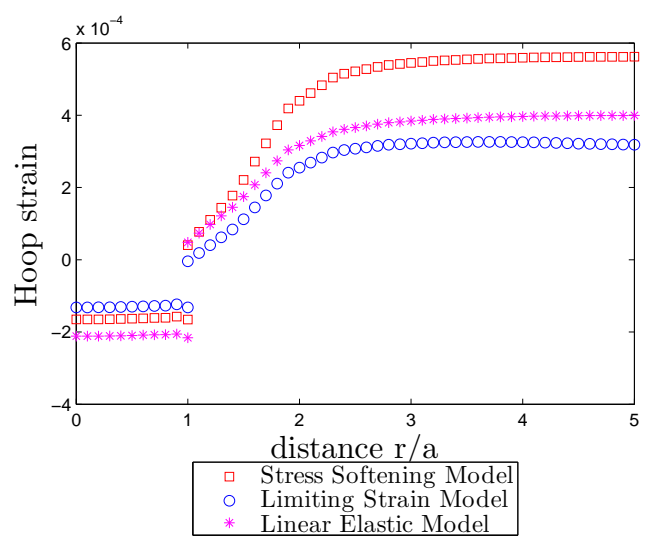

(b) With prestress.

Figure 4.5: Hoop strains with and without prestress for various nonlinear elastic models in comparison with linear elastic model.

Consider first composites with just one linearized elastic inclusion embedded in a elastic medium exhibiting limiting strain or stress softening behavior. Fig. 4.2 and Fig. 4.3 illustrates the hoop stress and hoop strain along the radial direction. The results of linearized elastic matrix are also plotted for comparison. We consider composites with both soft inclusions and brittle inclusions. The composites may resist uniform tensile loading or under uniaxial loading. It is worth noting that for the stress softening model, the relatively small stress in the matrix leads to a higher stress in the inclusion as expected, due to imposing equilibrium condition. For the matrix showing limiting strain behavior, the result agrees with the response presented in [49] and [50], the linearized strain is bounded. Also, it is obvious that with a stiffer inclusion, the stress fields in the inclusion are higher compared to the one with a soft inclusion. Since for composite having an inclusion with a higher elastic modulus, the inclusion will bear more loading.

The hoop stress and strain fields are also plotted for cases with and without prestressing the inclusions. Both nonlinear elastic models are considered and compared with the linear 
elastic case. As illustrated in Fig. 4.4 and 4.5, the compressive prestress on the inclusion reduces the stress fields in the inclusion and with enough prestress value, the tensile stress in the inclusion can be decreased to a compressive stress. For composite with just one inclusion, one may conclude that the deformation in the inclusion reduces significantly while the effect to the matrix is relatively small by applying compressive prestress on the inclusion.

As discussed earlier, composites with $10 \%$ volume fraction under triaxial tensile loading are also evaluated here. The uniform tensile traction prescribed on the boundary is $10 \mathrm{MPa}$ and the compressive prestress on the inclusion is $20 \mathrm{MPa}$. The geometry of the composite is shown in Fig. 3.3a. Fig. 4.6 shows the maximum principal stress and strain with various matrix behaviors with and without prestress. As expected, for stress softening matrix, the stress in the matrix is bounded and the stress in the inclusion is higher compared to the linearized elastic matrix case due to equilibrium requirement. For limiting strain model, the linearized strain is bounded in the matrix. It is also seen that the stress and strain are reduced in the inclusion while the effect to the matrix is negligible with and without prestressing the inclusion. 


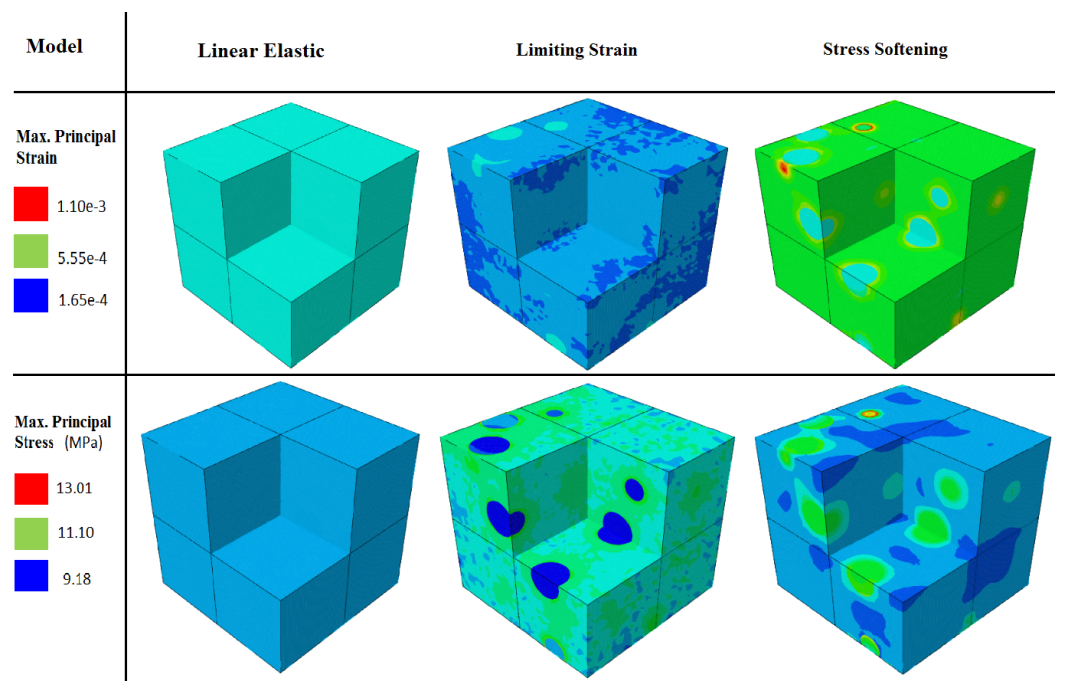

(a) Without prestress.

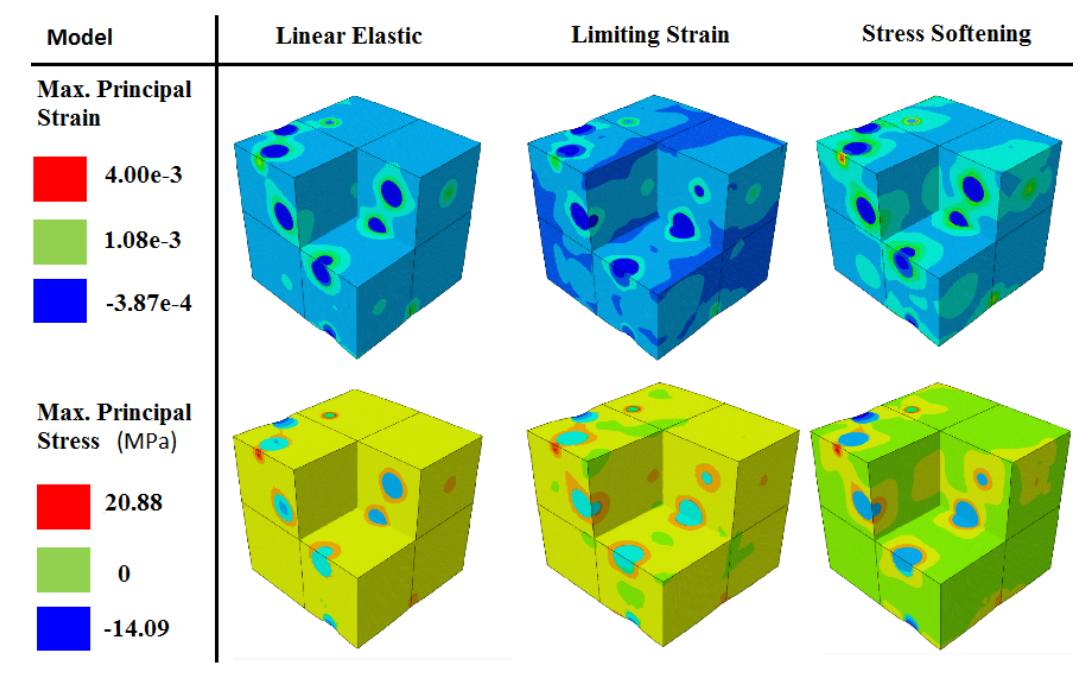

(b) With prestress.

Figure 4.6: Maximum principal stress and strain with and without prestress. 


\subsection{Nonlinear viscoelastic model}

In classical linearized elasticity, one could express stress in terms of the linearized strain and the linearized strain in terms of the stress. Both of these expressions are considered equivalent. As discussed earlier, since force is the cause for deformation, it makes remarkable sense to express the strain in terms of the stress. A similar argument presents itself in linear viscoelasticity wherein one could describe the strain in terms of the stress history instead of expressing the stress in terms of the history of the linearized strain. In analogy to the quasi-linear viscoelastic model developed by Rivlin [51] and others where the stress being given as the function of the kinematic quantity, the new class of quasilinear model we are going to discuss to describe the nonlinear viscoelastic response is [32]

$$
\mathbf{B}=\mathbf{f}(\boldsymbol{\sigma}(0), t)+\int_{0}^{t} \frac{\partial \mathbf{f}(\boldsymbol{\sigma}(s), t-s)}{\partial \boldsymbol{\sigma}(s)} \frac{d \boldsymbol{\sigma}(s)}{d s} d s
$$

where $\mathbf{f}$ is an isotropic function and $\mathbf{B}$ is the Cauchy-Green tensor.

Following the standard linearization procedure as we discussed in the elastic case, the linearized strain $\varepsilon$ is expressed as

$$
\boldsymbol{\varepsilon}=\hat{\mathbf{f}}(\boldsymbol{\sigma}(0), t)+\int_{0}^{t} \frac{\partial \hat{\mathbf{f}}(\boldsymbol{\sigma}(s), t-s)}{\partial \boldsymbol{\sigma}(s)} \frac{d \boldsymbol{\sigma}(s)}{d s} d s
$$

where $\hat{\mathbf{f}}$ is $\frac{1}{2}[\mathbf{f}-\mathbf{I}]$.

It is also worth noting that the way of expressing strain as a function of stress and its history not only in accordance with the cause and effect argument, but also makes more natural sense from experimental viewpoint. Creep tests are natural and straightforward to be carried out for viscoelastic bodies, and for stress relaxation experiments a more complicated apparatus is involved. Muliana et al. [32] has discussed a new class of quasi- 
linear model within one dimensional study. Following from the constitutive relation (4.14)

$$
\hat{f}(\sigma(t), t)=G(\sigma) J(t)
$$

where the function $G(\sigma)$ is a nonlinear function of the stress and $J(t)$ is the creep function. When $G(\sigma)$ is a linear function of the stress, this model can reduce to the constitutive relation for a linear viscoelastic body. For materials with fading memory, the creep function $J(t)$ could take the form

$$
J(t)=J(0)+\sum_{n=1}^{N} J_{n}\left(1-\exp \left[-\lambda_{n} t\right]\right)
$$

The nonlinear function $G(\sigma)$ is analogy to the strain function discussed in the previous section for the nonlinear elastic bodies.

Now, in analogy to linear elastic case, we extend the one dimensional model to three dimensional model by assuming two time dependent creep function $B(t)$ and $J(t)$ :

$$
\begin{aligned}
B(t) & =B(0)+\sum_{n=1}^{N} B_{n}\left(1-\exp \left[-\tau_{n} t\right]\right) \\
J(t) & =J(0)+\sum_{n=1}^{N} J_{n}\left(1-\exp \left[-\lambda_{n} t\right]\right)
\end{aligned}
$$

Following from Eqn. 4.7, the three dimensional nonlinear viscoelastic model takes the form:

$$
\boldsymbol{\varepsilon}=-\alpha \int_{0}^{t} B(t-s) \frac{d\left(f_{1}(\boldsymbol{\sigma}) \operatorname{tr}(\boldsymbol{\sigma})\right)}{d s} d s \quad \mathbf{I}+\gamma \int_{0}^{t} J(t-s) \frac{d\left(f_{2}(\boldsymbol{\sigma}) \boldsymbol{\sigma}\right)}{d s} d s
$$

Consider the same $f_{1}$ and $f_{2}$ functions as in nonlinear elastic case and the time depen- 
dent functions $B_{n}$ and $J_{n}$ are listed in Table 4.3[3].

Table 4.3: Time dependent functions [3].

\begin{tabular}{|c|c|c|c|}
\hline $\mathrm{n}$ & $\tau_{n}=\lambda_{n}$ & $B_{n}$ & $J_{n}$ \\
\hline 0 & - & 1.0 & 1.0 \\
1 & 5 & 0.017 & 0.015 \\
2 & 10 & 0.025 & 0.02 \\
3 & 100 & 0.03 & 0.03 \\
4 & 500 & 0.03 & 0.05 \\
5 & 1000 & 0.1 & 0.08 \\
6 & 5000 & 0.16 & 0.15 \\
7 & 10000 & 0.3 & 0.2 \\
8 & 50000 & 0.54 & 0.5 \\
\hline
\end{tabular}

This new class of quasi-linear viscoelastic model will be implemented and with the FE formulation, the analysis within the context of the composites will be discussed. We will discuss the problem within the context of several constitutive theories and make contrast to the results for the classical linearized elastic and viscoelastic bodies. 


\subsubsection{Numerical implementation}

Following from Eqn. 4.19, the convolution integral is divided into recursive parts. we have

$$
\begin{aligned}
\varepsilon_{i j}^{t} & =-\left[\alpha B(0) f^{t} \sigma_{k k}^{t}-\alpha \Sigma B_{n} f^{t} \sigma_{k k}^{t}+\alpha \Sigma B_{n} \int_{0}^{t} \exp \left[-(t-s) / \tau_{n}\right] \frac{d\left(f_{1} \sigma_{k k}\right)}{d s} d s\right] \\
& \delta_{i j}+\gamma J(0) f^{t} \sigma_{i j}^{t}+\gamma \Sigma J_{n} f^{t} \sigma_{i j}^{t}-\gamma \Sigma J_{n} \int_{0}^{t} \exp \left[-(t-s) / \lambda_{n}\right] \frac{d\left(f_{1} \sigma_{i j}\right)}{d s} d s \\
& =-\alpha\left(B(0)+\Sigma B_{n}\right) f^{t} \sigma_{k k}^{t} \delta_{i j}+\alpha \Sigma B_{n} q_{n}^{t} \delta_{i j} \\
& +\gamma\left(J(0)+\Sigma J_{n}\right) f^{t} \sigma_{i j}^{t}-\gamma \Sigma J_{n} p_{i j, n}^{t}
\end{aligned}
$$

where $q_{n}^{t}$ and $p_{i j, n}^{t}$ are state variables taking the following forms:

$$
\begin{aligned}
q_{n}^{t} & =e^{-\Delta t / \tau_{n}} q_{n}^{t-\Delta t}+\int_{t-\Delta t}^{t} \exp \left[-(t-s) / \tau_{n}\right] \frac{d\left(f_{1} \sigma_{k k}\right)}{d s} d s \\
& =e^{-\Delta t / \tau_{n}} q_{n}^{t-\Delta t}+\frac{\Delta t}{2}\left[\frac{f_{1}^{t} \sigma_{k k}^{t}-f_{1}^{t-\Delta t} \sigma_{k k}^{t-\Delta t}}{\Delta t}+\left.e^{-\Delta t / \tau_{n}} \frac{\Delta\left(f_{1} \sigma_{k k}\right)}{\Delta t}\right|_{t-\Delta t}\right] \\
p_{i j, n}^{t} & =e^{-\Delta t / \lambda_{n}} p_{i j, n}^{t-\Delta t}+\int_{t-\Delta t}^{t} \exp \left[-(t-s) / \lambda_{n}\right] \frac{d\left(f_{2} \sigma_{i j}\right)}{d s} d s \\
& =e^{-\Delta t / \lambda_{n}} p_{i j, n}^{t-\Delta t}+\frac{\Delta t}{2}\left[\frac{f_{2}^{t} \sigma_{i j}^{t}-f_{2}^{t-\Delta t} \sigma_{i j}^{t-\Delta t}}{\Delta t}+\left.e^{-\Delta t / \lambda_{n}} \frac{\Delta\left(f_{2} \sigma_{i j}\right)}{\Delta t}\right|_{t-\Delta t}\right]
\end{aligned}
$$

The recursive approach minimizes the storage required to perform the constitutive integration. Using Newton-Raphson iteration method to minimize the error from linearizion. As we discussed in the nonlinear elastic section, using the trial guess stress $\boldsymbol{\sigma}^{t,(t r)}=\boldsymbol{\sigma}^{t,(0)}=$ $\boldsymbol{\sigma}^{t-\triangle t}$, we can update the stress and tangent stiffness matrix when the residual error falls into the desired tolerance. Once the convergence is achieved, the hereditary integrals in each prony series are calculated and stored in order to be used in the next time integration step. Detailed derivatives of the recursive-iterative algorithm can be found in APPENDIX. Table. 4.4 illustrates the user material subroutine algorithm. 
Table 4.4: Recursive-iterative algorithm.

1.Input variables: (at the $m^{\text {th }}$ global iteration)

$\varepsilon_{i j}^{t-\triangle t}, \triangle \varepsilon_{i j}^{t-\triangle t,(m)}, \triangle t^{(m)} \quad$ History $\sigma_{i j}^{t-\Delta t}, p_{i j, n}^{t-\Delta t}, q_{n}^{t-\triangle t}$

2.Initial approximation variables:

$$
\sigma_{i j}^{t,(0)}=\sigma_{i j}^{t-\triangle t}
$$

3.Iterate for $k=1,2,3 \ldots$ ( $\mathrm{k}$ is local iteration counter)

- Compute stress correction

$\sigma_{i j}^{t,(k+1)}=\sigma_{i j}^{t,(k)}-\left[\frac{\partial R_{i j}^{t,(k)}}{\partial \sigma_{k l}^{t}}\right]^{-1} R_{k l}^{t,(k)}$

$\checkmark$ Evaluate residual tensor

$R_{i j}^{t,(k+1)}=e_{i j}^{t,(k+1)}+\frac{1}{3} \varepsilon_{k k}^{t,(k+1)}-\hat{\varepsilon}_{i j}^{t,(m)}$

IF $\left\|R_{i j}^{t,(k+1)}\right\| \leq$ Tol THEN GOTO 4 and EXIT

\section{ENDIF GOTO 3}

4. Update stress, consistent tangent stiffness, and history variables:

$\sigma^{t} \leftarrow \sigma^{t,(k+1)} \quad C_{i j k l}^{t} \quad p_{i j, n}^{t} \leftarrow p_{i j, n}^{t,(k+1)} \quad q_{n}^{t} \leftarrow q_{n}^{t,(k+1)}$

The numerical constitutive model is then implemented within a displacement based FE structural analysis environment. The user material subroutine is implemented with commercial software ABAQUS to perform parametric studies on composites with various constituents.

We considered two nonlinear viscoelastic models: one with the limiting strain behavior and the other one with stress softening behavior. The user subroutines are implemented with one element under uniaxial loading to visualize the creep response for these viscous models. Using the same nonlinear elastic parameters as we used in the previous section, the corresponding stress-strain responses are plotted in comparison with the linear vis- 
coelastic response.

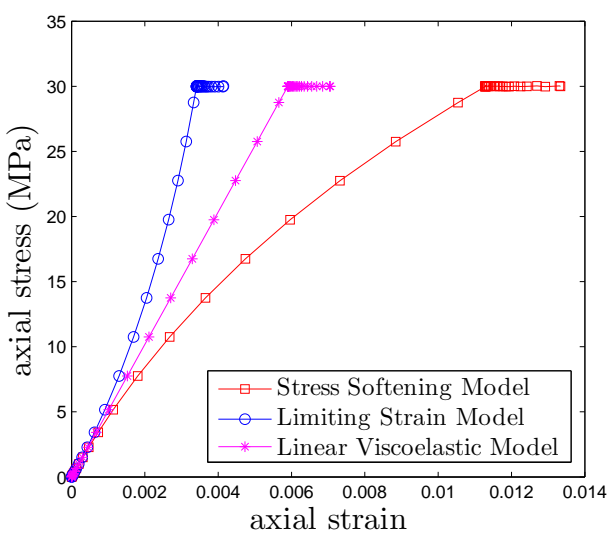

(a) $\sigma_{11} \mathrm{vs} \cdot \varepsilon_{11}$.

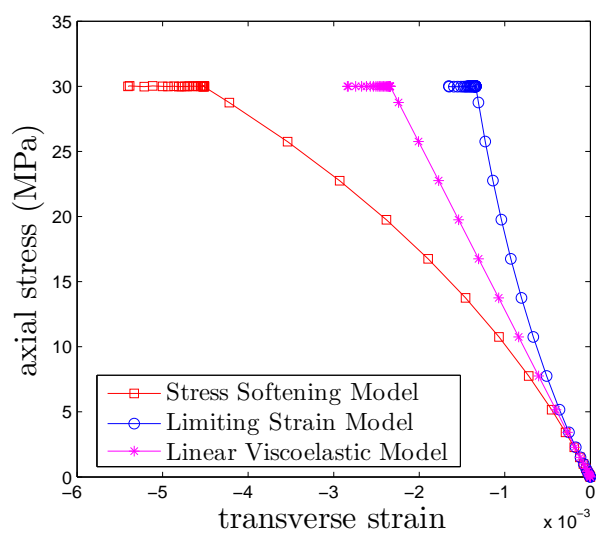

(b) $\sigma_{11} \mathrm{vs} . \varepsilon_{22}$.

Figure 4.7: Uniaxial response for one element for nonlinear viscoelastic models in comparison with linear viscoelastic model.

Creep can be observed from Fig. 4.7 for three viscous models. We assume positive Poison's ratio for all three models. Still, comparing to linear viscoelastic model, the linearized strain remains small even with large stress input for the limiting strain model and the stress will approach asymptotic value for the stress softening model. Despite of the various nonlinear instantaneously elastic behaviour, as time increase, these models show clear creep which is as expected.

\subsubsection{Boundary value problem studies}

With the user subroutine in hand, let's perform parametric studies for composites with various volume fractions.

Consider composite with only one inclusion and the matrix shows nonlinear viscoelastic behaviour as we described in Eqn. 4.19. The parameters for the matrix are listed in 
Table 4.1 for the elastic parts and Table 4.3 for the time dependent creep parts and the inclusion constituent is listed in Table 4.2. Fig. 4.8a depicts the hoop strain dependence on time without prestress for stress softening model while Fig. $4.8 \mathrm{~b}$ shows the hoop strain fields with prestress. Creep behavior can be noted clearly and also the transfer of tensile stress to compressive stress in the inclusion. Fig. 4.9 shows the hoop strain with respect to time with and without prestress for limiting strain nonlinear viscoelastic model. Since matrix still has viscous part, creep response can also be seen here. It is also noted that the strain in the matrix modeled with limiting strain model shows a limited value compared to the one modeled with stress softening model.

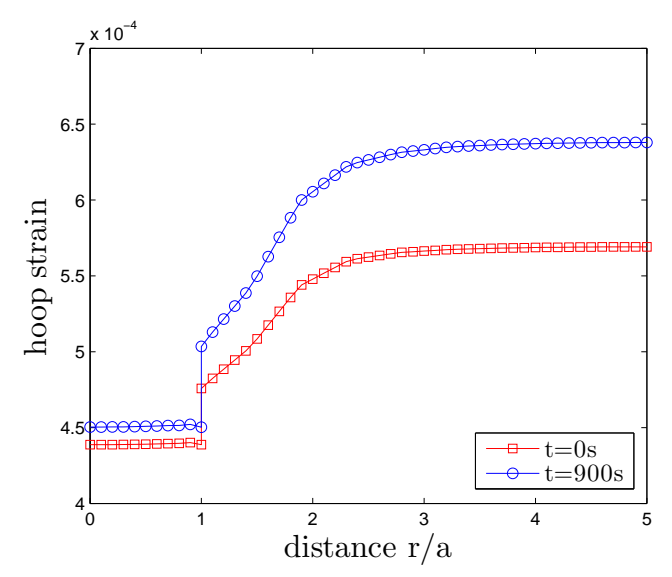

(a) Without prestress.

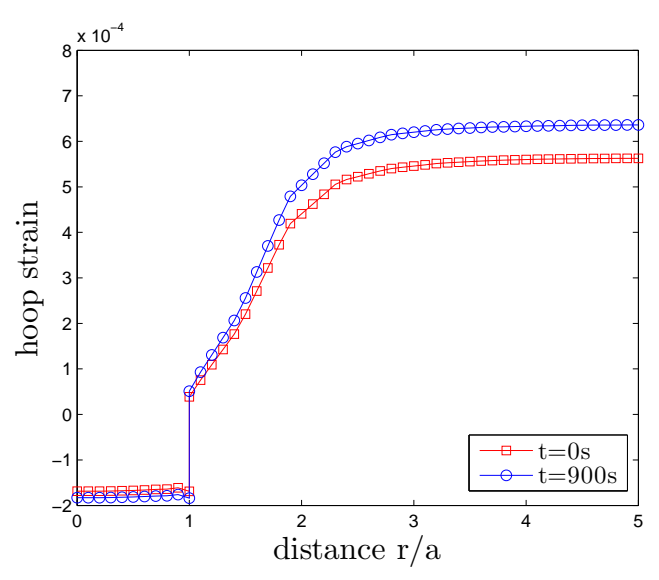

(b) with prestress.

Figure 4.8: Hoop strain dependence on time for nonlinear viscoelastic model showing stress softening behavior. 


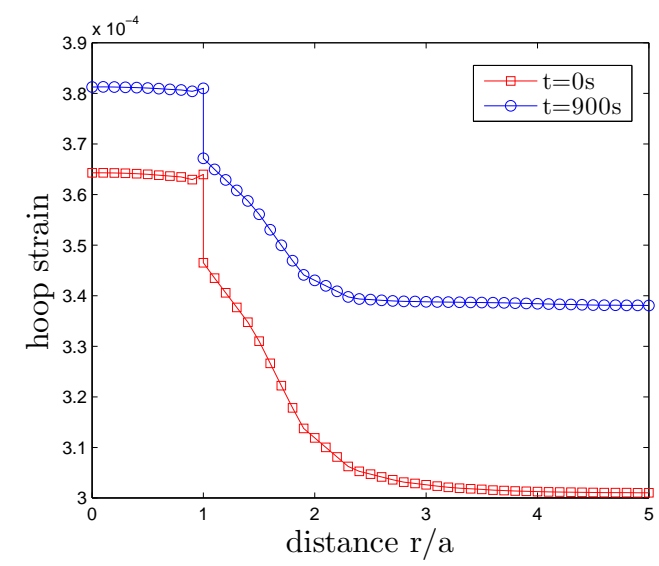

(a) Without prestress.

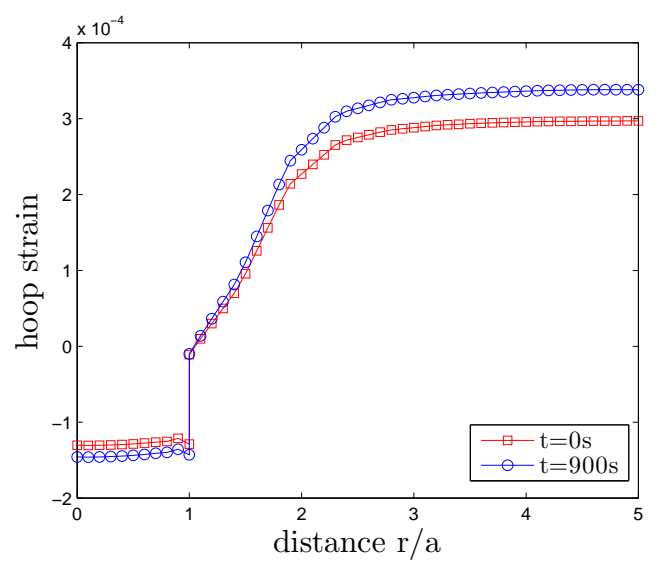

(b) with prestress.

Figure 4.9: Hoop strain dependence on time for nonlinear viscoelastic model showing limiting strain behavior.

Composites with $10 \%$ particle volume content under triaxial tensile loading are also discussed here. The geometry of the composite is already discussed before as in Fig. 3.3a. Fig. 4.10 depicts the maximum principal stress and strain with and without prestress for composite comprise of matrix modeled with stress softening model. It is noted that the prestress on the inclusion transfer the tensile stress to compressive stress for the inclusion. Fig. 4.11 shows the maximum principal strain with respect to time for composite having matrix with stress softening behavior. The two plots depict the strain field at early time and later time during creep respectively. It is obvious that the matrix which is modeled as viscoelastic material experience creep as expected. Similarly, creep response can also be observed from Fig. 4.12 which shows the strain response for composite comprise of matrix having limiting strain behavior. Fig. 4.13 compares the axial displacement responses for composites comprise of various properties of matrix with and without prestress. The axial displacement is the least for the matrix showing limiting strain behavior which is as expected. Since the creep functions are choosing to be the same for all these models, 
the creep responses are very similar for all of them. The overall deformation response decrease due to the compressive prestress in the inclusion.

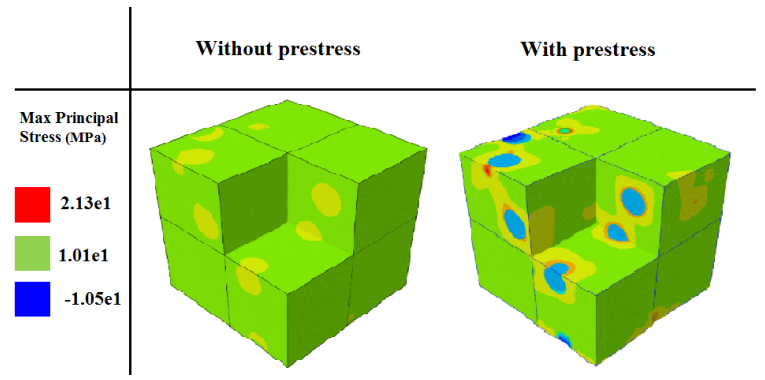

(a) Maximum principal stress.

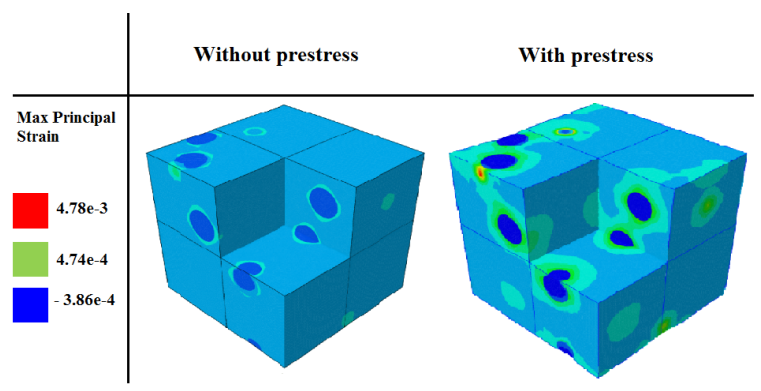

(b) Maximum principal strain.

Figure 4.10: Maximum principal stress and strain with and without prestress.

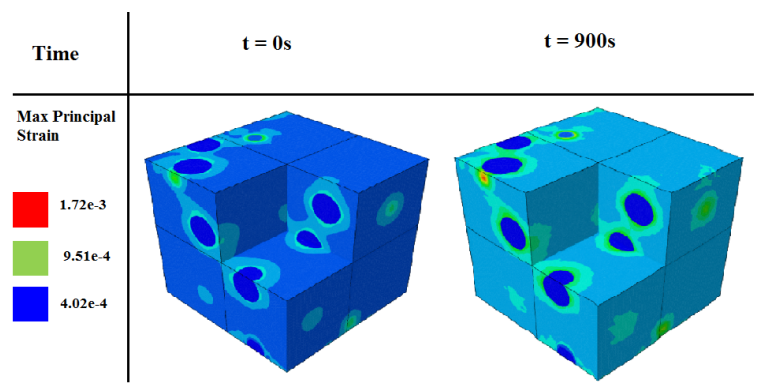

Figure 4.11: Maximum principal strain dependence on time with matrix showing stress softening behavior. 


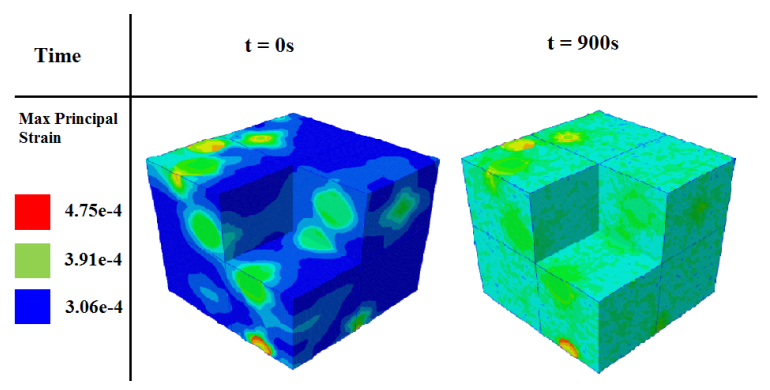

Figure 4.12: Maximum principal strain dependence on time with matrix showing limiting strain behavior.

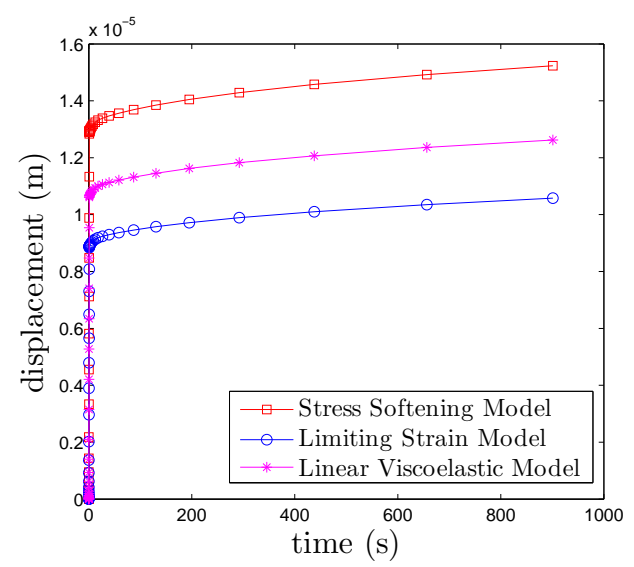

(a) Without prestress.

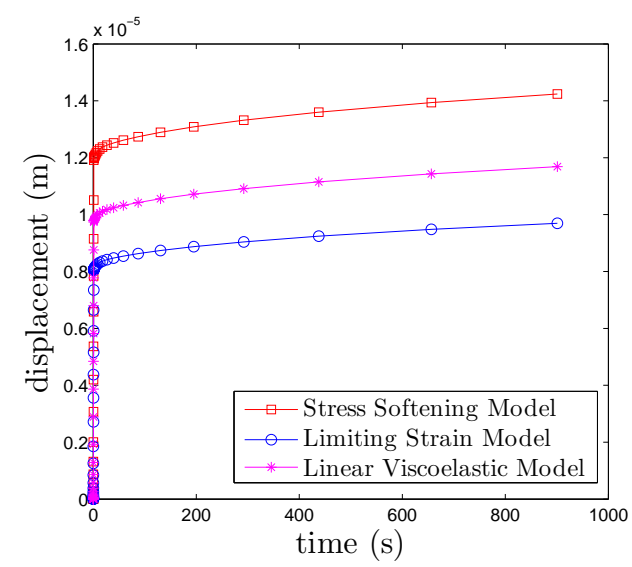

(b) With prestress.

Figure 4.13: Axial displacement for three viscoelastic models. 


\section{SUMMARY}

In this dissertation, a novel theoretical and computational study of the possibility of enhancing the overall performance of composites by pre-stressing one or more of the constituents of a composite has been presented.

When composites comprising of brittle inclusions and ductile/soft matrix are considered, high values in the hoop stress discontinuities are observed. These high stress discontinuities can lead to debonding between the inclusion and matrix, reducing the load carrying capacity of the composites. It is also observed that for the above composites under uniform external tensile load, the magnitude of the tensile stress in the brittle inclusion and in the matrix decreases significantly by introducing the compressive prestress to the brittle inclusions. Reducing the tensile stresses in the brittle inclusion and ductile matrix can delay or minimize failure in the constituents due to cracking. Furthermore, the stiffness of the constituents also influences the magnitude of stresses in the composites. As expected the instantaneous modulus of the viscoelastic matrix only affects the response at early time. The softer the matrix, the smaller the stress discontinuity at the interface is. Thus, when a brittle and stiffer inclusion is used for composites that are designed to sustain external uniform tensile stresses, applying compressive prestress in the inclusion and considering softer or compliant matrix hold promise with regard to enhancing load carrying capacity of composites. Unlike the case of uniform external tensile stress, when the composite is subjected to a uniaxial tensile load the hoop stress discontinuities at the interface between the inclusion and matrix do not necessarily drop with increasing the compressive prestress. At certain locations, the stress discontinuity is small, whereas at other locations the stress discontinuity increases. Although in the case of uniaxial loading, the stress discontinuities would vary at different locations and prestressing the inclusion 
does not necessarily reduce the entire stress discontinuities, certain choice of non-uniform prestressing can still give an enhanced performance when taking into account the overall response of the composites.

FE analyses of composites due to prestress are presented by incorporating more realistic microstructures, but still within certain simplification and approximation. The composite contains uniform solid spherical inclusions of a brittle material, each of radius $0.001 \mathrm{~mm}$, dispersed randomly in the viscoelastic matrix domain. Prestressing the brittle inclusions induces compressive stresses to the brittle inclusions, while its effect on the stress distributions in the matrix is negligible. Thus, prestressing the inclusions is promising in increasing tensile load carrying capacity in composites comprising of brittle inclusions. In the composite with higher particle contents, there are some localized stresses both in the particles and matrix, which could be due to relatively small distances between particles in the matrix. High localized stresses could induce failure in the composites, reducing the load carrying capacity of the composites. Next, the effect of the properties of interstitial matters on the overall response of composite is studied. The role of the interstitial matters is significant in affecting the overall performance of the composites. When the interstitial matters are stiffer than the particles the matrix experiences higher stresses as compared to the composites with soft interphases. The interstitial matters also influence the amount of the compressive stresses in the brittle particles. Moreover, strains increase with time due to the viscoelastic matrix and continuously increasing strains with time could eventually also lead to delamination between inclusions and matrix.

Also, the FE analysis was conducted so as to examine a possible method to induce prestress on the brittle inclusions, coated by soft polymer interphases, by temperature changes. When a processing method involves curing at an elevated temperature, upon cooling down to room temperature, the mismatches in the thermal and mechanical properties of the inclusions, interphases, and matrix induce compressive prestresses in the in- 
clusions, while the soft interphases experience tension and the matrix is under nearly zero stresses. It is also observed that the shear stresses in the constituents are negligible. This study shows that prestressing brittle inclusions could lead to increasing load carrying capacity of composites under tensile loading.

Finally, composites comprise of constituents showing nonlinear elastic or nonlinear viscoelastic behavior which fall into a new calss of material models are evaluated. The linearized strain remains small even with large stress input for limiting strain model and the stress will approach an asymptotic value for the stress softening model. The prestressing on the inclusion still affects the stress fields in the inclusion while the influence on the matrix is negligible. 


\section{REFERENCES}

[1] H. Zhu, A. Muliana, and K. Rajagopal, "Effect of prestress on the mechanical performance of composites," Journal of Engineering Mechanics, p. 04015011, 2015.

[2] A. Muliana and K. A. Khan, "A time-integration algorithm for thermo-rheologically complex polymers," Computational Materials Science, vol. 41, no. 4, pp. 576-588, 2008.

[3] A. Muliana, K. Rajagopal, and D. Tscharnuter, "A nonlinear integral model for describing responses of viscoelastic solids," International Journal of Solids and Structures, vol. 58, pp. 146-156, 2015.

[4] G. D. Mancarti, "Strengthening California steel bridges by prestressing," Transportation research record, vol. 950, pp. 183-7, 1984.

[5] M. Tochacek and C. L. Mehta, "Economical design of presstressed plate girder," Journal of the Structural Division, vol. 98, no. 6, pp. 1273-1289, 1972.

[6] F. Klaiber and B. Beck, "Strengthening of existing single span steel beam and concrete deck bridges," System, vol. 2, p. 3, 1985.

[7] H. Saadatmanesh, P. Albrecht, and B. M. Ayyub, "Experimental study of prestressed composite beams," Journal of Structural Engineering, vol. 115, no. 9, pp. 2348$2363,1989$.

[8] H. Saadatmanesh, P. Albrecht, and B. M. Ayyub, "Analytical study of prestressed composite beams," Journal of Structural Engineering, vol. 115, no. 9, pp. 2364 $2381,1989$. 
[9] B. M. Ayyub, Y. G. Sohn, and H. Saadatmanesh, "Prestressed composite girders. i: Experimental study for negative moment," Journal of Structural Engineering, vol. 118, no. 10, pp. 2743-2762, 1992.

[10] J. Nie, C. Cai, T. Zhou, and Y. Li, "Experimental and analytical study of prestressed steel-concrete composite beams considering slip effect," Journal of Structural Engineering, vol. 133, no. 4, pp. 530-540, 2007.

[11] P. G. Hoadley, "Behavior of prestressed composite steel beams," Journal of the Structural Division, vol. 89, no. 3, pp. 21-34, 1963.

[12] R. S. Reagan and N. W. Krahl, "Behavior of prestressed composite beams," Journal of the Structural Division, vol. 93, no. 6, pp. 87-108, 1967.

[13] L. Dezi, G. Leoni, and A. M. Tarantino, "Time-dependent analysis of prestressed composite beams," Journal of Structural Engineering, vol. 121, no. 4, pp. 621-633, 1995.

[14] R. Wood, S. Avadhanula, R. Sahai, E. Steltz, and R. Fearing, "Microrobot design using fiber reinforced composites," Journal of Mechanical Design, vol. 130, p. 052304 , 2008.

[15] T. C. Triantafillou and N. Deskovic, "Innovative prestressing with frp sheets: mechanics of short-term behavior," Journal of Engineering Mechanics, vol. 117, no. 7, pp. 1652-1672, 1991.

[16] G. J. Dvorak and P. Prochazka, "Thick-walled composite cylinders with optimal fiber prestress," Composites Part B: Engineering, vol. 27, no. 6, pp. 643-649, 1996.

[17] M. Tuttle, R. Koehler, and D. Keren, "Controlling thermal stresses in composites by means of fiber prestress," Journal of Composite Materials, vol. 30, no. 4, pp. 486$502,1996$. 
[18] A. P. Suvorov and G. J. Dvorak, "Optimized fiber prestress for reduction of free edge stresses in composite laminates," International Journal of Solids and Structures, vol. 38, no. 38, pp. 6751-6786, 2001.

[19] K. S. Fancey, "Fiber-reinforced polymeric composites with viscoelastically induced prestress," Journal of Advanced Materials, vol. 37, no. 2, pp. 21-29, 2005.

[20] A. Mukherjee and G. L. Rai, "Performance of reinforced concrete beams externally prestressed with fiber composites," Construction and Building Materials, vol. 23, no. 2, pp. 822-828, 2009.

[21] S.-Y. Fu, X.-Q. Feng, B. Lauke, and Y.-W. Mai, "Effects of particle size, particle/matrix interface adhesion and particle loading on mechanical properties of particulate-polymer composites," Composites Part B: Engineering, vol. 39, no. 6, pp. 933-961, 2008.

[22] S. Sinha Ray and M. Okamoto, "Polymer/layered silicate nanocomposites: a review from preparation to processing," Progress in polymer science, vol. 28, no. 11, pp. 1539-1641, 2003.

[23] H.-L. Tyan, Y.-C. Liu, and K.-H. Wei, "Thermally and mechanically enhanced clay/polyimide nanocomposite via reactive organoclay," Chemistry of materials, vol. 11, no. 7, pp. 1942-1947, 1999.

[24] H. Shi, T. Lan, and T. J. Pinnavaia, "Interfacial effects on the reinforcement properties of polymer-organoclay nanocomposites," Chemistry of materials, vol. 8, no. 8, pp. 1584-1587, 1996.

[25] Y.-H. Zhang, J.-T. Wu, S.-Y. Fu, S.-Y. Yang, Y. Li, L. Fan, R. K.-Y. Li, L.-F. Li, and Q. Yan, "Studies on characterization and cryogenic mechanical properties of 
polyimide-layered silicate nanocomposite films," Polymer, vol. 45, no. 22, pp. 75797587, 2004.

[26] J. Cho, M. Joshi, and C. Sun, "Effect of inclusion size on mechanical properties of polymeric composites with micro and nano particles," Composites Science and Technology, vol. 66, no. 13, pp. 1941-1952, 2006.

[27] K.-t. Lau, C. Gu, and D. Hui, "A critical review on nanotube and nanotube/nanoclay related polymer composite materials," Composites Part B: Engineering, vol. 37, no. 6 , pp. 425-436, 2006.

[28] K. R. Rajagopal, "On implicit constitutive theories," Applications of Mathematics, vol. 48, no. 4, pp. 279-319, 2003.

[29] K. Rajagopal, “The elasticity of elasticity," Zeitschrift für angewandte Mathematik und Physik, vol. 58, no. 2, pp. 309-317, 2007.

[30] K. Rajagopal and A. Srinivasa, "On the response of non-dissipative solids," Proceedings of the Royal Society A: Mathematical, Physical and Engineering Science, vol. 463, no. 2078, pp. 357-367, 2007.

[31] K. Rajagopal and A. Srinivasa, "On a class of non-dissipative materials that are not hyperelastic," Proceedings of the Royal Society A: Mathematical, Physical and Engineering Science, vol. 465, no. 2102, pp. 493-500, 2009.

[32] A. Muliana, K. Rajagopal, and A. Wineman, "A new class of quasi-linear models for describing the nonlinear viscoelastic response of materials," Acta Mechanica, vol. 224, no. 9, pp. 2169-2183, 2013.

[33] K. Rajagopal, "Non-linear elastic bodies exhibiting limiting small strain," Mathematics and Mechanics of Solids, vol. 16, no. 1, pp. 122-139, 2011. 
[34] C. O. Horgan and G. Saccomandi, "Phenomenological hyperelastic strain-stiffening constitutive models for rubber," Rubber chemistry and technology, vol. 79, no. 1, pp. 152-169, 2006.

[35] J. D. Ferry, Viscoelastic properties of polymers. John Wiley \& Sons, 1980.

[36] K. R. Rajagopal and A. Wineman, "A useful correspondence principle in the theory of linear materials," Journal of Elasticity, vol. 10, no. 4, pp. 429-434, 1980.

[37] K. Rajagopal and A. Wineman, "A quasi-correspondence principle for quasi-linear viscoelastic solids," Mechanics of Time-Dependent Materials, vol. 12, no. 1, pp. 1$14,2008$.

[38] Z. Bazant, "Matrix differential equation and higher-order numerical methods for problems of non-linear creep, viscoelasticity and elasto-plasticity," Int. J. Numer. Methods Eng, vol. 4, pp. 11-15, 1972.

[39] A. Pipkin and T. Rogers, "A non-linear integral representation for viscoelastic behaviour," Journal of the Mechanics and Physics of Solids, vol. 16, no. 1, pp. 59-72, 1968.

[40] R. L. Taylor, K. S. Pister, and G. L. Goudreau, "Thermomechanical analysis of viscoelastic solids," International Journal for Numerical Methods in Engineering, vol. 2, no. 1, pp. 45-59, 1970.

[41] R. A. Schapery, "On the characterization of nonlinear viscoelastic materials," Polymer Engineering \& Science, vol. 9, no. 4, pp. 295-310, 1969.

[42] J. Lai and A. Bakker, "3-d schapery representation for non-linear viscoelasticity and finite element implementation," Computational Mechanics, vol. 18, no. 3, pp. 182$191,1996$. 
[43] D. Touati and G. Cederbaum, "On the prediction of stress relaxation from known creep of nonlinear materials," Journal of engineering materials and technology, vol. 119, no. 2, pp. 121-124, 1997.

[44] R. A. Schapery, "Viscoelastic behavior and analysis of composite materials," Mechanics of composite materials.(A 75-24868 10-39) New York, Academic Press, Inc., 1974,, vol. 2.

[45] P. Le Moal and D. Perreux, "Evaluation of creep compliances of unidirectional fibrereinforced composites," Composites science and technology, vol. 51, no. 4, pp. 469477, 1994.

[46] J. Segurado and J. Llorca, "A numerical approximation to the elastic properties of sphere-reinforced composites," Journal of the Mechanics and Physics of Solids, vol. 50, no. 10, pp. 2107-2121, 2002.

[47] D. Peretz and Y. Weitsman, "Nonlinear viscoelastic characterization of fm-73 adhesive," Journal of Rheology, vol. 26, p. 245, 1982.

[48] R. M. Haj-Ali and A. H. Muliana, "Numerical finite element formulation of the schapery non-linear viscoelastic material model," International Journal for Numerical Methods in Engineering, vol. 59, no. 1, pp. 25-45, 2004.

[49] A. Ortiz, R. Bustamante, and K. Rajagopal, "A numerical study of a plate with a hole for a new class of elastic bodies," Acta Mechanica, vol. 223, no. 9, pp. 1971-1981, 2012.

[50] K. Rajagopal and J. Walton, "Modeling fracture in the context of a strain-limiting theory of elasticity: a single anti-plane shear crack," International Journal of Fracture, vol. 169, no. 1, pp. 39-48, 2011. 
[51] R. Rivlin and D. Saunders, "Large elastic deformations of isotropic materials," in Collected Papers of RS Rivlin, pp. 157-194, Springer, 1997. 


\section{APPENDIX}

As we stated in the main body of the dissertation, when the stress is prescribed, the constitutive model defined through Eqn. 4.19 directly gives the corresponding strain. When the strain input is prescribed, it would in general be more challenging to determine the corresponding stress output, and hence, we provide a numerical solution based on a trial solution for the response as a first approximation, which is then followed by an iteration to minimize the error (residual) from the linearizion.

Using a recursive method to solve the integral model 4.19, the time dependent strain is written as:

$$
\begin{aligned}
\varepsilon_{i j}^{t} & =-\alpha\left(B(0)+\Sigma B_{n}\right) f^{t} \sigma_{k k}^{t} \delta_{i j}+\alpha \Sigma B_{n} q_{n}^{t} \delta_{i j} \\
& +\gamma\left(J(0)+\Sigma J_{n}\right) f^{t} \sigma_{i j}^{t}-\gamma \Sigma J_{n} p_{i j, n}^{t},
\end{aligned}
$$

where $q_{n}^{t}$ and $p_{i j, n}^{t}$ are the history variables that incorporates the loading histories and need to be evaluated at each time increment. The history variables at current time is approximated as:

$$
\begin{aligned}
\varepsilon_{i j}^{t} & =-\left[\alpha B(0) f^{t} \sigma_{k k}^{t}-\alpha \Sigma B_{n} f^{t} \sigma_{k k}^{t}+\alpha \Sigma B_{n} \int_{0}^{t} \exp \left[-(t-s) / \tau_{n}\right] \frac{d\left(f_{1} \sigma_{k k}\right)}{d s} d s\right] \\
& \delta_{i j}+\gamma J(0) f^{t} \sigma_{i j}^{t}+\gamma \Sigma J_{n} f^{t} \sigma_{i j}^{t}-\gamma \Sigma J_{n} \int_{0}^{t} \exp \left[-(t-s) / \lambda_{n}\right] \frac{d\left(f_{1} \sigma_{i j}\right)}{d s} d s \\
& =-\alpha\left(B(0)+\Sigma B_{n}\right) f^{t} \sigma_{k k}^{t} \delta_{i j}+\alpha \Sigma B_{n} q_{n}^{t} \delta_{i j} \\
& +\gamma\left(J(0)+\Sigma J_{n}\right) f^{t} \sigma_{i j}^{t}-\gamma \Sigma J_{n} p_{i j, n}^{t}
\end{aligned}
$$


where $q_{n}^{t}$ and $p_{i j, n}^{t}$ are state variables taking the following forms:

$$
\begin{aligned}
q_{n}^{t} & =\int_{0}^{t-\Delta t} \exp \left[-(t-s) / \tau_{n}\right] \frac{d\left(f_{1} \sigma_{k k}\right)}{d s} d s+\int_{t-\Delta t}^{t} \exp \left[-(t-s) / \tau_{n}\right] \frac{d\left(f_{1} \sigma_{k k}\right)}{d s} d s \\
& \approx e^{-\Delta t / \tau_{n}} q_{n}^{t-\Delta t}+\frac{\Delta t}{2}\left[\frac{f_{1}^{t} \sigma_{k k}^{t}-f_{1}^{t-\Delta t} \sigma_{k k}^{t-\Delta t}}{\Delta t}+\left.e^{-\Delta t / \tau_{n}} \frac{\Delta\left(f_{1} \sigma_{k k}\right)}{\Delta t}\right|_{t-\Delta t}\right] \\
p_{i j, n}^{t} & =\int_{0}^{t-\Delta t} \exp \left[-(t-s) / \lambda_{n}\right] \frac{d\left(f_{2} \sigma_{i j}\right)}{d s} d s+\int_{t-\Delta t}^{t} \exp \left[-(t-s) / \lambda_{n}\right] \frac{d\left(f_{2} \sigma_{i j}\right)}{d s} d s \\
& \approx e^{-\Delta t / \lambda_{n}} p_{i j, n}^{t-\Delta t}+\frac{\Delta t}{2}\left[\frac{f_{2}^{t} \sigma_{i j}^{t}-f_{2}^{t-\Delta t} \sigma_{i j}^{t-\Delta t}}{\Delta t}+\left.e^{-\Delta t / \lambda_{n}} \frac{\Delta\left(f_{2} \sigma_{i j}\right)}{\Delta t}\right|_{t-\Delta t}\right]
\end{aligned}
$$

At the initial time $t=0$, the history variables $q_{n}^{t}$ and $p_{i j, n}^{t}$ would be zero and the initial strain $\varepsilon(0)$ will correspond to an elastic response:

$$
\boldsymbol{\varepsilon}(0)=-\alpha B(0) f_{1}(\boldsymbol{\sigma}(0)) \mathbf{I}+\gamma J(0) f_{2}(\boldsymbol{\sigma}(0)) \boldsymbol{\sigma}(0),
$$

The current strain can be expressed in terms of the current stress and history variables after detailed calculations:

$$
\varepsilon_{i j}^{t}=-\bar{B}^{t} \sigma_{k k}^{t} \delta_{i j}+B_{f}^{t} \delta_{i j}+\bar{J}^{t} \sigma_{i j}^{t}-J_{i j, f}^{t},
$$

where

$$
\begin{aligned}
\bar{B}^{t} & =\alpha\left[B(0)+\frac{1}{2} \Sigma B_{n}\right] f_{1}^{t} \\
\bar{J}^{t} & =\gamma\left[J(0)+\frac{1}{2} \Sigma J_{n}\right] f_{2}^{t} \\
B_{f}^{t} & =\alpha \Sigma B_{n}\left[q_{n}^{t}-\frac{1}{2} f_{1}^{t} \sigma_{k k}^{t}\right] \\
J_{i j, f}^{t} & =\gamma \Sigma J_{n}\left[p_{i j, n}^{t}-\frac{1}{2} f_{2}^{t} \sigma_{i j}^{t}\right]
\end{aligned}
$$


Following that, the incremental strain $\Delta \varepsilon_{i j}^{t}$ is defined by

$$
\begin{aligned}
\Delta \varepsilon_{i j}^{t} & =-\left(\bar{B}^{t} \sigma_{k k}^{t}-\bar{B}^{t-\Delta t} \sigma_{k k}^{t-\Delta t}\right) \delta_{i j}+\left(B_{f}^{t}-B_{f}^{t-\Delta t}\right) \delta_{i j} \\
& +\left(\bar{J}^{t} \sigma_{i j}^{t}-\bar{J}^{t-\Delta t} \sigma_{i j}^{t-\Delta t}\right)-\left(J_{i j, f}^{t}-J_{i j, f}^{t-\Delta t}\right)
\end{aligned}
$$

The above equation can be used to determine the unknown stress increment for a given strain increment and the previous history state, $q_{n}^{t}$ and $p_{i j, n}^{t}$. After that, a iterative method is needed in order to arrive the correct stress states. Equation 7 is further linearized after assuming:

$$
\begin{gathered}
\bar{B}^{t}=\bar{B}^{t-\Delta t} \\
\bar{J}^{t}=\bar{J}^{t-\Delta t}
\end{gathered}
$$

After some algebraic manipulations, the approximate incremental stresses can be expressed as

$$
\begin{gathered}
\Delta \sigma_{i j}^{t, t r}=\frac{1}{\bar{J}^{t}}\left(\Delta \varepsilon_{i j}^{t}+J_{i j, f}^{t}-\left(J_{i j, f}^{t}-J_{i j, f}^{t-\Delta t}\right)-\left(B_{f}^{t}-B_{f}^{t-\Delta t}\right) \delta_{i j}\right) \\
+\frac{\bar{B}^{t}}{\left(\bar{J}^{t}-3 \bar{B}^{t}\right) \bar{J}^{t}}\left(\Delta \varepsilon_{k k}^{t}+\left(J_{k k, f}^{t}-J_{k k, f}^{t-\Delta t}\right)-3\left(B_{f}^{t}-B_{f}^{t-\Delta t}\right)\right) \delta_{i j}
\end{gathered}
$$

An iteration scheme is developed in order to find the correct stress states for a given strain increment. The corresponding stress output is obtained by minimizing the residual 
vector $\mathbf{R}$, which is expressed as

$$
\begin{aligned}
R_{i j}^{t} & =-C_{1}\left(f_{1}^{t} \sigma_{k k}^{t}-f_{1}^{t-\Delta t} \sigma_{k k}^{t-\Delta t}\right) \delta_{i j}+\alpha \Sigma B_{n}\left[\left(e^{-\Delta t / \tau_{n}}-1\right) q_{n}^{t-\Delta t}\right. \\
& \left.+\left.\frac{\Delta t}{2} e^{-\Delta t / \tau_{n}} \frac{\Delta\left(f_{1} \sigma_{k k}\right)}{\Delta t}\right|_{t-\Delta t}\right] \delta_{i j} \\
& +C_{2}\left(f_{2}^{t} \sigma_{i j}^{t}-f_{2}^{t-\Delta t} \sigma_{i j}^{t-\Delta t}\right)-\gamma \Sigma J_{n}\left[\left(e^{-\Delta t / \lambda_{n}}-1\right) p_{i j, n}^{t-\Delta t}\right. \\
& \left.+\left.\frac{\Delta t}{2} e^{-\Delta t / \lambda_{n}} \frac{\Delta\left(f_{2} \sigma_{i j}\right)}{\Delta t}\right|_{t-\Delta t}\right]-\Delta \varepsilon_{i j}^{t},
\end{aligned}
$$

where

$$
\begin{aligned}
C_{1} & =\alpha\left[B(0)+\frac{1}{2} \Sigma B_{n}\right] \\
C_{2} & =\gamma\left[J(0)+\frac{1}{2} \Sigma J_{n}\right]
\end{aligned}
$$

Stress at current time is corrected as:

$$
\sigma_{i j}^{t,(k+1)}=\sigma_{i j}^{t,(k)}-\left[\frac{\partial R_{i j}^{t,(k)}}{\partial \sigma_{k l}}\right]^{-1} R_{i j}^{t,(k)},
$$

where $k$ is the iteration counter.

The consistent tangent matrix is defined by

$$
\begin{aligned}
\frac{\partial R_{i j}^{t,(k)}}{\partial \Delta \sigma_{k l}^{t}} & =-C_{1} \frac{\partial f_{1}}{\partial \Delta \sigma_{k l}^{t}} \sigma_{m m}^{t} \delta_{i j}-C_{1} f_{1} \frac{\partial \sigma_{m m}^{t}}{\partial \Delta \sigma_{k l}^{t}} \delta_{i j} \\
& +C_{2} \frac{\partial f_{2}}{\partial \Delta \sigma_{k l}^{t}} \sigma_{i j}^{t}+C_{2} f_{2} \frac{\partial \sigma_{i j}^{t}}{\partial \Delta \sigma_{k l}^{t}}
\end{aligned}
$$

An updated trial stress can be solved from a system of linear equations and is used iteratively until a satisfied level of residual norm can be tolerated, i.e., $\|\mathbf{R}\| \leqslant T o l$. When the converged stress states have been achieved, it is also necessary to update history variables $q_{n}^{t}$ and $p_{i j, n}^{t}$ in Eqn. 3 and calculate $\frac{\Delta\left(f_{1} \sigma_{k k}\right)}{\Delta t}=\frac{f_{1}\left(\sigma_{i j}^{t}\right) \sigma_{k k}^{t}-f_{1}\left(\sigma_{i j}^{t-\Delta t}\right) \sigma_{k k}^{t-\Delta t}}{\Delta t}$ and 
$\frac{\Delta\left(f_{2} \sigma_{i j}\right)}{\Delta t}=\frac{f_{2}\left(\sigma_{i j}^{t}\right) \sigma_{i j}^{t}-f_{2}\left(\sigma_{i j}^{t-\Delta t}\right) \sigma_{i j}^{t-\Delta t}}{\Delta t}$ which are also stored as history variables which will be used in Eqn. 3 and Eqn. 10 in order to continue iteration in the next time integration step. 